\title{
JACOBI-BERNOULLI COHOMOLOGY AND DEFORMATIONS OF SCHEMES AND MAPS
}

\begin{abstract}
ZIV RAN
ABSTRACT. We introduce a notion of Jacobi-Bernoulli cohomology associated to a semi-simplicial Lie algebra (SELA). For an algebraic scheme $X$ over $\mathbb{C}$, we construct a tangent SELA $\mathcal{T}_{X}$ and show that the Jacobi-Bernoulli cohomology of $\mathcal{T}_{X}$ is related to infinitesimal deformations of $X$.
\end{abstract}

\section{O. OVERVIEW}

The 'usual' deformation theory, e.g. of complex structures, in the manner of Kodaira-Spencer-Grothendieck (cf. e.g. [5, 12] and references therein), is commonly couched in terms of a differential graded Lie algebra or dgla $\mathfrak{g}$. It can be viewed, as in [10], as studying the canonically defined deformation ring $R(\mathfrak{g})$, fashioned from the Jacobi cohomology, i.e. the cohomology of the Jacobi complex associated to $\mathfrak{g}$. This setting is somewhat restrictive, e.g. it is not broad enough to accomodate such naturally occurring deformation problems as embedded deformations of a submanifold $X$ in a fixed ambient space $Y$. In [11] we introduced the notion of Lie atom (essentially, Lie pair) and an associated Jacobi-Bernoulli complex as an extension of that of dgla and its Jacobi complex, one that is broad enough to handle embedded deformations and a number of other problems besides.

A purpose of this paper is to establish the familiar notion of (dg) semi-simplicial Lie algebra (SELA) as an appropriately general and convenient setting for deformation theory. As a first approximation, one can think of SELA as a structure like that of the Čech complex of a sheaf of Lie algebras on a topological space $X$ with respect to some open covering of $X$. Not only is SELA a broad generalization of Lie atom, it is broad enough, as we show, to encompass deformations of (arbitrarily singular) algebraic schemes (over $\mathbb{C}$ ).

To compute the deformation theory of a SELA g. we introduce a complex that we call the Jacobi-Bernoulli complex of $\mathfrak{g}_{\bullet}$, though a more proper attribution would be to Jacobi-Bernoulli-Baker-Campbell-Hausdorff. In a nutshell, the

1991 Mathematics Subject Classification. 14D15, 58H15.

Key words and phrases. deformations, schemes, Lie algebras, Bernoulli numbers, cohomology.

Research supported in part by NSA grant H98230-05-1-0063 and by the Mittag-Leffler Institute 06-07 Moduli Spaces program. 
point of this complex is that it transforms a gluing condition from nonabelian coycle condition to ordinary (additive) cocycle condition via the multilinearity of the groups making up the complex. A typical gluing condition looks like

$$
\Psi_{\alpha \beta} \Psi_{\beta \gamma} \Psi_{\gamma \alpha}=1
$$

with $\Psi_{\alpha \beta} \in \exp \left(\mathfrak{g}_{\alpha \beta}\right)$, where $\mathfrak{g}_{\alpha \beta}$ may be thought of as the component of our SELA g. having to do with gluing over $U_{\alpha} \cap U_{\beta}$. This condition can be transformed as follows. Write

$$
\Psi_{\alpha \beta}=\exp \left(\psi_{\alpha \beta}\right)
$$

etc. Now the $\mathrm{BCH}$ formula gives a formal expression

$$
\exp (X) \exp (Y) \exp (Z)=\sum W_{i, j, k}(X, Y, Z)
$$

where $W_{i, j, k}(X, Y, Z)$ is a homogeneous ad-polynomial of tridegree $i, j, k$ ('BCH polynomial'), which can be viewed as a linear map

$$
w_{i j k}: \operatorname{Sym}^{i}\left(\mathfrak{g}_{\alpha \beta}\right) \otimes \operatorname{Sym}^{j}\left(\mathfrak{g}_{\beta \gamma}\right) \otimes \operatorname{Sym}^{k}\left(\mathfrak{g}_{\gamma \alpha}\right) \rightarrow g_{\alpha \beta \gamma}
$$

Then the above gluing condition on the $\Psi$. becomes the additive condition that

$$
w_{i, j, k}\left(\psi_{\alpha \beta}^{i} \otimes \psi_{\beta \gamma}^{j} \otimes \psi_{\gamma \alpha}^{k}\right)=0, \forall i, j, k .
$$

Now our Jacobi-Bernoulli complex $J\left(\mathfrak{g}_{\bullet}\right)$ for the SELA g. is essentially designed so as to encompass the various $\mathrm{BCH}$ polynomials $w_{i j k}$. It is a comultiplicative complex whose groups essentially constitute the symmetric algebra on $\mathfrak{g}_{\bullet}$ and whose maps are essentially derived from the $w_{i j k}$ by the requirement of comultiplicativity. The dual of the cohomology of $J\left(\mathfrak{g}_{\bullet}\right)$ yields the deformation ring associated to the SELA g. .

As mentioned above, our other main result here is that the deformation theory of an algebraic scheme over $\mathbb{C}$ can be expressed in terms of a SELA . Unsurprisingly, this is done via an affine covering. Thus the first step is to associate a dgla to a closed embedding

$$
X \rightarrow P
$$

where $P$ is an affine (or for that matter, projective) space. We call this the tangent dgla to $X$ and denote it $\mathcal{T}_{X}(P)$. In a nutshell, $\mathcal{T}_{X}(P)$ is defined as the mapping cone of a map that we construct

$$
T_{P} \otimes A_{X} \rightarrow N_{X / P}
$$

where $N_{X / P}$ is the normal atom to $X$ in $P$ as in [11]. That is, $\mathcal{T}_{X}(P)$ is represented by the mapping cone of a map of free modules representing $T_{P} \otimes A_{X}$ and $N_{X / P}$. We will show $\mathcal{T}_{X}(P)$ admits a dgla structure, a dgla action on $A_{X}$, as well as $A_{X}$-module structure. Up to a certain type of 'weak equivalence', the dgla $\mathcal{T}_{X}(P)$ depends only on the isomorphism class of $X$ and not on the embedding in $P$.

The partial independence on the embedding is good enough to enable us to associate a global SELA $\mathcal{T}_{X}$. for an arbitrary algebraic scheme $X$ defined in terms 
of, but up to weak equivalence independent of, an affine covering $X_{\alpha}$ and embeddings of each $X_{\alpha}$ in an affine space $P_{\alpha}$ : e.g.

$$
\begin{gathered}
\mathcal{T}_{X, \alpha}=\mathcal{T}_{X_{\alpha}}\left(P_{\alpha}\right), \\
\mathcal{T}_{\mathcal{X}, \alpha \beta}=\mathcal{T}_{\mathcal{X}_{\alpha} \cap X_{\beta}}\left(P_{\alpha} \times P_{\beta}\right)
\end{gathered}
$$

etc. Global deformations of $X$ then amount to a collections of deformations of each $X_{\alpha}$, given via Kodaira-Spencer theory by a suitable element $\phi_{\alpha} \in \mathcal{T}_{X, \alpha}^{1}$, plus a collection of gluing data $\psi_{\alpha \beta} \in \mathcal{T}_{X, \alpha \beta}^{0}$, and the necessary compatibilities are readily expressed as a cocycle condition in the Jacobi-Bernoulli complex $J\left(\mathcal{T}_{X} \bullet\right)$.

Remark 0.1. At the time of writing (Sep '07), this preprint is not yet in final form; in particular, it has portions developed at different times which have yet to be fully synchronized. Comments or questions from readers would be most welcome.

\section{Semi-Simplicial Lie algebras and Jacobi-Bernoulli complex}

1.1. SELA. Our notion of SELA is essentially the dual of the portion of the usual notion of simplicial Lie algebra involving only the face maps without degeneracy maps. Let $A$ be a totally ordered index-set. A simplex in $A$ is a finite nonempty subset $S \subset A$, while a biplex is a pair $\left(S_{1} \subset S_{2}\right)$ of simplices with $\left|S_{1}\right|+1=\left|S_{2}\right|$; similarly for triplex $\left(S_{1} \subset S_{2} \subset S_{3}\right)$ etc. The sign $\epsilon\left(S_{1}, S_{2}\right)$ of a biplex $\left(S_{1}, S_{2}\right)$ is defined by the condition that

$$
\epsilon((0, \ldots, \hat{p}, \ldots, n),(0, \ldots, n))=(-1)^{n-p} .
$$

By a semi simplicial Lie algebra (SELA) $\mathfrak{g}_{\bullet}$ on $A$ we shall mean the assignment for each simplex $S$ on $A$ of a Lie algebra $\mathfrak{g}_{S}$, and for each biplex $\left(S_{1}, S_{2}\right)$ of a map ('coface' or 'restriction')

$$
r_{S_{1}, S_{2}}: \mathfrak{g}_{S_{1}} \rightarrow \mathfrak{g}_{S_{2}}
$$

such that $\epsilon\left(S_{1}, S_{2}\right) r\left(S_{1}, S_{2}\right)$ is a Lie homomorphism and such that for each $S_{1} \subset S_{3}$ with $\left|S_{1}\right|+2=\left|S_{3}\right|$, we have

$$
\sum_{\substack{\text { triplex } \\\left(S_{1} \subset S_{2} \subset S_{3}\right)}} r_{\left(S_{2}, S_{3}\right)} r_{\left(S_{1}, S_{2}\right)}=0 .
$$

The identity (1. 1.1) implies that we may assemble the $\mathfrak{g}_{S}$ into a complex $K \cdot\left(\mathfrak{g}_{\bullet}\right)$ where

$$
K^{i}\left(\mathfrak{g}_{\bullet}\right)=\bigoplus_{|S|=i+1} \mathfrak{g}_{S}
$$

and differential constructed from the various $r_{\left(S_{1}, S_{2}\right)}$. 
Example 1.1. If $\mathfrak{g}$ is a sheaf of Lie algebras on a topological space $X$, and $\left(U_{\alpha}\right)$ is an open covering of $X$, there is a Čech SELA

$$
\mathfrak{g}_{\bullet}: S \mapsto \mathfrak{g}\left(\bigcap_{\alpha \in S} U_{\alpha}\right) \text {. }
$$

The standard complex $K^{\cdot} \cdot\left(\mathfrak{g}_{\bullet}\right)$ is the Čech complex $\check{C}\left(\mathfrak{g},\left(U_{\alpha}\right)\right)$; it plays a fundamental role in the study of $\mathfrak{g}$-deformations.

Example 1.2. If $\mathfrak{g} \rightarrow \mathfrak{h}$ is a Lie pair (more generally, a Lie atom, cf. [11]), we get a SELA $\mathfrak{g}$. on the index-set (01) with $\mathfrak{g}_{0}=\mathfrak{g}, \mathfrak{g}_{1}=0, \mathfrak{g}_{01}=\mathfrak{h}$.

$$
\mathfrak{g} \bullet \stackrel{\mathfrak{h}}{\longrightarrow} 0
$$

The deformation-theoretic significance of $\mathfrak{g}$. is like that of the Lie atom $(\mathfrak{g}, \mathfrak{h})$, viz. $\mathfrak{g}$-deformations together with an $\mathfrak{h}$-trivialization.

An obvious generalization would be to take a pair of maps $\mathfrak{g}_{1} \rightarrow \mathfrak{h}, \mathfrak{g}_{2} \rightarrow \mathfrak{h}$ (e.g. twice the same map), which corresponds to pairs $\left(\mathfrak{g}_{1}-\right.$ deformation, $\mathfrak{g}_{2}-$ deformation) such that the induced $\mathfrak{h}$-deformations are equivalent as such.

1.2. Bernoulli numbers and Baker-Campbell-Hausdorff. Let $\mathfrak{g}$ be a nilpotent Lie algebra. For an element $X \in \mathfrak{g}$ we consider the formal exponential $\exp (X)$ as an element of the formal enveloping algbera $\mathfrak{U}(\mathfrak{g})$. Then we can write

$$
\exp (X) \exp (Y)=\exp (\beta(X, Y))
$$

where $\beta$ is a certain bracket-polynomial in $X, Y$, known as the Baker-CampbellHausdorff or $\mathrm{BCH}$ polynomial. We denote by $\beta_{i, j}, \beta_{i}$ the portion of $\beta$ in bidegree $i, j$ (resp. total degree $i$ ). Note that each $\beta_{i, j}(X, Y)$ will be a linear combination of (noncommutative) ad monomials with a total of $i$ many $X$ 's and $j$ many $Y$ 's. We write such a monomial in the form

$$
\operatorname{ad}_{S}\left(X^{i} Y^{j}\right)=\operatorname{ad}\left(T_{1}\right) \circ \ldots \circ \operatorname{ad}\left(T_{i+j-1}\right)\left(T_{i+j}\right)
$$

where $S \subset[1, i+j]$ is a subset of cardinality $i$ and $T_{k}=X$ (resp. $T_{k}=Y$ ) iff $k \in S$ (resp. $k \notin S$ ). We denote by

$$
\operatorname{ad}_{S}\left(X_{1}, \ldots, X_{i}, Y_{1}, \ldots, Y_{j}\right)
$$

the analogous function, obtained by replacing the $x$ th occurrence of $X$ (resp. $y$ th occurrence of $Y$ ) by an $X_{x}$ (resp. $Y_{y}$ ) and by $\operatorname{ad}_{S}^{\mathrm{Sym}}\left(X_{1}, \ldots, X_{i}, Y_{1}, \ldots, Y_{j}\right)$ the corresponding symmetrized version, i.e.

$$
\operatorname{ad}_{S}^{\text {Sym }}\left(X_{1}, \ldots, X_{i}, Y_{1}, \ldots, Y_{j}\right)=\sum_{\substack{\pi \in \mathfrak{S}_{i} \\ \rho \in \mathfrak{S}_{j} \\ 4}} \frac{1}{i ! j !} \operatorname{ad}_{S}\left(X_{\pi(1)}, \ldots, X_{\pi(i)}, Y_{\rho(1)}, \ldots, Y_{\rho(j)}\right) .
$$


We will compute $\beta$, following [13], $\S 2.15$ (where Varadarajan attributes the argument to lectures of Bargmann that follow original papers by Baker and Hausdorff). Set

$$
D(x)=\frac{e^{x}-1}{x}, C(x)=1 / D(x) .
$$

Thus, $C(x)$ is the generating function for the Bernoulli numbers $B_{n}$, i.e.

$$
C(x)=1+\sum_{n=1}^{\infty} \frac{B_{n}}{n !} x^{n}=\sum_{n=0}^{\infty} C_{n} x^{n} .
$$

Now the reader can easily check that for any derivation $\partial$ we have

$$
\partial \exp (U) \exp (-U)=D(\operatorname{ad}(U))(\partial U), \exp (-U) \partial \exp (U)=D(-\operatorname{ad}(U))(\partial U) .
$$

Now differentiate (1.2.2) with respect to $X$ and multiply both sides by $\exp \left(-\beta(X, Y)\right.$ ). This yields (where $\partial_{X}$ is the unique derivation taking $X$ to $X$ and $Y$ to 0$)$

$X=\partial_{X}(\exp (X)) \exp (-X)=$

$$
=\partial_{X}(\exp (\beta(X, Y))) \exp (-\beta(X, Y))=D(\operatorname{ad}(\beta(X, Y)))\left(\partial_{X} \beta(X, Y)\right) .
$$

Thus

$$
\partial_{X} \beta(X, Y)=C(\operatorname{ad}(\beta(X, Y)))(X) .
$$

Similarly,

$$
\partial_{Y} \beta(X, Y)=C(-\operatorname{ad}(\beta(X, Y)))(Y) \text {. }
$$

Starting from $\beta_{0}=0$, the formulas (1. 2.7),(1. 2.8) clearly determine $\beta$. For example, clearly $\beta_{0, *}(X, Y)=Y$, therefore it follows that

$$
\beta_{1, *}(X, Y)=C(\operatorname{ad}(Y))(X)=X+\frac{1}{2}[X, Y]+\frac{1}{12} \operatorname{ad}(Y)^{2}(X)+\ldots
$$

We shall require the obvious extension of this set-up to the trivariate case. Thus define a function $\beta(X, Y, Z)$ (NB same letter as for the bivariate version) by

$$
\exp (X) \exp (Y) \exp (Z)=\exp (\beta(X, Y, Z))
$$

and let $\beta_{i, j, k}$ denote its portion in tridegree $(i, j, k)$. Note that

$$
\beta(X, Y, Z)=\beta(\beta(X, Y), Z) .
$$


1.3. Jacobi-Bernoulli complex. Let $\mathfrak{g}$. be a SELA . For simplicity, we shall assume $\mathfrak{g}_{\bullet}$ is 2- dimensional, in the sense that $\mathfrak{g}_{S}=0$ for any simplex $S$ of dimension $>2$; for our applications to deformation theory, this is not a significant restriction. We will also assume that $\mathfrak{g}_{0}$ is strongly nilpotent in the sense that it is an algebra over a commutative ring $R$ such that $\mathfrak{g}_{S}^{\otimes N}=0$ for all simplices $S$ and some integer $N$ independent of $S$, with all tensor products over $R$. This condition obviously depends only on the $S$-module structure of $\mathfrak{g}_{\bullet}$ and not on its Lie bracket. We are going to define a filtered complex $J=J_{\mathrm{m}}^{\sharp}\left(\mathfrak{g}_{\bullet}\right)$. The groups $J^{j}$ can be defined succinctly as

$$
J^{j}=\left(\operatorname{Sym} \cdot\left(K \cdot\left(\mathfrak{g}_{\bullet}\right)[1]\right)\right)^{j}
$$

where Sym is understood in the signed or graded sense, alternating on odd terms, and $K \cdot\left(\mathfrak{g}_{\bullet}\right)[1]$ is the standard complex on $\mathfrak{g}_{\bullet}$ shifted left once (which is a complex in degrees $-1,0,1)$. The increasing filtration $F$. is by "number of multiplicands'. More concretely,

$$
J^{j, k}=\bigoplus_{\substack{-\sum_{i} \ell_{i}+\sum_{i} n_{i}=j \\ \sum_{i} \ell_{i}+\sum_{i} m_{i}+\sum_{i} n_{i}=k}} \bigotimes^{i} \bigwedge^{\ell_{i}} \mathfrak{g}_{\alpha_{i}} \otimes \bigotimes^{i} \operatorname{Sym}^{m_{i}} \mathfrak{g}_{\alpha_{i} \beta_{i}} \otimes \bigotimes^{i} \bigwedge^{n_{i}} \mathfrak{g}_{\alpha_{i} \beta_{i} \gamma_{i}}
$$

$$
F_{m} J^{j}=\bigoplus_{k \leq m} J^{j, k}
$$

$$
J^{j}=F_{\infty} J^{j}=F_{N} J^{j} .
$$

To define the differential $d$ on $J$, we proceed in steps. Let $\alpha<\beta<\gamma$ be indices and recall that we are identifying $\mathfrak{g}_{\gamma \alpha}$ with $\mathfrak{g}_{\alpha \gamma}$.

- The differential is defined so that the obvious inclusion

$$
\left.K \cdot\left(\mathfrak{g}_{\bullet}\right)[1]\right)=F_{1} J \cdot J^{\prime}
$$

is a map of complexes.

- The component

$$
\operatorname{Sym}^{i} \mathfrak{g}_{\gamma \alpha} \otimes \operatorname{Sym}^{j} \mathfrak{g}_{\alpha \beta} \otimes \operatorname{Sym}_{\beta \gamma}^{\mathfrak{g}} \rightarrow \mathfrak{g}_{\alpha \beta \gamma}
$$

is given by

$$
X^{i} Y^{j} Z^{k} \mapsto \beta_{i, j, k}(X, Y, Z) .
$$

- The component

$$
\mathfrak{g}_{\alpha} \otimes \operatorname{Sym}^{i} \mathfrak{g}_{\alpha \beta} \otimes \operatorname{Sym}^{n} \mathfrak{g}_{\beta \gamma} \rightarrow \operatorname{Sym}^{i-t+1} \mathfrak{g}_{\alpha \beta} \otimes \operatorname{Sym}^{n} \mathfrak{g}_{\beta \gamma}, 0 \leq t \leq i
$$

is given by

$$
X \otimes Y^{i} \otimes Z^{n} \mapsto C_{t} Y^{i-t} \operatorname{ad}(Y)^{t}(X) \otimes Z^{n}
$$

(where $C_{t}$ is the normalized Bernoulli coefficient). 
- All componets are defined subject to the 'derivation rule', which means commutativity of the following diagram

$$
\begin{aligned}
& \forall i=i_{1}+i_{2}, j=j_{1}+j_{2}, \quad i_{1}, i_{2}, j_{1}, j_{2}>0:
\end{aligned}
$$

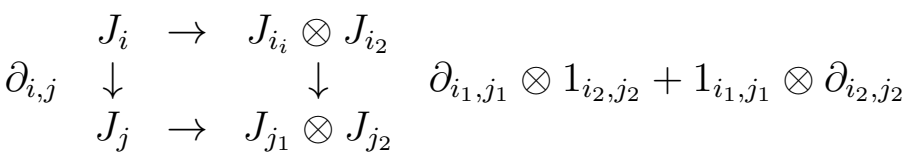

in which $J_{i}=\bigoplus_{j} J^{j, i}$ denotes the group direct summand of $J$ in multiplicative degree $i$ and $\partial_{i, j}$ denotes the component of $\partial$ going from $J_{i}$ to $J_{j}$, and $1_{i, j}$ is the identity if $i=j$ and zero otherwise; and of course the horizontal arrows are the appropriate components of the comultiplication map. E.g. the component

$$
\mathfrak{g}_{\alpha} \otimes \operatorname{Sym}^{i} \mathfrak{g}_{\beta \gamma} \otimes \operatorname{Sym}^{k} \mathfrak{g}_{\beta \gamma} \rightarrow \mathfrak{g}_{\gamma \alpha} \otimes \operatorname{Sym}^{i} \mathfrak{g}_{\alpha \beta} \otimes \operatorname{Sym}^{k} \mathfrak{g}_{\beta \gamma}
$$

is extended in the obvious way from the given differential $\mathfrak{g}_{\alpha} \rightarrow \mathfrak{g}_{\gamma \alpha}$ which comes with the SELA data.

- Components not defined via the above rules are set equal to 0. In particular, the component

is zero.

$$
\mathfrak{g}_{\alpha} \otimes \mathfrak{g}_{\alpha \beta \gamma} \rightarrow \mathfrak{g}_{\alpha \beta \gamma}
$$

The following result summarizes the main properties of the Jacobi-Bernoulli complex $J$ associated to a SELA (not least, that it is a complex!). It is in part, but not entirely, a direct extension of the analogous result for Lie atoms given in [11].

Theorem 1.3. $\quad$ (i) $(J, F$.) is a functor from the category of SELAs over $S$ to that of comultiplicative, cocummutative and coassociative filtered complexes over $S$.

(ii) The filtration $F$. is compatible with the comultiplication and has associated graded

$$
F_{i} / F_{i-1}=\bigwedge^{i} K^{\cdot}\left(\mathfrak{g}_{\mathbf{\bullet}}\right)
$$

(iii) The quasi-isomorphism class of $J\left(\mathfrak{g}_{\bullet}\right)$ depends only on the quasi-isomorphism class of $\mathfrak{g}$. as SELA .

Proof. As in the proof of [11], Thm 1.2.1, the main issue is to prove $J$ is a complex, i.e. $d^{2}=0$. And again as in [11], it suffices, in light of the derivation rule, to prove the vanishing of the components of $d^{2}$ that land in $F_{1}$, i.e. that have just one multiplicative factor. Among those, the proof that these components of $d^{2}$ vanish on terms of degree $\leq-2$, i.e. involving $\bigwedge^{i} \mathfrak{g}_{\alpha}, i \geq 2$, is similar to the case of the JB complex considered in [11]. The essential new case, not considered in [11], is 
the vanishing of the $F_{1}$ - components of $d^{2}$ on terms of degree -1, i.e. terms of the from

$$
X \otimes Y^{i} \otimes Z^{n} \in \mathfrak{g}_{\alpha} \otimes \operatorname{Sym}^{i} \mathfrak{g}_{\alpha \beta} \otimes \operatorname{Sym}^{n} \mathfrak{g}_{\beta \gamma} .
$$

For such a term, what needs to be shown is the vanishing of the component of $d^{2}$ of it in $\mathfrak{g}_{\alpha \beta \gamma}$. Thus, we need to prove that

$$
d^{2}\left(X \otimes Y^{i} \otimes Z^{n}\right)_{\mathfrak{g}_{\alpha \beta \gamma}}=0 .
$$

Now this component gets contributions via the various components of $d\left(X \otimes Y^{i} \otimes\right.$ $\left.Z^{n}\right)$ and those contributions come in two kinds:

- Via $g_{\gamma \alpha} \otimes \operatorname{Sym}^{i} \mathfrak{g}_{\alpha \beta} \otimes \operatorname{Sym}^{n} \mathfrak{g}_{\beta \gamma}$, we get $-\beta_{1, i, n}(X, Y, Z)$. This comes from $\beta(\beta(X, Y), Z)$, but is only affected by the terms in $\beta(X, Y)$ of degree $\leq 1$ in $X$, i.e. by

$$
U=Y+C(\operatorname{ad}(Y))(X)=Y+\sum_{t=0}^{\infty} C_{t} \operatorname{ad}(Y)^{t}(X) .
$$

This contribution is obtained by taking $-\beta_{i+1-t, n}(U, Z)$ and replacing each monomial

$$
\operatorname{ad}_{S}\left(U^{i+1-t} Z^{n}\right)
$$

(cf. (1. 2.3-1.2.5) ) by

$$
(i+1-t) \operatorname{ad}_{S}^{\text {Sym }}\left(C_{t}\left(\operatorname{ad}(Y)^{t}(X)\right) Y^{i-t} Z^{n}\right)
$$

where and finally summing as $t$ ranges from 0 to $i$.

- Via $\operatorname{Sym}^{i+1-t} \mathfrak{g}_{\alpha \beta} \otimes \operatorname{Sym}^{n} \mathfrak{g}_{\beta \gamma}$, for each $0 \leq t \leq i$, we get a contribution equal to the expression obtained by taking $\beta_{i+1-t, n}(W, Z)$, replacing each monomial

$$
\operatorname{ad}_{S}\left(W^{i+1-t} Z^{n}\right)
$$

by

$$
(i+1-t) \operatorname{ad}_{S}^{\text {Sym }}\left(C_{t} \operatorname{ad}(Y)^{t}(X) Y^{i-t} Z^{n}\right) .
$$

Thus, the sum total of all contributions to $d^{2}\left(X \otimes Y^{i} \otimes Z^{n}\right)_{\mathfrak{g}_{\alpha \beta \gamma}}$ is zero.

The local ring

$$
\left(R\left(\mathfrak{g}_{\bullet}\right), \mathfrak{m}_{R\left(\mathfrak{g}_{\bullet}\right)}\right)=\left(\mathbb{C} \oplus \mathbb{H}^{0}\left(J\left(\mathfrak{g}_{\bullet}\right)\right)^{*}, \mathbb{H}^{0}\left(J\left(\mathfrak{g}_{\bullet}\right)\right)^{*}\right)
$$

is called the deformation ring of $\mathfrak{g}_{\bullet}$. Its (more meaningful) $\mathfrak{m}$-adic completion is denoted $\hat{R}\left(\mathfrak{g}_{\bullet}\right)$ and called the formal deformation ring of $\mathfrak{g}_{\mathbf{0}}$. The formal spectrum $\operatorname{Spf}\left(\hat{R}\left(\mathfrak{g}_{\bullet}\right)\right.$ is called the formal deformation space of $\mathfrak{g}_{\bullet}$ and denoted $\operatorname{Deff}\left(\mathfrak{g}_{\bullet}\right)$.

Corollary 1.4. Assume that $K^{\cdot}\left(\mathfrak{g}_{\bullet}\right)$ is acyclic in nonpositive degrees. Then there is a second-quadrant spectral sequence with $E_{1}$ term

$$
E_{1}^{p, q}=\operatorname{Sym}_{8}^{-q-2 p} \mathcal{H}^{1}\left(\mathfrak{g}_{\bullet}\right) \otimes \operatorname{Sym}^{q+p} \mathcal{H}^{2}\left(\mathfrak{g}_{\bullet}\right)
$$


whose abutment has degree 0 part $\bigoplus E_{\infty}^{p,-p}$ equalt to

$$
\text { (1. 3.20) } \quad E_{\infty}^{p,-p}=\bigoplus g r_{F}^{-p} \mathcal{H}^{0}\left(J\left(\mathfrak{g}_{\bullet}\right)\right) \text {. }
$$

Proof. Identical to that of [11], Cor. 1.2.2.

Corollary 1.5. If $\mathfrak{g}_{\mathbf{0}}$ is acyclic in nonpositive degrees and $h^{1}\left(\mathfrak{g}_{\bullet}\right)$ and $h^{2}\left(\mathfrak{g}_{\mathbf{\bullet}}\right)$ are finite, then we have a lower bound for the Krull dimension of its formal deformation ring:

$$
\operatorname{dim} \hat{R}\left(\mathfrak{g}_{\bullet}\right) \geq h^{1}\left(\mathfrak{g}_{\bullet}\right)-h^{2}\left(\mathfrak{g}_{\bullet}\right) .
$$

Proof. Cf. [11], Cor. 1.2.3.

1.4. Special multiplicative cocycles. Let $\left(S, \mathfrak{m}_{S}\right)$ be a local artin $\mathbb{C}$-algebra and let $\mathfrak{g}_{\mathbf{0}}=\mathfrak{g}_{\bullet}$ be a dg-SELA (i.e. for each simplex $T, \mathfrak{g}_{T}$ is a dgla and the coface maps are $\pm \mathrm{dg}$ homomorphisms). A special class of (multiplicative) cocycles for the Jacobi-Bernoulli complex

$$
J \cdot\left(\mathfrak{g}_{\bullet} \otimes \mathfrak{m}_{S}\right) \subset J \cdot\left(\mathfrak{g}_{\bullet}\right) \otimes \mathfrak{m}_{S}
$$

can be constructed as follows. Suppose

$$
\begin{aligned}
& \phi_{\bullet} \in K^{0}\left(\mathfrak{g}_{\bullet}\right)^{1} \otimes \mathfrak{m}_{S}=\bigoplus_{\rho} \mathfrak{g}_{\rho}^{1} \otimes \mathfrak{m}_{S}, \\
& \psi_{\bullet} \in K^{1}\left(\mathfrak{g}_{\bullet}\right)^{0} \otimes \mathfrak{m}_{S}=\bigoplus_{\rho \sigma} \mathfrak{g}_{\rho \sigma}^{0} \otimes \mathfrak{m}_{S}
\end{aligned}
$$

are such that, $\forall \rho, \sigma, \tau$,

$$
\begin{aligned}
\partial \phi_{\rho} & =-\frac{1}{2}\left[\phi_{\rho}, \phi_{\rho}\right], \\
\partial \psi_{\rho \sigma} & =-C\left(\operatorname{ad}\left(\psi_{\rho \sigma}\right)\right)\left(\phi_{\rho}\right)+C\left(-\operatorname{ad}\left(\psi_{\rho \sigma}\right)\right)\left(\phi_{\sigma}\right), \\
\beta\left(\psi_{\rho \sigma}, \psi_{\sigma \tau}, \psi_{\tau \rho}\right) & =0 .
\end{aligned}
$$

Then let

$$
\epsilon\left(\phi_{\bullet}, \psi_{\bullet}\right) \in J^{0}\left(\mathfrak{g}_{\bullet}\right) \otimes \mathfrak{m}_{S}
$$

be the element with components

$$
\begin{gathered}
\psi_{\rho \sigma} \in \mathfrak{g}_{\rho \sigma} \otimes \mathfrak{m}_{S}, \\
\phi_{\rho} \in \mathfrak{g}_{\rho} \otimes \mathfrak{m}_{S},
\end{gathered}
$$

and generally

$$
\bigwedge^{r} \phi_{\rho} \otimes \bigotimes\left(\psi_{\rho_{i} \sigma_{i}}\right)^{n_{i}} \in \bigwedge^{r}\left(\mathfrak{g}_{\rho}^{1} \otimes \mathfrak{m}_{S}\right) \otimes \bigotimes \operatorname{Sym}^{n_{i}}\left(\mathfrak{g}_{\rho_{i} \sigma_{i}} \otimes \mathfrak{m}_{S}\right), r, n \geq 0 .
$$

We call $\epsilon\left(\phi_{\bullet}, \phi_{\bullet}\right)$ a special multiplicative cocycle with coefficients in $S$. 
Lemma 1.6. (i) Assuming (1.4.21-1. 4.23), the cochain $\epsilon\left(\phi_{\bullet}, \psi_{\bullet}\right)$ defined in (1. 4.24) is a 0 -cocycle for $J\left(\mathfrak{g}_{\bullet} \otimes \mathfrak{m}_{S}\right)$ and the associated map

$$
{ }^{t}\left[\epsilon\left(\phi_{\bullet}, \psi_{\bullet}\right)\right] \in \operatorname{Hom}\left(R\left(\mathfrak{g}_{\bullet}\right), S\right)
$$

is a local ring homomorphism.

(ii) Assume $K \cdot\left(\mathfrak{g}_{\bullet}\right)$ is acyclic in negative degrees. Then given an artin local algebra $S$, there is a natural bijection between the set of cohomology classes

$$
\left[\epsilon\left(\phi_{\bullet}, \psi_{\bullet}\right)\right] \in H^{0}\left(J \cdot\left(\mathfrak{g}_{\bullet} \otimes \mathfrak{m}_{S}\right)\right)
$$

of special multiplicative cocycles with coefficients in $S$ and the set of local ring homomorphisms $R\left(\mathfrak{g}_{\mathbf{0}}\right) \rightarrow S$.

Proof. (i) Because of the derivation property of the differentials in the complex $J$, it will suffice to prove the vanishing of the portion of the differential $\partial \epsilon\left(\phi_{\bullet}, \psi_{\bullet}\right)$ in $F_{1} J$ (i.e. having a single tensor factor). That portion had just three components: in $K^{0}\left(\mathfrak{g}_{\bullet}\right) \otimes \mathfrak{m}_{S}, K^{1}\left(\mathfrak{g}_{\bullet}\right)^{1}$ and $K^{2}\left(\mathfrak{g}_{\bullet}\right)^{0}$, and the vanishing of these yields, respectively eq. (1. 4.21-1. 4.23).

Now given that $\epsilon$ is a cocycle, the fact that ${ }^{t}[\epsilon]$ is multiplicative follows from the fact, clear by construction, that $\epsilon=\epsilon\left(\phi_{\bullet}, \psi_{\bullet}\right)$ is compatible with the comultiplication on $J \cdot=J \cdot\left(\mathfrak{g}_{\bullet} \otimes \mathfrak{m}_{S}\right)$, in the sense that the image of $\epsilon$ in $J \cdot \otimes J \cdot$ is just $\epsilon \otimes \epsilon$.

(ii) Note that because $K \cdot\left(\mathfrak{g}_{\bullet}\right)$ is acyclic in negative degrees, the natural map

$$
H^{0}\left(J \cdot\left(\mathfrak{g}_{\bullet} \otimes \mathfrak{m}_{S}\right)\right) \rightarrow H^{0}\left(J \cdot\left(\mathfrak{g}_{\bullet}\right)\right) \otimes \mathfrak{m}_{S}
$$

is injective, so any class $\gamma \in H^{0}\left(J \cdot\left(\mathfrak{g}_{\bullet} \otimes \mathfrak{m}_{S}\right)\right)$ is determined by the associated map ${ }^{t} \gamma: R\left(\mathfrak{g}_{\mathfrak{b}}\right) \rightarrow S$. Hence, our assertion is a matter of showing that for any local $\mathbb{C}$-algebra homomorphism $f: R\left(\mathfrak{g}_{\bullet}\right) \rightarrow S$, the corresponding cohomology class $\eta \in H^{0}\left(J \cdot\left(\mathfrak{g}_{\bullet}\right)\right) \otimes \mathfrak{m}_{S}$ can be represented in the form $\left[\epsilon\left(\phi_{\bullet}, \psi_{\bullet}\right)\right]$, where

$$
\left(\phi_{\bullet}, \psi_{\bullet}\right) \in\left(K^{0}\left(\mathfrak{g}_{\bullet}\right)^{1} \otimes K^{1}\left(\mathfrak{g}_{\bullet}\right)^{0}\right) \otimes \mathfrak{m}_{S}
$$

is just the component of (a representative of) $\eta$ of multiplicative degree 1, i.e. in $F_{1}(J)$, where $J=J \cdot\left(\mathfrak{g}_{\bullet}\right) \otimes \mathfrak{m}_{S}$. We may assume $f: R_{k}\left(\mathfrak{g}_{\bullet}\right) \rightarrow S$ where $k$ is the exponent of $S$ and, by induction, that the result holds for $S$ of exponent $<k$. Set $\epsilon=\epsilon\left(\phi_{\bullet}, \psi_{\bullet}\right)$. By induction, $\epsilon$ is a cocycle modulo the socle $I=\operatorname{ann}_{S}\left(\mathfrak{m}_{S}\right)$ of $S$ and $\eta \equiv[\epsilon] \bmod I$. Moreover, because $\mathfrak{m}_{R_{k}\left(\mathfrak{g}_{\bullet}\right)}^{2}$ and $\mathfrak{m}_{S}^{2}$ are quotients, respectively, of $\mathfrak{m}_{R_{k-1}\left(\mathfrak{g}_{\bullet}\right)}$ and $\mathfrak{m}_{S / I}$, the images of $\eta$ and $[\epsilon]$ in $\left(J / F_{1}(J)\right) \otimes \mathfrak{m}_{S}$ (whic are automatically in $\left(J / F_{1}(J)\right) \otimes \mathfrak{m}_{S}^{2}$ also coincide. It follows that there is a cochain

$$
\left(\phi_{\bullet}^{\prime}, \psi_{\bullet}^{\prime}\right) \in\left(K^{0}\left(\mathfrak{g}_{\bullet}\right)^{1} \otimes K^{1}\left(\mathfrak{g}_{\bullet}\right)^{0}\right) \otimes I
$$

such that

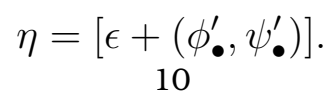


However, since $\left(\phi_{\bullet}^{\prime}, \psi_{\bullet}^{\prime}\right)$ has coefficients in the socle while $\epsilon$ has coefficients in $\mathfrak{m}_{S}$, clearly

$$
\epsilon+\left(\phi_{\bullet}^{\prime}, \psi_{\bullet}^{\prime}\right)=\epsilon\left(\phi \bullet+\phi_{\bullet}^{\prime}, \psi_{\bullet}+\psi_{\bullet}^{\prime}\right) .
$$

This proves our assertion.

Remark 1.7. After [11] was written and initially posted on the arXiv, I was informed by Michel Duflo that the 'Hard Lemma' (Proposition 0.2) of [11], which is at the basis of the construction of the Jacobi-Bernoulli complex, had been proved earlier, and used in a different Lie-theoretic context, in the Jussieu thesis of his student Emanuela Petracci (see Bull. Sci. Math. 127 (2003)).

\section{NORMAL ALGEBRA}

Let $X \rightarrow P$ be a closed embedding of schemes. In this section we will construct, in terms of resolutions, a differntial graded Lie algebra $\mathcal{N}_{X / P}$, called the normal algebra, which controls motions and deformations of $X$ in $P$. This will be an essential ingredient in the construction of the abstract tangent Lie algebra of a scheme (such as $X$ ). The algebra $\mathcal{N}_{X / P}$ is a realization of the general normal atom of a subscheme (cf. [11]), one which works best if $P$ itself is an affine or projective space. See [11] for more information on Lie atoms.

2.1. Resolutions. Thus let $X$ be a closed subscheme of a quasi-projective scheme $P$ and let $\mathcal{I}=\mathcal{I}_{X / P}$ denote the ideal sheaf of $X$ in $P$. Let

$$
\ldots \rightarrow F^{-1} \rightarrow F^{0} \rightarrow \mathcal{I}
$$

be a locally free resolution of $\mathcal{I}$. As is well-known, $F$ is locally unique up to quasiisomorphism. In fact, when $P$ is an open subset of a projective space (e.g. an affine or projective space), we may assume

$$
F^{i}=N_{i} \mathcal{O}_{P}\left(-m_{i}\right):=\bigoplus_{1}^{N_{i}} \mathcal{O}_{P}\left(-m_{i}\right)
$$

for some large integer $m_{i}$. By Hilbert's syzygy theorem the resolution $F$. will be finite, i.e. bounded. For $i=0$, each summand $\mathcal{O}\left(-m_{0}\right)$ corresponds to a generator $f_{\alpha}$ of $\mathcal{I}, \alpha=1, \ldots, N_{0}$. Similarly for $i<0$ each summand $\mathcal{O}\left(-m_{i}\right)$ corresponds to a syzygy $G_{\alpha}^{i}$, that is, a vector

$$
\left(g_{\alpha \beta}^{i}\right) \in N_{i+1} \mathcal{O}\left(m_{i}-m_{i+1}\right)
$$

such that

$$
d_{F^{i+1}}\left(\sum g_{\alpha \beta}^{i} G_{\beta}^{i+1}\right)=0 .
$$

By definition,

$$
d\left(G_{\alpha}^{i}\right)=\sum_{\beta} g_{\alpha \beta}^{i} G_{\beta}^{i+1} .
$$


For notational consistency, we also set $G_{\alpha}^{0}=e_{\alpha}$. Thus, for each $i \leq 0$ the $G_{\alpha}^{i}$ form a basis of $F^{i}$. Next, set $F^{1}=\mathcal{O}_{P}$ and let $F_{+}$be the complex in degrees $\leq 1$ given by

$$
\ldots \rightarrow F^{0} \stackrel{\epsilon}{\rightarrow} F^{1}
$$

(where $\epsilon: F^{0} \rightarrow F^{1}$ is the composite $F^{0} \rightarrow \mathcal{I} \rightarrow \mathcal{O}_{P}$ ); thus $F_{+}$is quasi-isomorphic to $\mathcal{O}_{X}[-1]$.

Suppose now that $P$ is open in a projective space. Note that the (symmetric) multiplication map on $\mathcal{O}_{X}$ gives rise to a map of complexes

$$
\mu: \bigwedge^{2} F_{+} \rightarrow F_{+}[-1]
$$

which turns $F \cdot[1]$ into a differential graded commutative $\mathcal{O}_{P}$ algebra. $\mu$ is defined by descending induction, starting with the obvious multiplication maps

$$
F^{1} \times F^{1} \rightarrow F^{1}, F^{0} \times F^{1} \rightarrow F^{0} .
$$

Set $G \cdot \bigwedge^{2} F_{+}$Assuming $\mu$ is defined as map of complexes down through degree $i, i \leq 1$, note that

$$
d_{F_{+}[-1]}^{i} \circ \mu^{i} \circ d_{G}^{i-1}=\mu^{i+1} \circ d_{G}^{i} \circ d_{G}^{i-1}=0
$$

As $G^{i-1}$ is a free module and $F_{\dot{+}}$ is exact, this implies that $\mu^{i} \circ d_{G}^{i-1}$ factors through a map

$$
\mu^{i-1}: G^{i-1} \rightarrow F^{i-2}
$$

so we have $\mu$ as map of complexes down through degree $i-1$.

For example, for the map $\mu$ constructed in this way, $\mu\left(e_{\alpha} \wedge e_{\beta}\right)$ will be the Koszul relation $f_{\alpha} e_{\beta}-f_{\beta} e_{\alpha}$.

Note next that,

$$
\operatorname{deg} \mu(A B)=\operatorname{deg}(A)+\operatorname{deg}(B)-1<\min (\operatorname{deg}(A), \operatorname{deg}(B))
$$

provided $\operatorname{deg}(A), \operatorname{deg}(B) \leq 0$. Then considering the flexibility we have in defining the various syzygy modules $F^{i}$, as well as $\mu$, by descending recursion, we may assume that

(*) whenever $A, B$ are in the standard basis that $F$ comes with, then so is $\mu(A B)$;

(i.e. if $A B$ yields the trivial relation, we just introduce a basis element of the appropriate degree corresponding to the trivial relation and assign $\mu(A B)$ to it).

2.2. Definition of normal atom. We will denote by $\mathfrak{g} \cdot(\mathcal{I})$ the differential graded Lie algebra $\operatorname{Hom}\left(F^{\cdot}, F^{\cdot}\right)$, whose term in degree $i$ is

$$
\bigoplus_{j} \operatorname{Hom}\left(F^{j}, F^{i+j}\right) \text {, }
$$

whose differential is given by: for

$$
\left(\phi^{\cdot}\right) \in \mathfrak{g l}^{i}(\mathcal{I}), \phi^{j} \in \operatorname{Hom}\left(F^{j}, F^{i+j}\right),
$$


set $d\left(\phi^{\cdot}\right)=(\psi \cdot)$, where

(2. 2.4)

$$
\psi^{j}=d(\phi)^{j}=\phi^{j-1} \circ d_{F^{j-1}}+(-1)^{i+1} d_{F^{i+j}} \circ \phi^{j}
$$

and whose bracket is the usual graded commutator, i.e. for $\left(\phi^{*}\right),\left(\psi^{*}\right)$ homogeneous,

$$
\left[\left(\phi^{*}\right),\left(\psi^{\cdot}\right)\right]=\left(\phi^{\cdot}\right) \circ\left(\psi^{*}\right)-(-1)^{\operatorname{deg}\left(\phi^{\cdot}\right) \operatorname{deg}\left(\psi^{\cdot}\right)}\left(\psi^{\cdot}\right) \circ\left(\phi^{\cdot}\right) \text {. }
$$

Note that $d \phi$ itself is the graded commutator $[(\phi), d]$. There is an obvious action map

$$
\mathfrak{g l}^{\mathfrak{l}}(\mathcal{I}) \times F^{\cdot} \rightarrow F^{\cdot}
$$

as well as a multiplication map induced by $\mu$ :

$$
F \cdot \times \mathfrak{g l} \cdot(\mathcal{I}) \rightarrow \mathfrak{g l} \cdot(\mathcal{I})[-1]
$$

As we saw in [11], there is a Lie atom called the normal atom to $X$ in $P$, denoted

$$
\mathcal{N}=\mathcal{N}_{X / P}=\mathfrak{g l}\left(\mathcal{I}<\mathcal{O}_{P}\right)=\mathfrak{g l}\left(\mathcal{O}_{P}>\mathcal{O}_{X}\right),
$$

which can be defined as the mapping cone of the evident map

$$
\mathfrak{g l} \cdot\left(F_{+}, F_{+}^{1}\right) \rightarrow \mathfrak{g l}\left(F_{+}^{1}\right)
$$

where $\mathfrak{g l} \cdot\left(F_{+}, F_{+}^{1}\right)$ is the subalgebra of $\mathfrak{g l} \cdot\left(F_{\dot{+}}\right)$ consisting of endomorphisms sending the subcomplex $F_{+}^{1}=\mathcal{O}_{P}$ to itself. Thus $\mathcal{N}$ is also equivalent to the mapping cone

$$
\mathfrak{g l}^{r}(\mathcal{I}) \rightarrow \operatorname{Hom}\left(F^{\cdot}, \mathcal{O}_{P}\right),
$$

in other words, to the sub-dgla of $\mathfrak{g l} \cdot\left(F_{\dot{+}}\right)$ consisting of endomorphisms vanishing on $F_{+}^{1}$. Simply put, we have

$$
\mathcal{N}=\operatorname{Hom} \cdot\left(F \cdot F_{+}\right)
$$

and it is just the sub-dgla of $\mathfrak{g l}\left(F_{\dot{+}}\right)$ consisting of endomorphisms that vanish on the term (and subcomplex) $F_{+}^{1}$. Thus $\mathcal{N}$ has a dgla structure compatible with its Lie atom structure. Note that $\mathcal{N}$ acts naturally on $F_{+}$. Moreover this action annihilates, hence preserves, the subcomplex $F_{+}^{1} \subset F_{+}$, hence induces an action on the quotient $F^{\cdot}=F_{+} / F_{+}^{1}$. Thus we have (mutually compatible) action pairings

$$
\mathcal{N} \times F_{+} \rightarrow F_{+}, \quad \mathcal{N} \otimes F^{\cdot} \rightarrow F^{\cdot}
$$

Also, the multiplication map $\mu$ induces a multiplication

(2. 2.10)

$$
F_{+} \times \mathcal{N} \rightarrow \mathcal{N}
$$

making $\mathcal{N}$ an $\mathcal{O}_{X}$-module.

In case $P$ is an affine space, because as complex (or derived category object) $\mathcal{N}$ can be identified with $\operatorname{Hom}\left(F^{*}, F_{+}\right)$(i.e. $R \operatorname{Hom}\left(\mathcal{I}, \mathcal{O}_{X}\right)$ ), it follows that the cohomology

$$
H^{i}(\mathcal{N})=\operatorname{Ext}^{i}\left(\mathcal{I}, \mathcal{O}_{X}\right) .
$$


Example 2.1. As a trivial example, consider the case where $X$ is a (Cartier) divisor in $P$, with (invertible) ideal $\mathcal{O}(-X)$. Then $\mathfrak{g l}(\mathcal{I})=\mathcal{O}_{P}$ and $\mathcal{N}$ reduces to the complex $\mathcal{O}_{P} \rightarrow \mathcal{O}_{P}(X)$, i.e. to $\mathcal{O}_{X}(X)[-1]$.

\subsection{Relative situation. Suppose}

$$
X \rightarrow Y \rightarrow Z
$$

are closed embeddings. Then we can choose a resolution $F$. for $\mathcal{I}_{X / Z}$ so that

$$
F^{i}=F_{1}^{i} \oplus F_{2}^{i},
$$

where $F_{1}$ is a resolution of $\mathcal{I}_{Y / Z}$, and $F_{2} \otimes \mathcal{O}_{Y} \sim_{\text {qis }} F_{2} \otimes F_{1+}$ is a resolution of $\mathcal{I}_{X / Y}$. Then we have an exact triangle

$$
\operatorname{Hom}\left(F_{2}, F_{+}\right) \rightarrow \operatorname{Hom}\left(F^{\cdot}, F_{+}\right) \rightarrow \operatorname{Hom}\left(F_{1}, F_{+}\right) \rightarrow
$$

and note that via

$$
F_{2+} \otimes \mathcal{O}_{Y} \sim_{\text {qis }} F_{i} \otimes F_{i+} \sim_{\text {qis }} F_{+},
$$

the first term in 2. 3.13 is quasi-isomorphic to

$$
\operatorname{Hom}_{Y}\left(F_{\dot{2}} \otimes \mathcal{O}_{Y}, F_{2+} \otimes \mathcal{O}_{Y}\right)=\mathcal{N}_{X / Y},
$$

while the third term is similarly quasi-isomorphic to

$$
\operatorname{Hom} \cdot\left(F_{1}, F_{1+}\right) \otimes \mathcal{O}_{X} .
$$

In that sense then, we have a 'normal atom sequence'

$$
\mathcal{N}_{X / Y} \rightarrow \mathcal{N}_{X / Z} \rightarrow \mathcal{N}_{Y / Z} \otimes \mathcal{O}_{X} \rightarrow
$$

2.4. Jacobi complex. As discussed in [11], there is a Jacobi complex associated to $\mathcal{N}$, denoted $J(\mathcal{N})$, which, as sheaf, lives on $P\langle\infty\rangle$, the space of finite subsets of $P$ in and takes the form

$$
J(\mathcal{N}): \quad \ldots \rightarrow \lambda^{2} \mathcal{N} \rightarrow \mathcal{N}
$$

where the term in degree $-m, \lambda^{m} \mathcal{N}$, is the $m$-th external alternating power of $\mathcal{N}$ and the differential is induced by the bracket pairing on $\mathcal{N}$. In fact, since $\mathcal{N}$ is exact off $X, J(\mathcal{N})$ is quasi-isomorphic to, and may be replaced by, its (topological) restriction on $X\langle\infty\rangle$. The $m$ th truncation

$$
J_{m}(\mathcal{N}): \quad \lambda^{m} \mathcal{N} \rightarrow \ldots \rightarrow \lambda^{2} \mathcal{N} \rightarrow \mathcal{N}
$$

lives on $X\langle m\rangle$. The Jacobi complex $J(\mathcal{N})$ admits a comultiplicative structure

$$
J(\mathcal{N}) \rightarrow \operatorname{Sym}^{2}(J(\mathcal{N})),
$$

which induces a $\mathbb{C}$-algebra structure on $\mathbb{C} \oplus \mathcal{H}^{0}(J(\mathcal{N}))^{*}$ and on

$$
R_{m}(\mathcal{N})=\mathbb{C} \oplus \mathcal{H}^{0}\left(J_{m}(\mathcal{N})\right)^{*} .
$$

Then

$$
\widehat{R}(\mathcal{N})=\lim _{14} R_{m}(\mathcal{N})
$$


coincides with the formal completion at $[X]$ of the local ring of Hilbert scheme of subschemes of $P$.

As an alternative to working on spaces such as $P\langle\infty\rangle$, one may replace $\mathcal{N}$ by a representative complex of groups or $\mathbb{C}$-vector spaces whose terms are acyclic, and in this case $J(\mathcal{N})$ can be replaced by the standard Jacobi complex where $\lambda^{i}$ is just the ordinary exterior power over $\mathbb{C}$. Such a representative can be taken to be the sections of an injective or soft resolution. However in most applications considered here, $X$ is an affine scheme and it suffices to replace $\mathcal{N}$ simply by the corresponding complex of global sections. We will refer to this as the non-sheafy version of $J(\mathcal{N})$ in the affine case.

2.5. Regular embeddings. One point which has to be noted is that if $X \rightarrow P$ is a regular embedding (of codimension $>1$ ), our normal atom differs from the usual normal sheaf

$$
N=\operatorname{Hom}\left(\mathcal{I}, \mathcal{O}_{X}\right) .
$$

In fact, since $\mathcal{N}$ is $\operatorname{RHom}\left(\mathcal{I}, \mathcal{O}_{X}\right)$, it is given $\operatorname{Hom}\left(F \cdot, \mathcal{O}_{X}\right)$, where $F$ is the Koszul complex. Since the differentials of $F$. have coefficients in $\mathcal{I}$, it follows that $\mathcal{N}$ is the complex with trivial differentials and terms

$$
\begin{array}{r}
\mathcal{N}^{i}=\bigwedge_{\mathcal{O}_{X}}^{i} N, i=1, \ldots, \operatorname{codim}(X, P) \\
=0 \text { otherwise. }
\end{array}
$$

Why is it sufficient to work just with $N$ ? We may reason as follows. The bracket of $\mathcal{N}$, restricted on $N$ (which itself is viewed as a complex, indeed a subcomplex of $\mathcal{N}$, via an injective resolution) has 2 components: one is a map

$$
\operatorname{Sym}^{2} N \rightarrow N[-1]
$$

while the other is a (locally determined) map

$$
\operatorname{Sym}^{2}(N) \rightarrow \mathcal{N}^{2}=\bigwedge^{2} N
$$

(note that $\bigwedge^{2}$ becomes $\operatorname{Sym}^{2}$ on $\mathcal{N}^{1}$ ). We claim the second map vanishes. Indeed, locally, a section of of $\bigwedge^{i} N$ lifts to a section of $F^{-(i-1)}$, and this yields an endomorphism of degree $i$ of $F_{+}$via interior multiplication. Moreover, (signed) commutator of endomorphisms, which yields the bracket on $\mathcal{N}$, corresponds to wedge product. Thus for local sections $a, b$ of $N$, the commutator is $a b+b a$ which corresponds to $a \wedge b+b \wedge a=0$. This corresponds to the well-known fact that 'local obstructions to deforming complete intersections vanish'. Therefore $N$ with induced bracket (2.5.15) is a sub-Lie atom of $\mathcal{N}$. This remark will be generalized below via the notion of reduced normal atom. 
2.6. Weak equivalence. The following definition will be useful

Definition 2.2. A homomorphism

(2. 6.16)

$$
\theta: \mathfrak{g}_{1} \rightarrow \mathfrak{g}_{2}
$$

of Lie atoms or dg Lie algebras is said to be a direct weak equivalence or diweq if it induces an isomorphism on $H^{i}, \forall i \leq 1$, and an injection on $H^{2}$. A general weak equivalence or weq is a composition of direct weak equivalences and their inverses. The relation of weak equivalence is the equivalence relation generated by the existence of a direct weak equivalence.

The discussion of the preceding subsection proves

Lemma 2.3. If $X \rightarrow P$ is a regular embedding then the normal atom $\mathcal{N}_{X / P}$ is weakly equivalent to the shifted normal bundle $N_{X / P}[-1]$ with trivial bracket.

The following observation is easy to prove but will be quite useful in what follows.

Lemma 2.4. A weak equivalence (2. 6.16) induces an isomorphism on deformation rings

$$
R_{m}\left(\mathfrak{g}_{1}\right) \simeq R_{m}\left(\mathfrak{g}_{2}\right) .
$$

For the normal atom $\mathcal{N}$ of a regular embedding, clearly $N[-1] \rightarrow \mathcal{N}$ is a weak equivalence.

Remark 2.5. If $X$ is smooth and $P$ is an open subscheme of a projective space, it is possible to identify the Lie atom $N$ as constructed above with the 'differential' normal atom $N^{\text {diff }}$, defined by the complex

$$
T_{P / X} \rightarrow T_{P} \sim N^{\text {diff }}[-1],
$$

where $T_{P / X}$ is the algebra of vector fields on $P$ tangent to $X$ along $X$ (see [11]). Briefly, the methods of the next section show that we can define a diagram

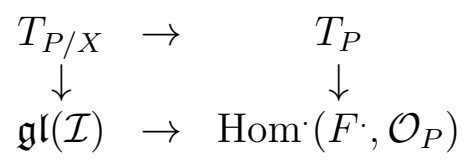

by making vector fields act on the equations of $X$. This diagram induces a quasiisomorphism of Lie atoms

$$
N^{\text {diff }}[-1] \rightarrow N .
$$

In this sense then, $\mathcal{N}$ is weakly equivalent to $N^{\text {diff }}[-1]$. 
2.7. Reduced normal atom. Consider now the case where $P$ is an open subscheme of an projective space, but $X \rightarrow P$ is arbitrary. Our purpose here is to show that the normal atom $\mathcal{N}$ can be replaced by another, weakly equivalent dgla $\mathcal{N}_{\text {red }}$ which is 'smaller' and more convenient for many applications. Essentially, $\mathcal{N}_{\text {red }}$ is obtained from $\mathcal{N}$ by removing the trivial Koszul syzygies, which play no role in deformation and obstruction theory. This construction is motivated by, and analogous to, the cotangent complex of a scheme, which goes back to Grothendieck.

Consider a resolution $F \cdot \stackrel{\epsilon}{\rightarrow} I$ as above, where $F^{0}$ is a free module on a basis $\left(e_{\alpha}\right)$ that maps to a system of generators $\left(f_{\alpha}\right)=\left(\epsilon\left(e_{\alpha}\right)\right)$ of $I$. Let $K_{0} \subset F^{0}$ denote the 'Koszul' submodule, generated by all elements of the form

$$
e_{\alpha \beta}=f_{\alpha} e_{\beta}-f_{\beta} e_{\alpha} .
$$

Note that if the $e_{\alpha}$ form a regular sequence, then $K_{0}=\operatorname{ker}(\epsilon)$. Let

$$
\ldots \rightarrow F_{0}^{-2} \rightarrow F_{0}^{-1} \rightarrow K_{0}
$$

be a free resolution of $K_{0}$, where $F_{0}^{-1}$ is a free module on generators $s_{\alpha \beta}$ that map to $e_{\alpha \beta}$. We may assume our resolution $F$. of $I$ is constructed in such a way that for each $i<0$, there is a splitting of $F^{i}$ into free modules $F^{i}=F_{0}^{i} \oplus F_{1}^{i}$. Such resolutions $F$. will be called standard. Thus any resolution is equivalent to a standard one. Note that, for a standard resolution $F$, the complex $F^{<0}$, itself a quotient of $F$ as well as $F_{+}$, fits in an exact sequence

$$
0 \rightarrow F_{0}^{<0} \rightarrow F^{<0} \rightarrow F_{1}^{<0} \rightarrow 0 .
$$

If the $e_{\alpha}$ form a regular sequence, then clearly $F_{1}^{<0}=0$.

Now the idea is that if we deform the subscheme $X \subset P$ by deforming its resolution, one might as well start with a standard resolution and deform the latter in the more restricted universe of standard resolutions, rater than all resolutions. This is accomplished by the dgla $\mathcal{N}_{\text {red }}$ that we now introduce.

Start with a standard resolution $F$ as above. Then define a map

(2. 7.20)

$$
\pi: \operatorname{Hom}\left(F^{0}, F_{+}^{i}\right) \rightarrow \operatorname{Hom}\left(F_{0}^{-1}, F_{+}^{i-1}\right) \subset \operatorname{Hom}\left(F^{-1}, F_{+}^{i-1}\right), \forall i \leq 1
$$

by the formula

$$
\pi\left(e_{\alpha}^{*} \otimes g\right)=(-1)^{i} \sum_{\beta} s_{\alpha \beta}^{*} \otimes \mu\left(e_{\beta} \otimes g\right), \forall g \in F^{i}
$$

where

$$
\mu: F^{0} \otimes F_{+}^{i} \rightarrow F_{+}^{i-1}
$$

is the graded multiplication (induced by the multiplication on $A_{X}$ as in (2.1.3)). One can easily check that

$$
\begin{gathered}
s_{\alpha \beta}=\mu\left(e_{\alpha} \otimes e_{\beta}\right) . \\
17
\end{gathered}
$$


In particular, $\mu\left(F^{0} \otimes F^{0}\right) \subset F_{0}^{-1}$. Now the commutative diagram (with left vertical maps id $\otimes d$ )

$$
\begin{array}{cccc}
F^{0} \otimes F_{+}^{-1} & & \\
& & & \\
& \downarrow & & \\
F^{0} \otimes F_{+}^{0} & \rightarrow & F^{-1} \\
& \downarrow & & \downarrow \\
F^{0} \otimes F_{+}^{0} & = & F^{0}
\end{array}
$$

shows that the composite

$$
F^{0} \otimes F^{-1} \stackrel{\text { id } \otimes d}{\rightarrow} F^{0} \otimes F^{0} \stackrel{\mu}{\rightarrow} F_{0}^{-1}
$$

factors through the kernel of $F_{0}^{-1} \rightarrow K_{0}$, hence we may assume that $\left.\mu\right|_{F^{0} \otimes F^{-1}}$ factors through $F_{0}^{-2}$. Then again the commutative diagram

$$
\begin{array}{cccc}
F^{0} \otimes F^{-2} & & \\
\downarrow & & \\
F^{0} \otimes F^{-1} & \rightarrow & F_{0}^{-2} \\
\downarrow & & \downarrow \\
F^{0} \otimes F^{0} & \rightarrow & F_{0}^{-1}
\end{array}
$$

shows may assume that $\left.\mu\right|_{F^{0} \otimes F^{-2}}$ factors through $F_{0}^{-3}$, etc. Thus, we may assume (2. 7.22)

$$
\mu\left(F^{0} \otimes F^{i}\right) \subset F_{0}^{i-1}, \ell \forall i \leq 0,
$$

and consequently

(2. 7.23$)$

$$
\pi\left(\operatorname{Hom}\left(F^{0}, F_{+}^{i}\right)\right) \subset \operatorname{Hom}\left(F_{0}^{-1}, F_{0}^{i-1}\right)
$$

We consider $\pi$ as an endomorphism of $\mathcal{N}$. Note that, trivially, $\pi^{2}=0$.

Lemma 2.6. $\pi$ is a Lie homomorphism.

Proof. It suffices to check this on $\operatorname{Hom}\left(F^{0}, F_{+}^{i}\right)$ and the only nontrivial case is $i=0$, in which case we must prove

$$
\pi\left(\left[e_{\alpha_{1}}^{*} e_{\gamma_{1}}, e_{\alpha_{2}}^{*} e_{\gamma_{2}}\right]\right)=\left[\pi\left(e_{\alpha_{1}}^{*} e_{\gamma_{1}}\right), \pi\left(e_{\alpha_{2}}^{*} e_{\gamma_{2}}\right)\right] .
$$

Suppose first that $\gamma_{1} \neq \alpha_{2}, \gamma_{2} \neq \alpha_{1}$, so the LHS is clearly zero. Then we compute the RHS:

$$
\sum_{\beta_{1}, \beta_{2}}\left[s_{\alpha_{1} \beta_{1}}^{*} s_{\beta_{1} \gamma_{1}}, s_{\alpha_{2} \beta_{2}}^{*} s_{\beta_{2} \gamma_{2}}\right]=s_{\alpha_{1} \alpha_{2}}^{*} s_{\gamma_{1} \gamma_{2}}-s_{\alpha_{2} \alpha_{1}}^{*} s_{\gamma_{2} \gamma_{1}}=0
$$

If $\gamma_{1}=\alpha_{2}, \gamma_{2} \neq \alpha_{1}$ then the RHS is

$$
\sum_{\beta} s_{\alpha_{1} \beta}^{*} s_{\beta \gamma_{2}}
$$

which clearly equals the LHS. Other cases are similar. 
Lemma 2.7. The map

$$
i_{F_{0}^{-1}}^{*} \circ d: \operatorname{Hom}\left(F^{0}, F_{+}^{i}\right) \rightarrow \operatorname{Hom}\left(F_{0}^{-1}, F^{i}\right)
$$

coincides with

$$
i_{F_{0}^{-1}}^{*} \circ(-d \circ \pi+\pi \circ d) .
$$

Proof. Pick $\phi=e_{\alpha}^{*} \otimes g \in \operatorname{Hom}\left(F^{0}, F^{i}\right)$. Then we have

$$
d \phi=\phi \circ d-(-1)^{i} d \circ \phi=-s_{\alpha \beta}^{*} f_{\beta} \otimes g-(-1)^{i} e_{\alpha}^{*} \otimes d g .
$$

Hence, the component of $\pi d \phi$ in $\operatorname{Hom}\left(F^{-1}, F^{i}\right)$ is

$$
(\pi d \phi)^{1, i+1}=s_{\alpha \beta}^{*} \otimes \mu\left(e_{\beta} \otimes d g\right) .
$$

On the other hand, since

$$
\pi(\phi)=(-1)^{i} s_{\alpha \beta}^{*} \mu\left(e_{\beta} \otimes g\right),
$$

we have

$$
d \pi(\phi)^{1, i}=s_{\alpha \beta}^{*} \otimes\left(f_{\beta} g+\mu\left(e_{\beta} \otimes d g\right)\right)
$$

Therefore,

$$
d \phi\left(s_{\alpha \beta}\right)=-d \pi \phi\left(s_{\alpha \beta}\right)+\pi d \phi\left(s_{\alpha \beta}\right), \forall \alpha, \beta
$$

which proves our assertion.

We exploit the lemma as follows. Set

$$
d^{\prime}=d+d \pi-\pi d: \mathcal{N} \rightarrow \mathcal{N} .
$$

Then it is immediate that

$$
\left(d^{\prime}\right)^{2}=d \pi d-d \pi d=0 .
$$

Therefore we may define a complex

$$
\mathcal{N}^{\prime}=\left(\mathcal{N}, d^{\prime}\right) .
$$

By the very definition of $d^{\prime}$, the map

$$
\mathrm{id}+\pi: \mathcal{N}^{\prime} \rightarrow \mathcal{N}
$$

is a map of complexes. The map is clearly an isomorphism, with inverse id $-\pi$. Now define subgroups of $\mathcal{N}^{\prime}$ as follows:

$$
{ }^{\operatorname{red}} \mathcal{N}=\bigoplus_{i>0, j} \operatorname{Hom}\left(F_{1}^{-i}, F_{+}^{j}\right) \oplus \bigoplus \operatorname{Hom}\left(F^{0}, F_{+}^{j}\right),
$$

$$
\mathcal{N}_{0}=\bigoplus_{i>0, j} \operatorname{Hom}\left(F_{0}^{-i}, F_{+}^{j}\right) .
$$

Note that when the $e_{\alpha}$ form a regular sequence,

$$
{ }^{\mathrm{red}} \mathcal{N}=\operatorname{Hom} \cdot\left(F^{0}, F_{+}\right) \sim \operatorname{Hom}\left(F^{0}, A_{X}\right)[-1] .
$$


Now Lemma 2.7 means precisely that the obvious splitting of $\mathcal{N}$ as group yields a splitting of the complex $\mathcal{N}^{\prime}$ :

$$
\mathcal{N}^{\prime}={ }^{\operatorname{red}} \mathcal{N} \oplus \mathcal{N}_{0} .
$$

Now, it is easy to check that ${ }^{\text {red }} \mathcal{N}$ is in fact a sub-dgla of $\mathcal{N}^{\prime}$. I claim next that id $+\pi$ is a Lie homomorphism on ${ }^{\text {red }} \mathcal{N}$, i.e. that

$$
\left[\phi_{1}+\pi\left(\phi_{1}\right), \phi_{2}+\pi\left(\phi_{2}\right)\right]=\left[\phi_{1}, \phi_{2}\right]+\pi\left(\left[\phi_{1}, \phi_{2}\right]\right) .
$$

Indeed it suffices to check this when $\phi_{1}, \phi_{2}$ are bihomogeneous, in which case, by (2. 7.23), it holds for trivial reasons except when $\phi_{1}, \phi_{2} \in \operatorname{Hom}\left(F^{0}, F^{0}\right)$. In this case, (2. 7.27) follows from the fact that $\pi$ is a Lie homomorphism (Lemma 2.6). The same lemma also shows that $d^{\prime}$ is a derivation, so that $\mathcal{N}^{\prime}$ is a dgla.

Now, since

$$
\mathcal{N}_{0} \sim \operatorname{Hom} \cdot\left(F_{0}^{<0}, A_{X}[-1]\right),
$$

this complex is acyclic in degrees $<2$. Therefore the inclusion $\mathcal{N}_{\text {red }} \rightarrow \mathcal{N}^{\prime}$, hence

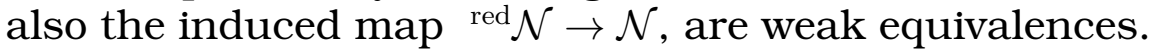

Also, it is easy to check that the usual argument showing that the resolution $F^{\cdot}$ is uniquely determined up to homotopy also shows that ${ }^{\text {red }} \mathcal{N}$ is uniquely determined up to dgla homotopy.

We summarize the foregoing discussion in the following statement.

Theorem 2.8. There exists a dgla ${ }^{\mathrm{red}} \mathcal{N}={ }^{\mathrm{red}} \mathcal{N}_{X / P}$, canonical up to dgla homotopy, together with a weq

$$
{ }^{\operatorname{red}} \mathcal{N} \rightarrow \mathcal{N}_{X / P}
$$

such that, whenever $X \rightarrow P$ is a regular embedding, we have

$$
{ }^{\mathrm{red}} \mathcal{N} \sim N_{X / P}=\operatorname{Hom}\left(I_{X}, A_{X}\right)[-1] .
$$

Note that if a subset of the generators $f_{\alpha}$ of $I$ form a regular sequence, they contribute to $\mathcal{N}_{\text {red }}$ only via $\operatorname{Hom}\left(F^{0}, F_{\dot{+}}\right) \sim \operatorname{Hom}\left(F^{0}, A_{X}\right)$. Combining this with (2. 3.14) above, we conclude the following

Corollary 2.9. If $X \rightarrow Y \rightarrow Z$ are closed embeddings with $Y \rightarrow Z$ regular, then there is an exact sequence

$$
0 \rightarrow{ }^{\text {red }} \mathcal{N}_{X / Y} \rightarrow{ }^{\text {red }} \mathcal{N}_{X / Z} \rightarrow N_{Y / Z}[-1] \otimes A_{X} \rightarrow 0 .
$$

Next, note that if $f \in A_{P}$ and $\bar{f} \in A_{X}$ is its image, then we have a closed embedding of principal open affines

$$
X_{\bar{f}} \rightarrow P_{f}
$$

and a resolution of $X \rightarrow P$ yields one of $X_{\bar{f}} \rightarrow P_{f}$, therefore we have a dgla quasi-isomorphism

$$
\left({ }^{\operatorname{red}} \mathcal{N}_{X / P}\right)_{\bar{f}} \sim{ }^{\operatorname{red}} \mathcal{N}_{X_{\bar{f}} \rightarrow P_{f}} .
$$

In particular, if $X_{\bar{f}} \rightarrow P_{f}$ is regular, then ${ }^{\text {red }} \mathcal{N}_{X / P}$ is acyclic in degree $>1$ over $X_{\bar{f}}$. So we conclude 
Corollary 2.10. For $i>1, H^{i}\left({ }^{\operatorname{red}} \mathcal{N}\right)$ is supported on the set of points where $X \rightarrow P$ is not a regular embedding.

Remark 2.11. To fix ideas, we have worked in this section in the setting of coherent sheaves. However when $P$ is an affine or projective space, there is no difficulty in shifting from the sheafy setting to the module (resp. graded module) setting, using the standard exact functors $\Gamma$ (in the affine case) or $\Gamma_{*}$ (in the projective case).

\section{Affine schemes: Tangent Atom}

Our purpose eventually is to define, for any algebraic scheme $X$, a Lie atom $\mathcal{T}_{X}$ called the tangent atom of $X$, that will play a role in deformation theory akin to that of tangent algebra for a smooth scheme. We begin in this section with the case where $X$ is specified as embedded as closed subscheme in a fixed affine space $P=\mathbb{A}^{n}$. The complex $\mathcal{T}_{X}=\mathcal{T}_{X}(P)$ that we construct will depend on the embedding $X \rightarrow P$ - as well as a number of other choices- but, as we shall see, the weak equivalence class of $\mathcal{T}_{X}(P)$ will be independent of all the choices, and will depend- functorially- only on the isomorphism class of $X$. Moreover, $\mathcal{T}_{X}(P)$ will in fact be (weakly equivalent to) a dgla, rather than a general Lie atom.

To fix ideas, we will be working in this section in the setting of (finitely generated) modules over the affine coordinate ring $A_{P}$. There is no difficulty in carrying the construction to the setting of coherent sheaves over $P$, but we will not need this. In any event, the extension to the global (non-affine) setting is far from straightforward and will be taken up in the next section.

3.1. Construction of differential graded group. In this subsection we will construct the differential graded group underlying the tangent dgla $\mathcal{T}_{X}$. The proof that this forms a complex, as well as the construction and properties of the Lie bracket, will be deferred to the next subsection.

Now our construction of the tangent atom $\mathcal{T}_{X}$ is motivated by the fact that for $X$ smooth, its tangent algebra is quasi-isomorphic to the complex (of Lie atoms)

$$
\mathcal{I}_{X} \otimes T_{P} \rightarrow T_{P} \rightarrow N_{X / P} .
$$

There is another Lie algebra denoted $T_{X / P}$ of ambient vector fields on $P$ tangent to $X$ along $X$ (or what is the same, derivations on $A_{P}$ taking the ideal $I_{X}$ to itself), which itself is the mapping cone of $T_{P} \rightarrow N_{X / P}$, so that $T_{X}$ is the mapping cone of $I_{X} \otimes T_{P} \rightarrow T_{X / P}$. The plan is to construct a complex like (3. 1.1) in the general case, with $\mathcal{I}_{X}$ replaced, naturally enough, by a free resolution of itself, and $N_{X / P}$ replaced by (a convenient model of) the shifted normal atom $\mathcal{N}_{X / P}[1]$, or its reduced version ${ }^{\mathrm{red}} \mathcal{N}_{X / P}[1]$. Indeed, everything but the second arrow has already been constructed. Thus it remains to construct (something like) the second arrow, though in the singular case one cannot expect this arrow to be surjective. 
Thus, the tangent atom

$$
\mathcal{T}=\mathcal{T}_{X}=\mathcal{T}_{X}(P)
$$

will be a complex $\mathcal{T}$, depending on the embedding $X \rightarrow P$. Alongside $\mathcal{T}$, we will be constructing a complex $\mathcal{T}_{\dot{X} / P}$ analogous to $T_{X / P}$ in the smooth case. To define $\mathcal{T}$, fix a (finite) free resolution $F$ in degrees $\leq 0$ of the ideal $I=I_{X / P}$ (non-sheafy version), as above, with $F_{+}$the corresponding resolution in degrees $\leq 1$ of $A_{X}$, with $F_{+}^{1}=A_{P}$. Recall that the normal atom

$$
\mathcal{N}=\mathcal{N}_{X / P}=\operatorname{Hom} \cdot\left(F^{\cdot}, F_{+}\right) .
$$

Thus

$$
\mathcal{N}^{i}=\operatorname{Hom}^{i}\left(F^{\cdot}, F^{\cdot}\right) \oplus \operatorname{Hom}\left(F^{-i+1}, A_{P}\right)=\mathfrak{g l}^{i}(I) \oplus \check{F}^{-i+1},
$$

where, as before, we identify

$$
\mathfrak{g l}^{i}(\mathcal{I})=\mathfrak{g l}^{i}\left(F^{\cdot}\right)=\bigoplus_{j} \operatorname{Hom}\left(F^{j}, F^{i+j}\right) .
$$

We view $\mathcal{N}$ as the sub-dgla of $\mathfrak{g l} \cdot\left(F_{\dot{+}}\right)$ consisting of elements annihilating the subcomplex $F_{+}^{1}$ of $F_{+}$. Now the terms of $\mathcal{T}$. are defined as follows

$$
\mathcal{T}^{i}=T_{P} \otimes F_{+}^{i+1} \oplus \mathcal{N}^{i} .
$$

More explicitly,

$$
\begin{aligned}
\mathcal{T}^{i} & =\left(T_{P} \otimes F_{+}^{i+1}\right) \oplus \mathfrak{g l}^{i}(I), \quad i \leq 0, \\
& =\mathfrak{g l}^{i}(I) \oplus \operatorname{Hom}\left(F^{-i+1}, A_{P}\right), \quad i \geq 1 .
\end{aligned}
$$

(3. 1.4)

So, schematically,

$$
\mathcal{T}_{X}: \ldots \rightarrow \begin{gathered}
\mathfrak{g l}^{-1}(I) \\
\oplus \\
\left(T_{P} \otimes F^{0}\right)
\end{gathered} \rightarrow \begin{gathered}
\mathfrak{g l}^{0}(I) \\
T_{P}
\end{gathered} \rightarrow \underset{\tilde{F}^{0}}{\oplus} \rightarrow \ldots
$$

(with the middle term in degree 0 ). Thus as graded group, $\mathcal{T}_{X}$ in the affine case is just $\mathcal{N} \oplus\left(T_{P} \otimes F_{+}\right)$. The analogous complex $\mathcal{T}_{\dot{X} / P}$ is constructed similarly, with $T_{P} \otimes F_{+}$replaced by $T_{P}$, i.e.

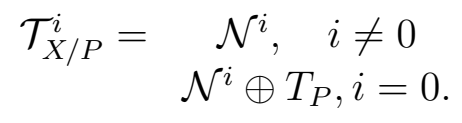

Note that all $\mathcal{T}^{i}$ are finitely generated locally free (even globally free, if $P$ is affine space) sheaves, and all but finitely many vanish. We will define the differentials on $\mathcal{T}$. in such a way that all components with source (resp. target) in $\mathcal{N}$ (resp. $T_{P} \otimes F_{\dot{+}}$ ) will coincide with the original ones from $\mathcal{N}$ (resp. $\left.T_{P} \otimes F_{\dot{+}}\right)$, so that there will be a exact sequences of complexes

$$
\begin{gathered}
0 \rightarrow \mathcal{N} \rightarrow \mathcal{T} \rightarrow T_{P} \otimes F_{+} \rightarrow 0, \\
0 \rightarrow \mathcal{N} \rightarrow \mathcal{T}_{P / X} \rightarrow T_{P} \rightarrow 0 . \\
22
\end{gathered}
$$


Thus what really needs to be defined is the 'mixing' part, i.e. the differential components going from $T_{P} \otimes F_{+}$to $\mathcal{N}$.

Now explicitly, the various components of the differentials $d^{i}: \mathcal{T}^{i} \rightarrow \mathcal{T}^{i+1}$ are defined as follows.

- For $i=0$, the components of $d^{0}$ are:

- the map $\mathfrak{g l}^{0}(I) \rightarrow \mathfrak{g l}^{1}(I)$ is the differential of $\mathfrak{g l} \cdot(I)$;

- the map $\mathfrak{g l}^{0}(I) \rightarrow \operatorname{Hom}\left(F^{0}, A_{P}\right)$ sends a graded map $(\phi)$ to $\epsilon \circ \phi^{0}$;

- let $\left(e_{\alpha}\right)$ be a basis for the free module $F^{0}$ and $f_{\alpha}=\epsilon\left(e_{\alpha}\right)$ the corresponding generator of $I$, so that

$$
\epsilon=\sum_{\alpha} f_{\alpha} e_{\alpha}^{*}
$$

then the map

$$
T_{P} \rightarrow \operatorname{Hom}\left(F^{0}, A_{P}\right)
$$

sends a section (vector field) $v$ to

(3. 1.6)

$$
d^{0}(v)=\left(v\left(f_{a}\right)\right)=\sum_{\alpha} v\left(f_{\alpha}\right) e_{\alpha}^{*}
$$

- the map

$$
u=(u): T_{P} \rightarrow \mathfrak{g l}^{1}(I)=\bigoplus_{j} \operatorname{Hom}\left(F^{j} \rightarrow F^{j+1}\right)
$$

has $j$-th component $u^{j}$ defined as follows. Let $\left(G_{\alpha}^{j+1}\right)$ be a basis of $F^{j+1}$ and $\left(G_{\beta}^{j}\right)$ be a basis of $F^{j}$. Thus each $G_{\beta}^{j}$ is a syzygy between the $G_{\alpha}^{j+1}$ and may be written as a vector $G_{\beta}^{j}=\left(g_{\beta \alpha}^{j}\right)$ with each $g_{\beta \alpha}^{j} \in A_{P}$ and

$$
\begin{array}{r}
\sum_{\alpha} g_{\beta \alpha}^{j} d^{j+1}\left(G_{\alpha}^{j+1}\right)=\sum_{\alpha} g_{\beta \alpha}^{j} g_{\alpha \eta}^{j+1} G_{\eta}^{j+2}=0, \quad j+1<0 \\
\sum_{\alpha} g_{\beta \alpha} \epsilon\left(e_{\alpha}\right)=\sum_{\alpha} g_{\beta \alpha} f_{\alpha}=0, \quad j+1=0
\end{array}
$$

In fact the second equation becomes a special case of the first, for $j+1=0$ if we take for $\left(G_{\eta}^{1}\right)$ the standard basis (1) of $A_{P}=F_{+}^{1}$. Note that (3. 1.7) is equivalent to the scalar identity

$$
\sum_{\alpha} g_{\beta \alpha}^{j} g_{\alpha \eta}^{j+1}=0, \forall \beta, \eta
$$

Then set

$$
u^{j}(v)=\sum_{\alpha, \beta} v\left(g_{\beta \alpha}^{j}\right) G_{\beta}^{j *} G_{\alpha}^{j+1} .
$$

Thus, all in all, the differential

$$
d: T_{P} \rightarrow \mathcal{N}^{1}
$$


is defined by

(3. 1.10)

$$
d(v)=\sum_{j \leq 0, \alpha, \beta} v\left(g_{\beta \alpha}^{j}\right) G_{\beta}^{j *} G_{\alpha}^{j+1} .
$$

This may be explained by noting that $v \in T_{P}$ defines in an obvious way a linear map $I_{X} \rightarrow A_{X}=A_{P} / I / X$. Then $d(v)$ is just the extension of this to a map of resolutions $F \cdot F_{+}$. Another explanation is this. Since $F_{+}$is endowed with a specified basis, the derivation $v$ of $A_{P}$ extends to an additive termwise endomorphism (in fact, an $A_{P^{-}}$ derivation compatible with the derivation $v$ of $A_{P}$ ) of $F_{+}$; then $d(v)$ is just the commutator of $v$ with the differential $d$, i.e.

(3. 1.11)

$$
d(v)=[v, d]
$$

(note that a priori $[v, d]$ vanishes on $F_{+}^{1}$ because $d$ does, $v$ kills 1 , and $[v, d]$ is linear, as the commutator of a derivation and a linear map).

- for $i<0$, the components of $d^{i}$ are:

- the map $\mathfrak{g l}^{i}(I) \rightarrow \mathfrak{g l}^{i+1}(I)$ is the $\mathfrak{g l}(I)$-differential;

- the map $F_{+}^{i+1} \otimes T_{P} \rightarrow F_{+}^{i+2} \otimes T_{P}$ is $d_{F_{+}} \otimes \mathrm{id}_{T_{P}}$;

- the map

$$
u=(u): F^{i+1} \otimes T_{P} \rightarrow \mathfrak{g l}^{i+1}(I)=\bigoplus_{j} \operatorname{Hom}\left(F^{j}, F^{j+i+1}\right)
$$

is given by

(3. 1.12)

$$
u^{j}(G \otimes v)=\sum_{\alpha, \beta} v\left(g_{\beta \alpha}^{j}\right) G_{\beta}^{j *} \mu\left(G \otimes G_{\alpha}^{j+1}\right)
$$

where

$$
\mu: F_{+}^{a} \otimes F_{+}^{b} \rightarrow F_{+}^{a+b-1}
$$

is the pairing obtained as the extension of the multiplication map on $A_{X}$ to its free $A_{P}$-resolution (cf. (2. 1.3)).

Note that, compared to the definition of $d v \in \mathcal{N}^{1}$ above, we can write this component in the form $\mu(G . d v)$ where the $\mu$ multiplication refers to the natural extension of $\mu$

$$
\begin{gathered}
\mu: F^{k} \times \mathcal{N}^{i} \rightarrow \mathcal{N}^{i+k-1} \\
G_{\gamma}^{k} \times G_{\beta}^{j *} \otimes G_{\alpha}^{j+i} \mapsto G_{\beta}^{j *} \otimes \mu\left(G_{\gamma}^{k} G_{\alpha}^{i+j}\right) .
\end{gathered}
$$

As in (3. 1.11), this definition may be explained by the remark that $G \otimes v$ defines a derivation of degree $i-1$ of $F_{+}$as $A_{P}$ module, and $d(G \otimes v)$ is just the commutator:

(3. 1.13)

$$
d(G \otimes v)=[G \otimes v, d]
$$

- For $i>0$ :

- The map $\mathfrak{g l}^{i}(I) \rightarrow \mathfrak{g l}^{i+1}(I)$ is the $\mathfrak{g l} \cdot(I)$-differential; 
- the map $\mathfrak{g l}^{i}(I) \rightarrow \operatorname{Hom}\left(F^{-i}, A_{P}\right)$ sends a graded map $(\phi)$ to $\epsilon \circ \phi^{i}$ where $\phi^{i}: F^{-i} \rightarrow F^{0}$ is the degree- $i$ component of $\phi$.

All other components are zero.

This completes the construction of the differential on $\mathcal{T}_{X}$; we will shortly prove that the square of this differential is zero, making $\mathcal{T}_{X}$ a complex.

The differentials of the complex $\mathcal{T}_{X / P}$ are defined compatibly with $\mathcal{T}_{X}$, so that $\mathcal{T}_{X / P}$ will be a subcomplex of $\mathcal{T}_{X}$. Thus (modulo verifying that $\mathcal{T}_{X}, \mathcal{T}_{X / P}$ are complexessee below), there is a short exact sequence of complexes (exact triangle)

(3. 1.14)

$$
0 \rightarrow T_{P} \otimes F^{\cdot} \rightarrow \mathcal{T}_{X / P} \rightarrow \mathcal{T}_{X} \rightarrow 0
$$

Example 3.1. Continuing with example 2.1, suppose $X$ is a hypersurface with equation $f$ of degree $d$ in $P=\mathbb{C}^{n}$. As we have seen, $\mathcal{N}_{X / P}$ can be identified cohomologically with

$$
A_{P}(d) / f A_{P} \simeq A_{X},
$$

or more precisely with the complex

$$
A_{P} \stackrel{f}{\rightarrow} A_{P}(d) .
$$

The tangent algebra $\mathcal{T}_{X}$ is the complex

$$
T_{P}(-d) \stackrel{\left(L_{f},-e_{f}\right)}{\rightarrow} T_{P} \oplus A_{P} \stackrel{\left(e_{f}, L_{f}\right)}{\rightarrow} A_{P}(d)
$$

where $L_{f}$ is left (post) multiplication by $f$ and $e_{f}$ is evaluation on $f$. Thus the map $T_{P} \rightarrow A_{P}(d)$ sends a vector field $\partial / \partial X_{i}$ to $\partial f / \partial X_{i}$.

If $f$ is nondegenerate, this map yields an injection

$$
T_{P} / f T_{P} \rightarrow \mathcal{N}_{X / P}=A_{P}(d) / f A_{P} .
$$

Thus, in this case, $\mathcal{T}_{X}$ can be identified cohomologically with a shift of the Milnor algebra

$$
A(f)=\mathbb{C}\left[X_{1}, \ldots, X_{n}\right] /\left(f, \partial f / \partial X_{1}, \ldots, \partial f / \partial X_{n}\right)
$$

(which is finite-dimensional iff $X$ has isolated singularities).

3.2. Construction and basic properties of dgla. In this subsection we will construct $\mathcal{T}_{X}$ and $\mathcal{T}_{X / P}$ as dgla's and establish their basic properties and interrelation.

In what follows we shall be working in the derived category of coherent sheaves on $P$, and in particular identify a sheaf of modules, e.g. $A_{X}$, with a free resolution of itself. Also, if no confusion is likely, we shall usually make no distinction between a finitely generated module and the associated coherent sheaf, e.g. $A_{X}$ and $A_{X}$.

The main results of this subsection are summarized in the following.

Theorem 3.2. $\quad$ (i) $\mathcal{T}_{\dot{X}}$ and $\mathcal{T}_{\dot{X} / P}$ as defined above are complexes and admit structures of dgla; as such, both act on $A_{X} ; \mathcal{T}_{X}$ is also an $A_{X^{-}}$module. 
(ii) $\mathcal{T}_{X / P}$ is a subcomplex of $\mathcal{T}_{X}$ and The natural map $\mathcal{T}_{X / P} \rightarrow \mathcal{T}_{X}$ is a dgla homomorphism whose mapping cone is quasi-isomorphic as Lie atom to $I[1] \otimes T_{P}=F \cdot[1] \otimes T_{P}$.

(iii) Endowing $F \cdot \otimes T_{P} \sim I \otimes T_{P}$ with its natural Lie algebra structure as subalgebra of $T_{P}$, there is a Lie homomorphism $J: F \cdot \otimes T_{P} \rightarrow \mathcal{T}_{X / P}$ which realizes $F \cdot \otimes T_{P}$ as Lie ideal in $\mathcal{T}_{X / P}$, such that the quotient (= mapping cone of $J$ ) is $\mathcal{T}_{X}[-1]$.

(iv) The weak equivalence class of $\mathcal{T}_{\dot{X}}$ is independent of the embedding $X \rightarrow P$ and is functorial in $X$, in the following sense. To a morphism of affine schemes

$$
f: X \rightarrow Y
$$

is associated a map of Lie atoms

$$
d f: \mathcal{T}_{X} \rightarrow f^{!} \mathcal{T}_{Y}
$$

such that the pair

$$
\begin{gathered}
X \mapsto \text { weak equivalence class of } \mathcal{T}_{X} \\
f \mapsto d f
\end{gathered}
$$

is a functor from the category of affine schemes over $\mathbb{C}$ to that of weak equivalence classes of dgla's and dgla homomorphisms over $\mathbb{C}$.

Proof. The proof is somewhat long, so we break it into steps.

3.2.1. $\mathcal{T}_{\dot{X} / P}$ is a complex.

This means $d^{i+1} \circ d^{i}=0$. For all $i \neq 0$ this vanishing is obvious from the analogous property for the normal atom $\mathcal{N}^{\cdot}=\mathcal{N}_{\dot{X} / P}$. For $i=0$, the vanishing of $d^{1} \circ d^{0}$ on the $\mathfrak{g l}^{0}=\mathcal{N}^{0}$ summand of $\mathcal{T}_{X / P}^{0}$ similarly follows from the corresponding fact for the normal atom $\mathcal{N}$.

For the other summand $T_{P}$ of $\mathcal{T}_{X / P}^{0}$, consider a section $v$. Then $d^{1} \circ d^{0}(v)$ has components in $\operatorname{Hom}\left(F^{i}, F_{+}^{i+2}\right)$ for all $i \leq-1$, and we claim all of them vanish. We focus first on the case $i=-1$, i.e. we will show that the component of $d^{1} \circ d^{0}(v)$ in $\operatorname{Hom}\left(F^{-1}, F_{+}^{1}\right)=\operatorname{Hom}\left(F^{-1}, A_{P}\right)$ vanishes, as the proof for the other components is similar. The latter component is a sum of 2 terms, corresponding to 2 components $d^{00}(v), d^{01}(v)$ of $d^{0}(v)$. The first component $d^{00}(v)$ is the map

$$
\begin{gathered}
F^{0} \rightarrow A_{P}, \\
e_{\alpha} \mapsto v\left(f_{\alpha}\right)
\end{gathered}
$$

taking the basis element $e_{\alpha}$ corresponding to the generator $f_{\alpha}$ to $v\left(f_{\alpha}\right)$. Then the component of $d^{1}\left(d^{00}(v)\right)$ in $\operatorname{Hom}\left(F^{-1}, A_{P}\right)$ is the map

$$
\begin{aligned}
F^{-1} & \rightarrow A_{P} \\
G_{\beta}^{-1}=\left(g_{\beta \alpha}^{-1}\right) & \mapsto \sum_{\alpha} g_{\beta \alpha}^{-1} v\left(f_{\beta}\right)
\end{aligned}
$$


where $G_{\beta}^{-1}=\left(g_{\beta \alpha}^{-1}\right)$ is a basis element of $F^{-1}$ which, being a syzygy, satisfies

$$
\sum_{\beta} g_{\beta \alpha}^{-1} f_{\beta}=0
$$

The other component $d^{01}(v)$ of $d^{0}(v)$ is the map

$$
\begin{gathered}
F^{-1} \rightarrow F^{0}, \\
G_{\beta}^{-1} \mapsto \sum_{\alpha} v\left(g_{\beta \alpha}^{-1}\right) e_{\beta}
\end{gathered}
$$

and then the component of $d^{1}\left(d^{01}(v)\right)$ in $\operatorname{Hom}\left(F^{-1}, A_{P}\right)$ is the map

$$
G_{\beta}^{-1} \mapsto \sum_{\alpha} v\left(g_{\beta \alpha}^{-1}\right) f_{\beta} .
$$

Thus in total, the component of $d^{1} d^{0}(v)$ in $\operatorname{Hom}\left(F^{-1}, F^{0}\right)$ is the map

$$
\begin{array}{r}
G_{\beta}^{-1} \mapsto \sum_{\alpha} g_{\beta \alpha}^{-1} v\left(f_{\beta}\right)+\sum_{\alpha} v\left(g_{\beta \alpha}^{-1}\right) f_{\beta} \\
=v\left(\sum_{\alpha} g_{\beta \alpha}^{-1} f_{\beta}\right)=0 .
\end{array}
$$

The vanishing of the component of $d^{1} \circ d^{0}(v)$ in $\operatorname{Hom}\left(F^{i}, F_{+}^{i+2}\right)$ for each $i<-1$ follows similarly from the derivation property of $v$ and the characteristic property of syzygies (3. 1.9): thus, the component in question is just

$$
\begin{array}{r}
\sum_{\beta, \alpha, \eta}\left(g_{\beta \alpha}^{i} v\left(g_{\alpha \eta}^{i+1}\right)+v\left(g_{\beta \alpha}^{i}\right) g_{\alpha \eta}^{i+1}\right) G_{\beta}^{i *} G_{\eta}^{i+2} \\
=\sum_{\beta, \alpha, \eta} v\left(g_{\beta \alpha}^{i} g_{\alpha \eta}^{i+1}\right) G_{\beta}^{i *} G_{\eta}^{i+2}=0
\end{array}
$$

This completes the verification that $\mathcal{T}_{\dot{X} / P}$ is a complex.

A more abstract, less explicit proof that $d^{2}(v)=0$, based on (3. 1.11), can be given as follows:

$$
d^{2}(v)=[[v, d], d]=[v,[d, d]]-[d,[v, d]]
$$

the latter by the Jacobi identity. Now $[d, d]=d^{2} / 2=0$ and $[d,[v, d]]=[[v, d], d]$ because $d$ and $[v, d]$ both have degree 1 . Therefore

$$
[[v, d], d]=-[[v, d], d]
$$

hence $d^{2}(v)=[[v, d], d]=0$. 


\subsection{2. $\mathcal{T}_{X}$ is a complex.}

Now given that that $\mathcal{T}_{\dot{X} / P}$ is a complex, proving the same for $\mathcal{T}_{\dot{X}}$ amounts to showing that $d^{k} \circ d^{k-1}$ vanishes on the summand $F^{k} \otimes T_{P}$ of $\mathcal{T}_{X}^{k-1}$, for all $k \leq 0$. To bring out the idea, we will first work out the case $k=0$ as other cases are only notationally more complicated. Thus for a section $s=e_{\beta} \otimes v$ of $F^{0} \otimes T_{P}, d^{-1}(s)$ has 2 components: one in $T_{P}$, viz.

$$
\begin{gathered}
f_{\beta} v \in T_{P}, \quad \text { and } \\
-\bigoplus_{i \leq 0, \gamma, \alpha} g_{\gamma}^{i *} v\left(g_{\alpha \gamma}^{i}\right) \mu\left(e_{\beta} g_{\alpha}^{i+1}\right) \in \bigoplus \operatorname{Hom}\left(F^{i}, F^{i}\right) .
\end{gathered}
$$

Now applying $d^{0}$ to this, we get components in $\operatorname{Hom}\left(F^{i}, F_{+}^{i+1}\right)$ for all $i \leq 0$, and we claim they are all zero There are 2 cases. First, $i=0$. This component gets just 2 contributions, one from $T_{P}$, viz

$$
\sum_{\gamma} e_{\gamma}^{*} f_{\beta} v\left(f_{\gamma}\right),
$$

and one from $\operatorname{Hom}\left(F^{0}, F^{0}\right)$ (via post-composing with $d_{F_{+}}$), which is just

$$
-\sum_{\gamma} e_{\gamma}^{*} f_{\beta} v\left(f_{\gamma}\right),
$$

so the total is zero.

Next, take $i<0$. Then we get 3 contributions in $\left.\operatorname{Hom}\left(F^{i}, F^{i+1}\right)\right)$ : one from $T_{P}$, which equals

$$
I: \sum_{\gamma, \alpha} f_{\beta} v\left(g_{\gamma \alpha}^{i}\right) G_{\gamma}^{i *} G_{\alpha}^{i+1},
$$

one from $\operatorname{Hom}\left(F^{i}, F^{i}\right)$ (via post-composing with $d_{F}$.), which equals

$$
\begin{gathered}
I I:-\sum_{\gamma, \alpha} G_{\gamma}^{i *} v\left(g_{\gamma \alpha}^{i}\right) d_{F} \cdot\left(\mu\left(e_{\beta} G_{\alpha}^{i+1}\right)\right) \\
=-\sum_{\gamma, \alpha} G_{\gamma}^{i *} v\left(g_{\gamma \alpha}^{i}\right)\left(f_{\beta} G_{\alpha}^{i+1}+\sum_{\delta} g_{\alpha \delta}^{i+1} \mu\left(e_{\beta} G_{\delta}^{i+2}\right)\right)=: I I_{1}+I I_{2}
\end{gathered}
$$

plus a third one from $\operatorname{Hom}\left(F^{i+1}, F^{i+1}\right)$ (via pre-composing with $d_{F}$.), which equals

$$
\begin{aligned}
I I I & :-\sum_{\gamma, \alpha} G_{\alpha}^{i+1 *} v\left(g_{\alpha \delta}^{i+1}\right) \mu\left(e_{\beta} G_{\delta}^{i+2}\right) \circ d_{F}^{i} . \\
& =-\sum_{\gamma, \alpha, \delta} G_{\gamma}^{i *} g_{\gamma \alpha}^{i} v\left(g_{\alpha \delta}^{i+1}\right) \mu\left(e_{\beta} G_{\delta}^{i+2}\right)
\end{aligned}
$$

Now clearly $I+I I_{1}=0$; and because of the derivation property of $v$ and the characteristic syzygy property (3. 1.9), we have $I I_{2}+I I I=0$ as well. Therefore the total is zero. 
Now in the general case of $k \leq 0$, we consider a section

$$
s=G_{\beta}^{k} \otimes v \in F^{k} \otimes T_{P},
$$

and we need similarly to show the components of $d^{2}(s)$ in $\operatorname{Hom}\left(F^{i}, F_{+}^{i+k+1}\right)$ vanish for all $i \leq 0$. Again there are 2 cases. For $i=0$ there are the 2 contributions

$$
\sum_{\gamma} g_{\beta \alpha}^{k} v\left(f_{\gamma}\right) e_{\gamma}^{*} G_{\alpha}^{k+1}
$$

and

$$
-\sum_{\gamma} g_{\beta \alpha}^{k} v\left(f_{\gamma}\right) e_{\gamma}^{*} G_{\alpha}^{k+1}
$$

which cancel out. Then for $i<0$ there are again 3 contributions. First via $F^{k+1} \otimes T_{P}:$

$$
I: \sum_{\gamma, \alpha, \eta} g_{\beta \eta}^{k} v\left(g_{\gamma \alpha}^{i}\right) G_{\gamma}^{i *} \mu\left(G_{\alpha}^{i+1} G_{\eta}^{k+1}\right),
$$

second via $\operatorname{Hom}\left(F^{i}, F^{i+k}\right)$ :

$$
\begin{gathered}
I I:-\sum_{\gamma, \alpha \eta} G_{\gamma}^{i *} v\left(g_{\gamma \alpha}^{i}\right) d_{F} \cdot\left(\mu\left(G_{\beta}^{k} g_{\alpha}^{i+1}\right)\right) \\
=-\sum_{\gamma, \alpha \eta} G_{\gamma}^{i *} v\left(g_{\gamma \alpha}^{i}\right)\left(g_{\beta \eta}^{k} \mu\left(G_{\eta}^{k+1} G_{\alpha}^{i+1}\right)+\sum_{\delta} g_{\alpha \delta}^{i+1} \mu\left(G_{\beta}^{k} G_{\delta}^{i+2}\right)\right)=: I I_{1}+I I_{2}
\end{gathered}
$$

and third via $\operatorname{Hom}\left(F^{i+1}, F_{+}^{i+k+1}\right)$ :

$$
\begin{aligned}
I I I & :-\sum_{\gamma, \alpha} G_{\alpha}^{i+1 *} v\left(g_{\alpha \delta}^{i+1}\right) \mu\left(G_{\beta}^{k} G_{\delta}^{i+2}\right) \circ d_{F}^{i} . \\
& =-\sum_{\gamma, \alpha, \delta} G_{\gamma}^{i *} g_{\gamma \alpha}^{i} v\left(g_{\alpha \delta}^{i+1}\right) \mu\left(G_{\beta}^{k} G_{\delta}^{i+2}\right) .
\end{aligned}
$$

Again the 3 cancel out. This completes the proof that $\mathcal{T}_{X}$ is a complex. Again, one can give a more abstract proof that $d^{2}(v \otimes G)=0$, based on (3. 1.13).

Note the fact that $\mathcal{T}_{X}$ is a complex, modulo knowing the same for $\mathcal{T}_{X / P}$, could equivalently be formulated as follows. Let

$$
J: F \cdot \otimes T_{P} \rightarrow T_{X / P}
$$

be defined by

$$
\begin{gathered}
J^{0}\left(e_{\alpha} \otimes v\right)= \\
\left(f_{\alpha} v, \sum_{i, \beta \gamma} v\left(g_{\beta \gamma}^{j}\right) G_{\beta}^{j *} \mu\left(e_{\alpha} G_{\gamma}^{j+1}\right)\right) \in T_{P} \oplus \mathcal{N}^{0}=\mathcal{T}_{X / P}^{0}, i=0
\end{gathered}
$$

$$
\begin{gathered}
J^{i}\left(G^{i} \otimes v\right)= \\
\left.\sum_{i, \beta \gamma} v\left(g_{\beta \gamma}^{j}\right) G_{\beta}^{j *} \mu\left(G^{i} G_{\gamma}^{j+1}\right)\right) \in \mathcal{N}^{i}=\mathcal{T}_{X / P}^{i}, i<0 .
\end{gathered}
$$


(cf. (3. 1.12)). Then $J$ is a map of complexes, whose mapping cone is $\mathcal{T}_{X}$. This what we have shown.

\subsubsection{Actions.}

Next, we will define an action of $\mathcal{T}_{X / P}$ on $I$. This action amounts to a pairing in the derived category

$$
\mathcal{T}_{X / P} \times F^{\cdot} \rightarrow F^{\cdot}
$$

that extends to a morphism

$$
\mathcal{T}_{X / P} \otimes F^{\cdot} \rightarrow F^{\cdot}
$$

(compatible with the differentials) . The pairing is defined as follows: the component

$$
\operatorname{Hom}\left(F^{i}, F_{+}^{j}\right) \otimes F^{i} \rightarrow F^{j}
$$

is the obvious map if $j \leq 0$ and 0 if $j=1$ (this is 'explained' by the remark that via the inclusion $\mathcal{N} \rightarrow \mathfrak{g l}\left(F_{+}\right)$, an element of $\mathcal{N}$ ' annihilates, hence preserves, the subcomplex $F_{+}^{1} \subset F_{+}$, hence induces an endomorphism of $F^{\cdot}=F_{+} / F_{+}^{1}$ ); the component

$$
T_{P} \otimes F^{i} \rightarrow F^{i}
$$

is defined by the postulation that it annihilate the standard basis $\left(G_{\beta}^{i}\right)$ of $F^{i}$, thus

$$
v \otimes \sum_{\beta} a_{\beta} G_{\beta}^{i} \mapsto \sum_{\beta} v\left(a_{\beta}\right) G_{\beta}^{i} .
$$

Now verifying that this yields a pairing of complexes amounts to commutativity, for all $j<0$, of the diagrams

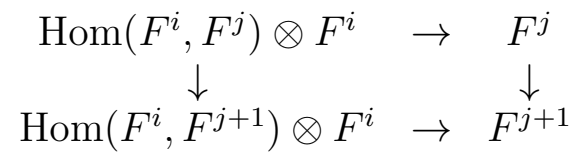

(which is obvious); and, for all $i<0$, of

(3. 2.18)

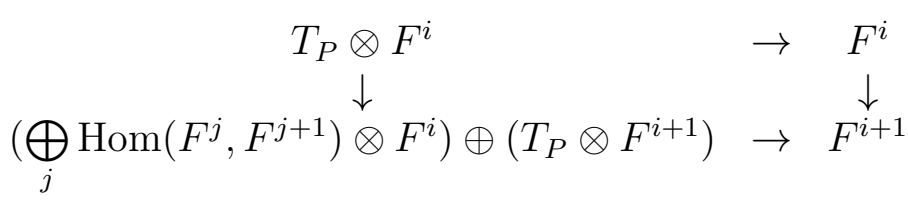

To check commutativity of (3. 2.18), consider a section

$$
s=v \otimes G_{\beta}^{i} \in T_{P} \otimes F^{i} .
$$

Then $s$ clearly goes to zero in $F^{i}$, hence in $F^{i+1}$ going clockwise. In $\bigoplus_{j} \operatorname{Hom}\left(F^{j}, F^{j+1}\right) \otimes$ $F^{i}$, the image of $s$ is

$$
-\sum_{j, \gamma, \alpha} G_{\gamma}^{i *} v\left(g_{\alpha \gamma}^{j}\right) G_{\gamma}^{j+1} \otimes G_{\beta}^{i}
$$


which maps to

$$
-\sum_{\alpha} v\left(g_{\alpha \beta}^{i}\right) G_{\alpha}^{i+1} \in F^{i+1}
$$

In $T_{P} \otimes F^{i+1}$, the image of $s$ is $v \otimes \sum_{\alpha} g_{\alpha \beta}^{i} G_{\alpha}^{i+1}$, which maps to

$$
\sum_{\alpha} v\left(g_{\alpha \beta}^{i}\right) G_{\alpha}^{i+1} \in F^{i+1} .
$$

The latter two add up to zero in $F^{i+1}$. Thus the image of $s$ going counterclockwise is zero as well. This shows the diagram commutes, hence we have a pairing of complexes as claimed.

Note that a similar recipe also defines an action of $\mathcal{T}_{X / P}$ on $F_{+} \sim A_{X}$, as well as on $A_{P}$ (the latter via the quotient $T_{P}$ of $\mathcal{T}_{X / P}$. Also, these actions are derivations with respect to the $A_{P}$-module structure on $F$ and $F_{+}$:

$$
\langle u, a b\rangle=a\langle u, b\rangle+\langle u, a\rangle b, a \in A_{P}, b \in F^{\cdot}, F_{+} .
$$

Indeed because the action of any $u \in \mathcal{N}$ is $A_{P}$-linear and such $u$ annihilates $A_{P}$, it suffices to check this for $u \in T_{P}, b=G_{\alpha}^{i}$, in which case it is immediate from the definition.

Note that it is not in general true that

$$
\text { (3. 2.20) } \quad\langle u, \mu(a b)\rangle \equiv \mu(\langle u, a\rangle b) \pm \mu(a\langle u, b\rangle) \bmod d F \text {. }
$$

where $d F$. is the image of the map $F \cdot[-1] \rightarrow F_{+}$induced by the differential $d$. For example, (3. 2.20) may fail if $u \in \mathcal{N}^{2}$. Nonetheless it is easy to see that (3. 2.20) holds if $u$ is of degree $\leq 1$. In particular, the induced pairing

$$
H^{0}\left(\mathcal{T}_{X / P}\right) \times A_{P} \rightarrow A_{X}
$$

is a derivation on $A_{P}$, hence the pairing

$$
H^{0}\left(\mathcal{T}_{X / P}\right) \times A_{X} \rightarrow A_{X}
$$

is a derivation on $A_{X}$. Note that because $F_{+}$has cohomology only in degree 1 and (3. 2.19) holds for $a, b \in F_{+}^{1}$, the diagram

$$
\begin{array}{ccccc}
\mathcal{T}_{X / P} \times F_{+} \times F_{+} & \stackrel{\text { id } \times \mu}{\rightarrow} & \mathcal{T}_{X / P} \times F_{+} & \\
\downarrow & & \downarrow & \langle,\rangle \\
\mathcal{T}_{X / P} \times F_{+} & \stackrel{\langle,\rangle}{\rightarrow} & F_{+} &
\end{array}
$$

commutes on cohomology.

Next, we define in a similar fashion an action

$$
\mathcal{T}_{X / P} \times\left(I \otimes T_{P}\right) \rightarrow I \otimes T_{P}
$$

via a pairing of complexes

(3. 2.23)

$$
\mathcal{T}_{X / P} \times\left(F \cdot \underset{31}{(F} T_{P}\right) \rightarrow F \cdot \otimes T_{P}
$$


Again, the key point is to define

$$
T_{P} \times\left(F^{i} \otimes T_{P}\right) \rightarrow F^{i} \otimes T_{P}
$$

by

$$
v \times \sum a G_{\alpha}^{i} \otimes w_{\alpha} \mapsto \sum_{\alpha}\left(a G_{\alpha}^{i} \otimes\left[v, w_{\alpha}\right]+v(a) G_{\alpha}^{i} \otimes w_{\alpha}\right), a \in A_{P}
$$

(note that this is compatible with our earlier definition setting $v\left(G_{\alpha}^{i}\right)=0$ ). The pairing

$$
\operatorname{Hom}\left(F^{i}, F^{j}\right) \times F^{i} \otimes T_{P} \rightarrow F^{j} \otimes T_{P}
$$

is the obvious one (acting on the $F^{i}$ factor only). The verification that this defines a pairing of complexes is again essentially obvious on the $\mathcal{N}$. subcomplex of $\mathcal{T}_{X / P}$, and it remains to check commutativity, for all $i<0$, of

$$
\begin{array}{ccc}
T_{P} \times F^{i} \otimes T_{P} & \downarrow & F^{i} \otimes T_{P} \\
\downarrow & & \downarrow \\
\left(\bigoplus_{j} \operatorname{Hom}\left(F^{j}, F^{j+1}\right) \otimes F^{i} \otimes T_{P}\right) \oplus\left(T_{P} \times F^{i+1} \otimes T_{P}\right) & \rightarrow & F^{i+1} \otimes T_{P}
\end{array}
$$

Indeed going clockwise, an element

$$
v \times G_{\alpha}^{i} \otimes w \mapsto \sum_{\beta} g_{\alpha \beta}^{i} G_{\beta}^{i+1}[v, w] .
$$

As for the counterclockwise direction, first going downwards,

$$
v \times G_{\alpha}^{i} \otimes w \mapsto\left(-\sum_{\gamma \beta} v\left(g_{\gamma \beta}^{i}\right) G_{\gamma}^{i *} \otimes G_{\beta}^{i+1} \times G_{\alpha}^{i} \otimes w, v \times \sum_{\beta} g_{\alpha \beta}^{i} G_{\beta}^{i+1} \otimes w\right)
$$

Then going rightwards, the first component maps to

$$
-\sum_{\beta} v\left(g_{\alpha \beta}^{i}\right) G_{\beta}^{i+1} \otimes w
$$

and the second to

$$
\sum_{\beta} g_{\alpha \beta}^{i} G_{\beta}^{i+1}[v, w]+\sum_{\beta} v\left(g_{\alpha \beta}^{i}\right) G_{\beta}^{i+1} \otimes w,
$$

and the two add up to (3. 2.25).

3.2.4. $\mathcal{T}_{X / P}$ is a dgla.

Next, we show that $\mathcal{T}_{X / P}$ itself admits a bracket, making it a dgla. This bracket is defined in the obvious way on element pairs within $\mathcal{N}_{X / P}$ or $T_{P}$. As for the cross terms $T_{P} \times \operatorname{Hom}\left(F^{j}, F_{+}^{i+j}\right)$, note first that $\left.\operatorname{Hom}\left(F^{j}, F^{i+j}\right)\right)$ has a standard basis of the form

(3. 2.26)

$$
\begin{aligned}
h_{\alpha \beta}^{-j, i+j}= & \left(G_{\alpha}^{j}\right)^{*} \otimes G_{\beta}^{i+j} \\
& 32
\end{aligned}
$$


(i.e. the rank-1 homomorphism taking the basis element $G_{\alpha}^{j}$ to $G_{\beta}^{i+j}$ ); with the convention that when $i+j=1,\left(G_{\beta}^{1}\right)$ is the unique element $1 \in F_{+}^{1}$. We define

$$
\left[v, h_{\alpha \beta}^{i j}\right]=0, \forall v \in T_{P}, \forall i, j, \alpha, \beta
$$

and extend by the derivation rule, i.e.

$$
\left[v, \sum_{\alpha \beta} c_{\alpha \beta} h_{\alpha \beta}^{i j}\right]=\sum_{\alpha \beta} v\left(c_{\alpha \beta}\right) h_{\alpha \beta}^{i j} .
$$

Now, the Jacobi identity for this bracket amounts to 2 identities:

$$
\begin{aligned}
{\left[\left[v_{1}, v_{2}\right], h\right] } & =\left[v_{1},\left[v_{2}, h\right]\right]-\left[v_{2},\left[v_{1}, h\right]\right], \\
{\left[v,\left[h_{1}, h_{2}\right]\right] } & =\left[\left[v, h_{1}\right], h_{2}\right]-\left[\left[v, h_{2}\right], h_{1}\right] .
\end{aligned}
$$

Indeed (3. 2.28) is obvious from the definition (both sides vanish on standard basis elements $h_{\alpha \beta}^{i j}$ ), while (3. 2.29) is obvious from the bilinearity of the bracket on $h_{1}, h_{2}$ and the derivation property of the action of $v$ on scalars (which again just amounts to the fact that both sides vanish on the standard basis). Thus we have a Lie bracket on $\mathcal{T}_{X / P}$.

To complete the proof that $\mathcal{T}_{X / P}$ is a dgla, it remains to verify the compatibility of the bracket with the differential $d$. Since this compatibility is already known within $\mathcal{N}$, we are reduced to showing

$$
\begin{aligned}
& d[v, h]=[d v, h]+[v, d h], v \in T_{P}, h=h_{\alpha \beta}^{i j}, \\
& d\left[v_{1}, v_{2}\right]=\left[d v_{1}, v_{2}\right]+\left[v_{1}, d v_{2}\right], v_{1}, v_{2} \in T_{P}
\end{aligned}
$$

where the LHS of (3. 2.30) is of course 0 by definition. Now we calculate, for $h=h_{\alpha \beta}^{-j, i+j}$ :

$$
[d v, h]=-\sum_{\gamma} v\left(g_{\beta \gamma}^{i+j}\right) G_{\alpha}^{j *} \otimes G_{\gamma}^{i+j+1}-(-1)^{i} \sum_{\delta} v\left(g_{\delta \alpha}^{j-1}\right) G_{\delta}^{j-1 *} G_{\beta}^{i+j},
$$

while

$$
\begin{gathered}
{[v, d h]=\left[v, \sum_{\gamma} g_{\beta \gamma}^{i+j} G_{\alpha}^{j *} \otimes G_{\gamma}^{i+j+1}+(-1)^{i} \sum_{\delta} g_{\delta \alpha}^{j-1} G_{\delta}^{j-1 *} G_{\beta}^{i+j}\right]} \\
=\sum_{\gamma} v\left(g_{\beta \gamma}^{i+j}\right) G_{\alpha}^{j *} \otimes G_{\gamma}^{i+j+1}+(-1)^{i} \sum_{\delta} v\left(g_{\delta \alpha}^{j-1}\right) G_{\delta}^{j-1 *} G_{\beta}^{i+j},
\end{gathered}
$$

which shows (3. 2.30). Then (3. 2.31) is equally easy:

$$
\begin{gathered}
{\left[v_{1}, d v_{2}\right]=\left[v_{1},-\sum_{\alpha \beta} v_{2}\left(g_{\alpha \beta}^{i}\right) G_{\alpha}^{i *} G_{\beta}^{i+1}\right]=-\sum_{\alpha \beta} v_{1}\left(v_{2}\left(g_{\alpha \beta}^{i}\right)\right) G_{\alpha}^{i *} G_{\beta}^{i+1},} \\
{\left[d v_{1}, v_{2}\right]=-\left[v_{2}, d v_{1}\right]=-\left[v_{2},-\sum_{\alpha \beta} v_{1}\left(g_{\alpha \beta}^{i}\right) G_{\alpha}^{i *} G_{\beta}^{i+1}\right]}
\end{gathered}
$$




$$
=\sum_{\alpha \beta} v_{2}\left(v_{1}\left(g_{\alpha \beta}^{i}\right)\right) G_{\alpha}^{i *} G_{\beta}^{i+1}
$$

and finally

$$
d\left[v_{1}, v_{2}\right]=-\sum_{\alpha \beta}\left[v_{1}, v_{2}\right]\left(g_{\alpha \beta}^{i}\right) G_{\alpha}^{i *} G_{\beta}^{i+1},
$$

which yields (3. 2.31). Thus we have shown that $\mathcal{T}_{P / X}$ is a dgla. Note that by construction, $\mathcal{N}$ is a dg Lie ideal in $\mathcal{T}_{X / P}$ and we have a dgla exact sequence

$$
0 \rightarrow \mathcal{N} \rightarrow \mathcal{T}_{P / X} \rightarrow T_{P} \rightarrow 0 .
$$

Next, we must check that the bracket just defined is compatible with the actions of $\mathcal{T}_{X / P}$ on $F$. and on $F \cdot \otimes T_{P}$ defined in the pervious paragraph, i.e. that

$$
\begin{array}{r}
\left\langle\left[u_{1}, u_{2}\right], a\right\rangle=\left\langle u_{1},\left\langle u_{2}, a\right\rangle\right\rangle-(-1)^{\operatorname{deg}\left(u_{1}\right) \operatorname{deg}\left(u_{2}\right)}\left\langle u_{2},\left\langle u_{1}, a\right\rangle\right\rangle, \\
\forall u_{1}, u_{2} \in \mathcal{T}_{X / P}, a \in F^{\cdot}, F \cdot \otimes T_{P} .
\end{array}
$$

Now in the case of the action on $F$, it is easy to see that we may assume first that $a=G_{\alpha}^{i}$, and second that $u_{1}, u_{2}$ are of the form $v$ or $h$ as above. Then in case $u_{1}$ or $u_{2}=v$, both sides of (3. 2.32) yield zero, while if both $u_{1}, u_{2} \in \mathcal{N} \subset \mathfrak{g l}\left(F_{\dot{+}}\right)$, (3. 2.32) holds by definition of the bracket on $\mathfrak{g l}$. The case $a=G_{\alpha}^{i}$ is similar.

Finally, we claim that the action of $\mathcal{T}_{X / P}$ in $F$-is compatible with the differential $d_{F}$, in other words, that $d_{\mathcal{T}_{X / P}}$ maps under the action to commutator with $d_{F}$, i.e

$$
\left\langle d_{\mathcal{T}_{X / P}}(u), a\right\rangle=d\langle u, a\rangle-(-1)^{\operatorname{deg} u}\left\langle u, d_{\mathcal{F} .}(a)\right\rangle, u \in \mathcal{T}_{X / P}, a \in F .
$$

But this is immediate when $u \in \mathcal{N}$, and when $u \in T_{P}$ it follows directly from (3. 1.11).

3.2.5. $\mathcal{T}_{X}$ is a dgla.

Since we know $\mathcal{T}_{X}$ is a complex and brackets between elements of $\mathcal{T}_{X / P}$ and $F \cdot \otimes T_{P}$ have been defined previously (cf. (3. 2.23)), defining a dgla structure on $\mathcal{T}_{X}$ now amounts to defining the bracket on terms of the form $F^{i} \otimes T_{P}$, and verifying compatibility and Jacobi identity. To this end, define

$$
\begin{aligned}
& {\left[a_{\alpha} G_{\alpha}^{i} v_{1}, a_{\beta} G_{\beta}^{j} v_{2}\right]=} \\
& \sum_{\gamma} a_{\alpha} v_{1}\left(a_{\beta}\right) \mu\left(G_{\alpha}^{i} G_{\beta}^{j}\right) v_{2}-\sum_{\delta} a_{\beta} v_{2}\left(a_{\alpha}\right) \mu\left(G_{\alpha}^{i} G_{\beta}^{j}\right) v_{1} \\
& +a_{\alpha} a_{\beta} \mu\left(G_{\alpha}^{i} G_{\beta}^{j}\right)\left[v_{1}, v_{2}\right] \\
& =\mu\left(G_{\alpha}^{i} G_{\beta}^{j}\right)\left[a_{\alpha} v_{1}, a_{\beta} v_{2}\right]
\end{aligned}
$$

To show $\mathcal{T}_{X}$ is a dgla we first check compatibility of the bracket with the differential , i.e.

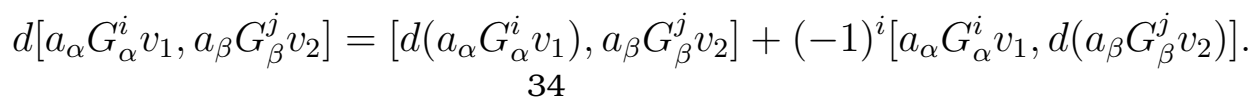


It is easy to see that we may assume $a_{\alpha}=a_{\beta}=1$. In this case the LHS of (3. 2.34) is just

$$
\begin{gathered}
d \mu\left(G_{\alpha}^{i} G_{\beta}^{j}\right)\left[v_{1}, v_{2}\right]+\mu\left(\mu\left(G_{\alpha}^{i} G_{\beta}^{j}\right) d\left[v_{1}, v_{2}\right]\right)= \\
\mu\left(d G_{\alpha}^{i} G_{\beta}^{j}\right)\left[v_{1}, v_{2}\right]-(-1)^{i} \mu\left(G_{\alpha}^{i} d G_{\beta}^{j}\right)\left[v_{1}, v_{2}\right]+\mu\left(\mu\left(G_{\alpha}^{i} G_{\beta}^{j}\right) d\left[v_{1}, v_{2}\right]\right)
\end{gathered}
$$

On the other hand the first bracket of the RHS yields

$$
\begin{gathered}
{\left[d\left(G_{\alpha}^{i}\right) v_{1}+\mu\left(G_{\alpha}^{i} d v_{1}\right), G_{\beta}^{j} v_{2}\right]=} \\
\sum_{\gamma} v_{2}\left(g_{\alpha \gamma}^{i}\right) \mu\left(G_{\gamma}^{i+1} G_{\beta}^{j}\right) v_{1}+\mu\left(d G_{\alpha}^{i} G_{\beta}^{j}\right)\left[v_{1}, v_{2}\right]+ \\
\sum_{\delta} v_{1}\left(g_{\beta \delta}^{j}\right) \mu\left(G_{\alpha}^{i} G_{\delta}^{j+1}\right) v_{2}+\mu\left(\mu\left(G_{\alpha}^{i} G_{\beta}^{j}\right)\left[d v_{1}, v_{2}\right]\right)
\end{gathered}
$$

while the second bracket yields

$$
\begin{gathered}
{\left[G_{\alpha}^{i} v_{1}, d\left(G_{\beta}^{j}\right) v_{2}+\mu\left(G_{\beta}^{j} d v_{2}\right)\right]=} \\
(-1)^{i+1} \sum_{\delta} v_{1}\left(g_{\beta \delta}^{j}\right) \mu\left(G_{\alpha}^{i} G_{\delta}^{j+1}\right) v_{2}+\mu\left(G_{\alpha}^{i} d G_{\beta}^{j}\right)\left[v_{1}, v_{2}\right]+ \\
\left.(-1)^{i+1} \sum_{\gamma} v_{2}\left(g_{\alpha \gamma}^{i}\right) \mu\left(G_{\beta}^{j} G_{\gamma}^{i+1}\right) v_{1}+(-1)^{i} \mu\left(\mu\left(G_{\alpha}^{i} G_{\beta}^{j}\right)\left[v_{1}, d v_{2}\right]\right)\right)
\end{gathered}
$$

Therefore (3. 2.34) holds.

It remains to verify the Jacobi identity, in the form

$$
\begin{array}{r}
{\left[\left[G_{\alpha}^{i} v_{1}, G_{\beta}^{j} v_{2}\right], G_{\gamma}^{k} v_{3}\right]=} \\
{\left[G_{\alpha}^{i} v_{1},\left[G_{\beta}^{j} v_{2}, G_{\gamma}^{k} v_{3}\right]\right]-(-1)^{(i+1)(j+1)}\left[G_{\beta}^{j} v_{2},\left[G_{\alpha}^{i} v_{1}, G_{\gamma}^{k} v_{3}\right]\right]}
\end{array}
$$

As remarked earlier, we may assume that $\mu\left(G_{\alpha}^{i} G_{\beta}^{j}\right)$ is a standard basis element of $F^{i+j-1}$, of the form $G_{\delta}^{i+j-1}$ for some $\delta$, and similarly for other $\mu$ products. In this case, (3. 2.35) follows directly from the definition (3. 2.33). This finally completes the proof the $\mathcal{T}_{X}$ is a dgla.

As for the extension of the action of $\mathcal{T}_{X / P}$ on $F_{+}$to an action of $\mathcal{T}_{X}$, this is defined by setting

$$
\left\langle G_{\alpha}^{i} v, a G_{\beta}^{j}\right\rangle=v(a) \mu\left(G_{\alpha}^{i} G_{\beta}^{j}\right), i \leq 0, j \leq 1
$$

(note that $G_{\alpha}^{i} \otimes v$ has degree $i-1$ in $\mathcal{T}_{X}$, so both sides have the same degree, viz. $i+j-1$, as required). The same formula also defines an action

$$
\mathcal{T}_{X} \times F_{+} \rightarrow F_{\dot{+}} .
$$

It is easy to check that this is a pairing of complexes, and that it is compatible with the bracket on $\mathcal{T}_{X}$, and that all elements of degree $\leq 1$ in $\mathcal{T}_{X}$ acts on $F_{+}$as derivations $\bmod$ the image of $F$. Therefore, we get a derivation action

$$
H^{0}\left(\mathcal{T}_{X}\right) \times A_{X} \rightarrow A_{X}
$$

which by its very construction is compatible with that of $T_{P}$ on $A_{P}$ and $\mathcal{T}_{X / P}$ on $I$. 
We claim next that $\mathcal{T}_{X}$ is an $A_{X}$-module, in the sense that there is (in the derived category) a multiplication pairing

$$
A_{X} \times \mathcal{T}_{X} \rightarrow \mathcal{T}_{X},
$$

, or to be precise, a map of complexes with appropriate 'action' properties

$$
F_{+} \times \mathcal{T}_{X} \rightarrow \mathcal{T}_{X}[-1] .
$$

This is simply defined by postmultiplication:

$$
\begin{aligned}
\left\langle G_{\alpha}^{i}, G_{\beta}^{j} \otimes v\right\rangle & =\mu\left(G_{\alpha}^{i} G_{\beta}^{j}\right) \otimes v, \\
\left\langle G_{\alpha}^{i}, G_{\beta}^{j} \otimes G_{\gamma}^{k *}\right\rangle & =\mu\left(G_{\alpha}^{i} G_{\beta}^{j}\right) \otimes G_{\gamma}^{k *} .
\end{aligned}
$$

Note that the $A_{X}$-module structure (3. 2.38) shows that the cohomology sheaves of $\mathcal{T}_{X}$ are $A_{X}$-modules compatibly with their $A_{P}$-module structure, hence clearly $A_{X}$-coherent. In particular $\mathcal{T}_{X}$ is equivalent to its sheaf-theoretic restriction on $X$. Since $\mathcal{T}_{X}$ is a bounded complex with $A_{X}$-coherent cohomology, it defines an element of the bounded, coherent derived category $D_{c}^{b}(X)$.

We note that even though in the above construction we used the 'full dgla' model for the normal atom $\mathcal{N}_{X / P}$, leading to a dgla model for $\mathcal{T}_{X}$, we could instead have used the reduced model $\mathcal{N}^{\text {red }}$ (see $\S 2.7$ ) for $\mathcal{N}$, thus leading to a Lie atom $\mathcal{T}_{X}^{\text {red }}$ weakly equivalent to $\mathcal{T}_{X}$.

3.2.6. Dependence on choices.

The above construction of the tangent atom $\mathcal{T}_{X}$ of an affine scheme $X$ depended, in addition to a choice of affine embedding $X \rightarrow P$, also on a choice of free resolution of $I=I_{X / P}$. We claim next that, still fixing the embedding $X \rightarrow P$, the Lie atom $\mathcal{T}_{X}$ is independent of the resolution up to Lie quasi-isomorphism, i.e. a composition of Lie atom homomorphisms inducing an isomorphism on cohomology and their inverses. This is just a trivial variation of the usual statement on independence of resolution. The precise statement is as follows:

Lemma 3.3. . Let ${ }^{1} F^{\cdot},{ }^{2} F$ be free resolutions of the ideal of the closed subscheme $X \rightarrow P$ and ${ }^{1} \mathcal{T}_{X},{ }^{2} \mathcal{T}_{X}$ the associated Lie atom. Then there exists a third such resolution ${ }^{3} F$. with associated atom ${ }^{3} \mathcal{T}_{X}$, together with direct Lie quasi-isomorphisms to the associated Lie atom

$$
{ }^{1} \mathcal{T}_{X} \rightarrow{ }^{3} \mathcal{T}_{X} \leftarrow{ }^{2} \mathcal{T}_{X}
$$

Proof. As is well known, there exists a resolution ${ }^{3} F$ of $I$ together with maps

$$
{ }^{i} p::^{3} F \cdot \rightarrow{ }^{i} F \cdot{ }^{i} q:^{i} F \cdot \rightarrow{ }^{3} F .
$$

such that ${ }^{i} p^{i} q$ is the identity and ${ }^{i} q^{i} p$ is homotopic to the identity for $i=1,2$. Moreover we may assume ${ }^{i} q$ takes a standard generator $G_{\alpha}^{j}$ to a standard generator. Then we get maps

$$
{ }^{i} \mathcal{N}=\operatorname{Hom}\left({ }^{i} F \cdot{ }^{i} F_{+}\right) \rightarrow{ }^{3} \mathcal{N}, i=1,2,
$$




$$
h \mapsto^{i} q \circ h \circ^{i} p .
$$

These are clearly Lie quasi-isomorphisms. By construction, these extend to maps as in (3. 2.40).

Next we take up the question of dependence of $\mathcal{T}_{X}$ on the affine embedding $X \rightarrow P$. To make this dependence explicit, we will henceforth denote by $\mathcal{T}_{X}(P)$ an explicit representative of the Lie atom denoted formerly by $\mathcal{T}_{X}$, based on a choice of resolution $F$, and uniquely determined up to Lie quasi-isomorphism. What we claim is that the weak equivalence class of $\mathcal{T}_{X}(P)$ is independent of the embedding; more precisely, given two embeddings $X \rightarrow P, X \rightarrow Q$, there is a Lie atom $\mathcal{T}$ and (direct) weak equivalences

$$
\mathcal{T}_{X}(P) \rightarrow \mathcal{T}, \mathcal{T}_{X}(Q) \rightarrow \mathcal{T}
$$

(we recall that a weak equivalence is not necessarily invertible even in the derived category; a direct weak equivalence is one given by a morphism of complexes). In fact, we claim that can take

$$
\mathcal{T}=\mathcal{T}_{X}(P \times Q)
$$

where $X \rightarrow P \times Q$ embeds via the diagonal $\Delta_{X} \subset X \times X$. What we need to do then is construct each of the weak equivalences (3. 2.41).

To this end set $R=P \times Q$. Note that the embedding $X \rightarrow R$ lifts to $P \rightarrow R$, which is a cross section of projection $R \rightarrow P$. We first contrcut a Lie homomorphism

$$
\mathcal{T}_{X}(P) \rightarrow \mathcal{T}_{X}(R)
$$

This is done as follows. Let $F_{P}$ be a free resolution of $I_{X / P}$ Then we can construct a free resolution $F_{R}$ of $I_{X / R}$ such that each $F_{R}^{i}$ contains $F_{P}^{i} \otimes A_{R}$ as a direct summand. In fact, since the embedding $P \rightarrow R$ is automatically regular, we may assume that

$$
F_{R}^{i}=F_{P}^{i} \otimes A_{R} \oplus F_{Q}^{i}
$$

where $F_{\dot{Q}}$ is a Koszul resolution of $I_{P / R}$. Set

$$
S \cdot=\mathcal{T}_{X}(P) \otimes A_{R}
$$

which has an obvious structure of Lie atom. Then as in the proof of Lemma 3.3 above, we get a Lie homomorphism

$$
\mathcal{N}_{X / P} \otimes A_{R}=\operatorname{Hom}_{R}\left(F_{P} \otimes A_{R}, F_{P+} \otimes A_{R}\right) \rightarrow \mathcal{N}_{X / R}=\operatorname{Hom} \cdot\left(F_{R}, F_{R+}\right)
$$

which extends to a Lie homomorphism $S \rightarrow \mathcal{T}_{X}(R)$. Combined with the obvious homomorphism $\mathcal{T}_{X}(P) \rightarrow S$, this gives the desired homomorphism (3. 2.43). Note 
that this homomorphism can be represented schematically as follows

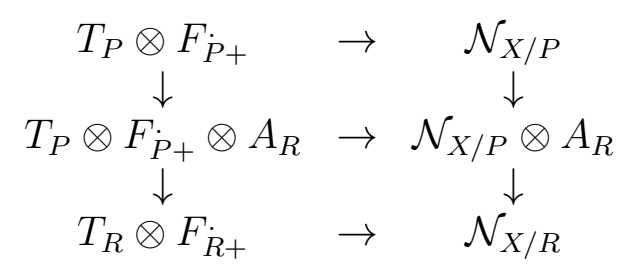

Note that since $F_{P} \otimes A_{R}$ is a resolution of $I_{X \times Q \rightarrow P \times Q}$, we can represent the above diagram (3. 2.44) as follows

(3. 2.45)

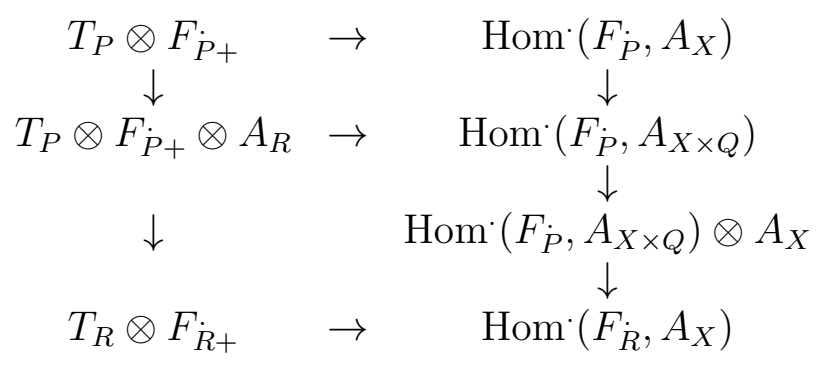

Now the composite of the left arrows is clearly an injection with cokernel $T_{Q} \otimes A_{X}$, while the composite of the 2 upper right arrows is an isomorphism. On the other hand as in $\S 2.7$ we have that $\operatorname{Hom}\left(F_{\dot{Q}}, A_{X}\right)=\mathcal{N}_{P / R} \otimes A_{X}$ is weakly equivalent to the restricted reduced normal atom

$$
\mathcal{N}_{Q / R}^{\mathrm{red}} \otimes A_{X}=N_{P / R}[-1] \otimes A_{X} \simeq T_{Q}[-1] \otimes A_{X} .
$$

Similarly, we have an exact sequence

$$
\mathcal{N}_{X / P}^{\mathrm{red}} \rightarrow \mathcal{N}_{X / R}^{\mathrm{red}} \rightarrow T_{Q}[-1] \otimes A_{X} \rightarrow
$$

which means that, up to weak equivalence, the cokernel of the bottom right arrow is $T_{Q} \otimes A_{X}$. Therefore the map from the top row of (3. 2.45) is a weak equivalence, as claimed.

\subsubsection{Functoriality.}

We claim next, still in the embedded case, that a morphism

(3. 2.46)

$$
f: X \rightarrow Y
$$

of affine schemes induces a map

$$
\text { df }: \mathcal{T}_{X} \rightarrow f^{*} \mathcal{T}_{Y} .
$$

A precise formulation is as follows. First, note that given affine embeddings

$$
X \rightarrow P, Y \rightarrow Q,
$$

a morphism $f$ as in (3. 2.46) extends to a morphism $f: P \rightarrow Q$. Moreover since $\mathcal{T}_{Y}(Q)$ is a complex of finite free $A_{Q}$-modules, $f^{*} \mathcal{T}_{Y}=f^{!} \mathcal{T}_{Y}$ refers unambiguously to the termwise pullback, which is, in fact, a Lie atom (cf. [11]). 
Then the precise assertion is that there exists another weak-equivalence representatives $\mathcal{T}_{X}\left(P_{1}\right)$ of $\mathcal{T}_{X}(P)$, an extension of $f$ to a morphism $\tilde{f}: P_{1} \rightarrow Q$ with a map of Lie atoms

$$
d f: \mathcal{T}_{X}\left(P_{1}\right) \rightarrow \tilde{f}^{*} \mathcal{T}_{Y}(Q)
$$

(here we mean an 'actual' map of complexes, without inverting any weak equivalences). Moreover $\tilde{f}^{*} \mathcal{T}_{Y}(Q)$ admits a structure of a $\mathcal{T}_{X}$-module such that $d f$ is $\mathcal{T}_{X}$-linear. Note that this would make the mapping cone of (3. 2.48) itself into a Lie atom, which (compare [11]) may be denoted $\mathcal{N}_{f / Y}[-1]$ ).

Now the construction of (3. 2.48) is the following. Identifying $X$ with the graph of $f$, we have a diagram

$$
\begin{array}{ccc}
X \rightarrow & P \times Y \rightarrow \\
\searrow & \downarrow \\
& P \times Q \rightarrow & \\
& & \\
& &
\end{array}
$$

which induces a diagram

$$
\begin{array}{ccc}
T_{P \times Q} \otimes A_{X} & \rightarrow & \mathcal{N}_{X / P \times Q} \\
\downarrow & \downarrow & \downarrow \\
T_{Q} \otimes A_{X} & \rightarrow & f^{*} \mathcal{N}_{Y / Q}
\end{array}
$$

where the right vertical arrow comes from the fact that a resolution of $Y \rightarrow Q$ pulls back to a resolution of $P \times Y \rightarrow P \times Q$, hence can be assumed so be a subcomplex, and termwise a direct summand, of a resolution of $X \rightarrow P \times Q$. Then (3. 2.50) yields a map as in (3. 2.48) with $P_{1}=P \times Q$, and it is straightforward to check that this map is indeed $A_{X}$-linear.

Finally, we claim that the 'functoriality map' $d f$ is indeed functorial, in the sense that given morphisms of affine schemes

$$
X \stackrel{f}{\rightarrow} Y \stackrel{g}{\rightarrow} Z
$$

then commutes

$$
\begin{array}{ccc}
\mathcal{T}_{X} & \stackrel{d f}{\longrightarrow} & f^{*} \mathcal{T}_{Y} \\
\searrow & d(g \circ f) & \downarrow f^{*} d g \\
& & f^{*} g^{*} \mathcal{T}_{Z} .
\end{array}
$$

This can be made precise (via explicit representatives), and proven very similarly to the above. This finally completes the proof of Theorem 3.2.

Corollary 3.4. If $X$ is a smooth affine variety, then the weak equivalence class of $\mathcal{T}_{X}$ coincides with that of the usual tangent module $T_{X}=\operatorname{Der}\left(A_{X}\right)$. 
Proof. Any affine embedding $X \rightarrow P$ is regular, so we may use for the normal atom $\mathcal{N}_{X / P}$ the shifted normal module $N_{X / P}[-1]$. In this case $\mathcal{T}_{X}$ may be identified with the kernel of the surjection

$$
T_{P} \otimes A_{X} \rightarrow N_{X / P}
$$

which, as is well known, is just $T_{X}$.

3.3. Sheafification. Let $\mathcal{T}_{X}(P)$ be the termwise sheafification of $\mathcal{T}_{X}(P)$, as complex of sheaves of (free) $\mathcal{O}_{P}$-modules on $P$. Clearly, $\mathcal{T}_{X}(P)$ is acyclic off $X$, hence is quasi-isomorphic to its (topological) restriction on $X$, i.e. the natural map

$$
\mathcal{T}_{X}(P)^{\sim} \rightarrow i_{X *} i_{X}^{-1} \mathcal{T}_{X}(P)
$$

is a quasi isomorphism. Hence, we may consider $\mathcal{T}_{X}(P)^{\sim}$ as a complex of sheaves on $X$ (though the sheaves are not $\mathcal{O}_{X}$-modules). We have

$$
\mathcal{T}_{X / P}=\Gamma\left(P, \mathcal{T}_{X}(P) \tilde{)} \sim \Gamma\left(X, i_{X}^{-1} \mathcal{T}_{X}(P)\right)\right.
$$

As a special case of functoriality, consider a function $0 \neq f \in A_{P}$ and the associated principal affine open $X_{f} \stackrel{i}{\hookrightarrow} X$, which fits in a diagram

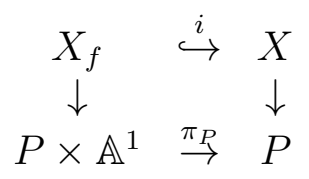

Then it is easy to see that the functoriality map

$$
\mathcal{T}_{X_{f}}\left(P \times \mathbb{A}^{1}\right) \rightarrow i ! \mathcal{T}_{X}(P)
$$

is a quasi-isomorphism, as is the natural map

$$
\mathcal{T}_{X_{f}}\left(P \times \mathbb{A}^{1}\right) \rightarrow \mathcal{T}_{X}(P)_{f}
$$

where the latter refers to termwise localization.

\subsection{Maps of affine schemes: tangent dgla. Let}

$$
f: X \rightarrow Y
$$

be a mapping of affine schemes. It is natural to consider tangent data to $f$, that is, a compatible set of tangent data to $X, Y$ and $f$. This can be done as follows.

Given affine embeddings $X \rightarrow P, Y \rightarrow Q, f$ can be extended to a map $P \rightarrow Q$. Replacing $X \rightarrow P$ by the graph embedding $X \rightarrow P \times Q$, we may assume $P \rightarrow Q$ is a product projection. Then we have an injection $I_{Y, Q} \rightarrow I_{X, P}$ which extends to the free resolutions $F_{\dot{Y}} \rightarrow F_{\dot{X}}$, and we may moreover assume that each $F_{Y}^{i} \otimes A_{P} \rightarrow F_{X}^{i}$ is a direct summand inclusion. We can identify the functor $f^{!}$on complexes with $f^{!} \cdot=\cdot \otimes_{A_{Y}} F_{\dot{X}+}$. Then the complex $f^{!}\left(\mathcal{N}_{Y / Q}\right)$ can be represented by

$$
\mathcal{H o m}_{A_{Q}}\left(F_{\dot{Y}}, F_{+X}\right)=\underset{40}{\mathcal{H} o m_{A_{P}}}\left(F_{\dot{Y}} \otimes A_{P}, F_{+X}\right)
$$


and there are maps

$$
\mathcal{N}_{X / P} \rightarrow f^{!}\left(\mathcal{N}_{Y / Q}\right) \leftarrow \mathcal{N}_{Y / Q}
$$

The mapping cone of (3. 4.54) can be represented by the sub-dgla of $\mathcal{N}_{X / P} \oplus \mathcal{N}_{Y / Q}$ consisting of pairs $(a, b)$ such that $a$ vanishes on the subcomplex $F_{\dot{Y}} \otimes A_{P} \subset F_{\dot{X}}$. We denote this mapping cone by $\mathcal{N}_{f}$ or, more properly, $\mathcal{N}_{f, P, Q}$, and refer to it as the normal dgla of $f$.

Next, proceeding as in the case of schemes, we can construct a suitable representative of the mapping cone $K$ of

$$
T_{P} \otimes F_{+X} \rightarrow T_{Q} \otimes F_{+X} \leftarrow T_{Q} \otimes F_{+Y},
$$

together with a map of $K$ to $\mathcal{N}_{f}$, so that the mapping cone of $K \rightarrow \mathcal{N}_{f}$ is a dgla, called the tangent dgla to $f$ and denoted $\mathcal{T}_{f}$ or more properly, $\mathcal{T}_{f}(P, Q)$. By construction, $\mathcal{T}_{f}(P, Q)$ is the mapping cone of

$$
\mathcal{T}_{X}(P) \oplus \mathcal{T}_{Y}(Q) \rightarrow f^{!} \mathcal{T}_{Y}(Q) .
$$

3.5. Reduced tangent algebra. As was the case for the normal algebra, the tangent algebra $\mathcal{T}_{X}(P)$ admits a reduced, weakly equivalent version ${ }^{\text {red }} \mathcal{T}_{X}(P)$ which is convenient for applications because it has a smaller $H^{2}$ (in fact, the $H^{2}$ will vanish when $X$ is a locally complete intersection, which is not the case for $\mathcal{T}_{X}$ itself). This dgla is closely related to, essentially a dual version of, the GrothendieckLichtenbaum-Schlessinger cotangent complex of $X$ (cf. [6]).

Theorem 3.5. Given an affine embedding $X \rightarrow P$, there is a dgla ${ }^{\text {red }} \mathcal{T}_{X}(P)$, together with a direct weak equivalence

$$
{ }^{\mathrm{red}} \mathcal{T}_{X}(P) \rightarrow \mathcal{T}_{X}(P)
$$

such that for all $i>1, H^{i}\left({ }^{\operatorname{red}} \mathcal{T}_{X}(P)\right)$ is supported on the locus of non-lci points of $X$. Moreover, the dgla quasi-isomorphism class of ${ }^{\operatorname{red}} \mathcal{T}_{X}(P)$ depends only on the isomorphism class of $X$ as scheme over $\mathbb{C}$.

Proof. Recall that the reduced normal algebra ${ }^{\text {red }} \mathcal{N}={ }^{\text {red }} \mathcal{N}_{X / P}$ admits an injective dgla map

$$
\lambda:{ }^{\operatorname{red}} \mathcal{N} \rightarrow \mathcal{N}^{\prime} \stackrel{\text { id }+\pi}{\longrightarrow} \mathcal{N}
$$

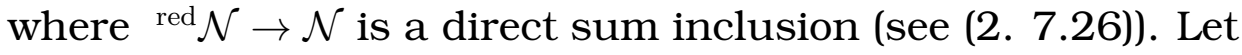

$$
\rho: \mathcal{N}^{\mathrm{id}-\pi} \mathcal{N}^{\prime} \rightarrow{ }^{\mathrm{red}} \mathcal{N}
$$

be the obvious left inverse to $\lambda$ (where $\mathcal{N}^{\prime} \rightarrow{ }^{\text {red }} \mathcal{N}$ is the projection). Recall (see (3. 2.15)) that $\mathcal{T}_{X}(P)$ could be defined as a mapping cone of

$$
J: T_{P} \otimes F_{+} \rightarrow \mathcal{N} .
$$

Now by the definition of $J$, we have, for $v \otimes G \in T_{P} \otimes F_{+}^{i}$,

$$
J(v \otimes G)\left(e_{\alpha}\right)=v\left(f_{\alpha}\right) G,
$$




$$
J(v \otimes G)\left(s_{\alpha \beta}\right)=v\left(f_{\alpha}\right) \mu\left(e_{\beta} \otimes G\right) .
$$

Therefore the component of $J(v \otimes G)$ in $\operatorname{Hom}\left(F_{0}^{-1}, F^{0}\right)$ is equal to $\pi$ of its component in $\operatorname{Hom}\left(F^{0}, A_{P}\right)$, i.e.

$$
\left.J(v \otimes G)\right|_{F_{0}^{-1}}=\pi\left(\left.J(v \otimes G)\right|_{F^{0}}\right) .
$$

Thus it follows that the image of $J$ is contained in that of id $+\pi$, hence $J$ factors through a map

i.e. $J=\lambda \circ{ }^{\mathrm{red}} J$. Set

$$
{ }^{\mathrm{red}} J=\rho \circ J: T_{P} \otimes F_{+} \rightarrow{ }^{\mathrm{red}} \mathcal{N},
$$

$$
{ }^{\mathrm{red}} \mathcal{T}_{X}(P)=\text { mapping cone }\left({ }^{\mathrm{red}} J\right) .
$$

Then the fact that ${ }^{\mathrm{red}} \mathcal{N} \rightarrow \mathcal{N}$ is an injective Lie homomorphism and a weq and that $\mathcal{T}_{X}$ is a dgla implies that ${ }^{\operatorname{red}} \mathcal{T}_{X}(P)$ is a dgla, weakley equivalent to $\mathcal{T}_{X}$.

Now to complete the proof, it remains to prove the lat statement, i.e. independence of choices. To this end, we modify the argument of subsection 3.2.6 as follows, using notation as there. Choosing standard resolutions of $X \rightarrow P, X \rightarrow R$, we obtain such for $X \rightarrow R$, whence compatible complexes $F_{i, P}, F_{i, R}$ etc. and then we have dgla homomorphisms

$$
{ }^{\text {red }} \mathcal{N}_{X / P} \rightarrow{ }^{\text {red }} \mathcal{N}_{X / P} \otimes A_{R} \rightarrow{ }^{\operatorname{red}} \mathcal{N}_{X / R} .
$$

Then we have a commutative diagram

$$
\begin{array}{ccc}
T_{P} \otimes F_{P+} & \rightarrow & \operatorname{red} \mathcal{N}_{X / P} \\
\downarrow & & \downarrow \\
T_{R} \otimes F_{R+} & \rightarrow & \operatorname{red} \mathcal{N}_{X / R}
\end{array}
$$

where the left column (as mapping cone) is clearly naturally quasi-isomorphic to $T_{Q} \otimes A_{X}[-1]$. On the other hand the discussion in subsection 3.2.6, together with Corollary 2.9 show that the same is true of the right column. Thus, the horizontal maps together give a dgla quasi-isomorphism

$$
{ }^{\text {red }} \mathcal{T}_{X}(P) \rightarrow{ }^{\text {red }} \mathcal{T}_{X}(R) .
$$

This yields our conclusion.

Since $\mathcal{T}_{X}$ is defined to be the weak equivalence class of $\mathcal{T}_{X}(P)$, we may take ${ }^{r e d} \mathcal{T}_{X}(P)$ as a representative of $\mathcal{T}_{X}$; we call representatives obtained in this way reduced.

3.6. Comparison. The complex $\mathcal{T}_{X}$ is a bit complicated, therefore in applications it is important to be able to compare it to something simpler, like a module or the Ext-dual of a module. The following result, based on Ischebeck's Theorem (see [2] or [7], p. 104) is useful in this connection.

First a definition. For any algebraic scheme $X$, we denote by $\mathrm{NCI}_{X} \subset X$ the (closed) locus of point $x \in X$ where $X$ is not locally a complete intersection. 
Theorem 3.6. Let $X \rightarrow P$ be an affine scheme.Then

(i) there is a natural map

$$
\tau_{X}: \operatorname{Hom}\left(\Omega_{X}, A_{X}\right) \rightarrow{ }^{\operatorname{red}} \mathcal{T}_{X}(P) ;
$$

(ii) the induced map on cohomology

$$
H^{i}\left(\tau_{X}\right): \operatorname{Ext}^{i}\left(\Omega_{X}, A_{X}\right) \rightarrow H^{i}\left({ }^{\operatorname{red}} \mathcal{T}_{X}(P)\right)
$$

is always bijective for $i=0$ and injective for $i=1$; moreover, $H^{1}\left(\tau_{X}\right)$ is bijective and $H^{2}\left(\tau_{X}\right)$ is injective provided $X$ is reduced (more generally, a generic locally complete intersection with no embedded points);

(iii) $\tau_{X}$ induces an isomorphism on cohomology in degrees $\leq k$ provided, for all $x \in X$, that

(3. 6.60)

$$
\operatorname{depth}_{x} A_{X}-\operatorname{dim}_{x} \mathrm{NCI}_{X} \geq k .
$$

(iv) In particular, if $X$ is Cohen-Macaulay and lci in codimension 1, then $\tau_{X}$ is a cohomological isomorphism in degrees $\leq 2$, i.e.

$$
H^{i}\left(\tau_{X}\right): \operatorname{Ext}_{X}^{i}\left(\Omega_{X}, A_{X}\right) \simeq H^{i}\left({ }^{\operatorname{red}} \mathcal{T}_{X}(P)\right), i \leq 2 .
$$

Proof. Consider the following complex in degrees $\leq 1$ :

$$
G^{\cdot}: \quad \ldots \rightarrow F_{1}^{-1} \otimes A_{X} \rightarrow F^{0} \otimes A_{X} \rightarrow \Omega_{P} \otimes A_{X} .
$$

This is a complex of free $A_{X}$-modules, whose cohomology in degree 1 is clearly $\Omega_{X}$, whence a morphism

$$
\check{\tau}_{X}: G \rightarrow \Omega_{X} .
$$

Locally at any lci point $x \notin \mathrm{NCI}_{X}$, the resolution $F$. is equivalent to a Koszul resolution, and therefore the complex $G$ is exact at $x$ in degrees $<1$. Thus for $i<1, H^{i}(G)$ is supported on $\mathrm{NCI}_{X}$. Now the complex $\operatorname{Hom}_{X}\left(G^{\prime}, A_{X}\right)$ is quasiisomorphic to ${ }^{\text {red }} \mathcal{T}_{X}(P)[1]$, therefore by dualizing $\check{\tau}_{X}$ we get $\tau_{X}$. Moreover we have a standard spectral sequence

$$
E_{2}^{p . q}=\operatorname{Ext}_{X}^{p}\left(H^{q}(G), A_{X}\right) \Rightarrow H^{p+q+1}\left({ }^{\operatorname{red}} \mathcal{T}_{X}(P)\right) .
$$

From this spectral sequence the assertions of (ii) follow easily; for example the kernel of $H^{2}\left(\tau_{X}\right)$ comes from $\operatorname{Ext}^{0}\left(H^{-1}(G), A_{X}\right)$, hence vanishes provided the support of $H^{-1}(G)$, i.e. $\mathrm{NCI}_{X}$, is a proper subscheme and $X$ has no embedded points.

Finally for part (iii) we use Ischebeck's Theorem which says that the $\operatorname{Ext}^{p}$ vanishes provided $q<1$ and

$$
p<\operatorname{depth} \mathrm{A}_{\mathrm{X}}-\operatorname{dim} \mathrm{NCI}_{X} .
$$

Therefore $\tau_{X}$ is an isomorphism in the range as claimed.

Note that the condition of part (iii) of the theorem applies whenever $X$ is either normal and Cohen-Macaulay, or a locally complete intersection curve. For deformation theory, it is the cohomology in degrees $\leq 2$ of $\mathcal{T}_{X}$ that matters. 
3.7. Example: a limit curve. We consider an example extending ones which occur in the local study of limits of covering maps of curves ([3], extending earlier work by Beauville) and families of nodal plane curves (Severi varieties)[8]. Let $C \subset \mathbb{A}_{u, v}^{2}$ be the curve with equation $u v$. It has components $C_{1}=\mathbb{A}_{u}^{1}, C_{2}=\mathbb{A}_{v}^{1}$. Its tangent algebra is

(3. 7.62)

$$
\mathcal{T}_{C}: T_{\mathbb{A}^{2}} \otimes F_{C, \mathbb{A}^{2},+} \rightarrow \mathcal{N}_{C}=\operatorname{Hom}\left(F_{C, \mathbb{A}^{2}}, F_{C, \mathbb{A}^{2},+}\right),
$$

and may be written symbolically as

(3. 7.63)

$$
A_{C}<\partial_{v}, \partial_{u}>\stackrel{u v}{\rightarrow} A_{C},
$$

or more simply as

(3. 7.64)

$$
2 A_{C} \stackrel{(u, v)}{\longrightarrow} A_{C} .
$$

Note that a basis for $\mathcal{N}_{C}$ is given by $\delta_{C}=[u v]^{*}$, i.e. the map that takes the value 1 on the resolution element $[u v]$ corresponding to the ideal generator $u v$.

Now let $X \subset \mathbb{A}_{x, y, z}^{n+2}=: Q, n \geq 0$ be the subscheme with equation $x y$, i.e. a copy of $C \times \mathbb{A}^{n}$, with components $X_{1}=\mathbb{A}_{x, \underline{z}}^{n+1}, X_{2}=\mathbb{A}_{y, \underline{z}}^{n+1}$. The tangent algebra $\mathcal{T}_{X}$ is similarly given symbolically by the complex

$$
(n+2) A_{X} \stackrel{(x, y)}{\longrightarrow} A_{X}
$$

where the last summand is identified with $\mathcal{N}_{X / \mathbb{A}^{n+2}}$ via $\delta_{X}=[x y]^{*}$. Then $H^{1}\left(\mathcal{T}_{C}\right)$ and $H^{1}\left(\mathcal{T}_{X}\right)$ are both 1-dimensional and there is no higher cohomology, which means that the universal deformation of $C$ and $X$ are respectively given by

$$
u v-t, x y-s .
$$

Now consider the map

$$
f: C \rightarrow X, \quad(u, v) \mapsto\left(u^{m}, v^{m}, f_{1}^{u}+f_{1}^{v}, \ldots f_{n}^{u}+f_{n}^{v}\right)
$$

where the $f_{i}^{u}, f_{i}^{v}$ are unramified and without constant term. For example,

- if $n=0$, this is an $m$-fold branched cover on each component;

- if $n=1$, we may assume $f_{1}^{u}=u, f_{1}^{v}=v$ and then this map sends $C_{1}, C_{2}$ to the curves in $X_{1}, X_{2}$ with respective equations $x-z^{m}, y-z^{m}$, while $f(C)$ in total has equation $x+y-z^{m}$.

Then the Lie atom $f^{!} \mathcal{T}_{X}$ measures deformations of $(f, X)$ with $C$ fixed, together with a trivialization of the corresponding deformation of $X$. This atom can be written symbolically as

$$
(n+2) A_{C} \stackrel{\left(u^{m}, v^{m}\right)}{\longrightarrow} A_{C}
$$

in which it is convenient to identify $A_{C}$ as $F_{C, \mathbb{A}^{n+4,+}}[1]$, where $C \rightarrow \mathbb{A}_{u, v, x, y, z}^{n+4}=: P$ is the graph embedding, with equations

(3. 7.69)

$$
u v, x y, x-u^{m}, y-v^{m}, z_{i}-f_{i}^{u}-f_{i}^{v}, i=1, \ldots, n .
$$


Thus we may rewrite (3. 7.68) symbolically as

$$
n+2 F_{\dot{C}, P} \rightarrow n+2 A_{P} \oplus F_{\dot{C}, P} \rightarrow A_{P}
$$

where the last summand is generated by $\delta_{X}$. It is easy to check that

$$
\begin{array}{r}
H^{0}\left(f^{!} \mathcal{T}_{X}\right)=\left\{c_{x}(u) \partial_{x}+c_{y}(v) \partial_{y}+c_{\underline{z}}(u, v) \partial_{\underline{z}}:\right. \\
\left.c_{x}, c_{y}, c_{\underline{z}} \in A_{C}, c_{x}(0)=c_{y}(0)=0\right\}, \\
H^{1}\left(f^{!} \mathcal{T}_{X}\right)=\left(A_{C} /\left(u^{m}, v^{m}\right)\right) \cdot \epsilon_{X} .
\end{array}
$$

Moreover, the bracket (i.e. the obstruction) vanishes on $H^{0}\left(f^{!} \mathcal{T}_{f}\right)$. Thus, $\operatorname{Deff}\left(f^{!} \mathcal{T}_{X}\right)$ is a smooth, albeit infinite-dimensional space; in fact, it is quite elementary that $\operatorname{Deff}\left(f^{\prime} \mathcal{T}_{X}\right)$ can be identified with the space of pairs $\left(f_{u}, f_{v}\right)$ where $f_{u}, f_{v}$ are deformations, respectively, of the restrictions of $f$ to the $u$ and $v$-axes, considered as maps to $(x, \underline{z})$ (resp. $(y, \underline{z})$ )- space, that map the origin to the same point on the $z$-hyperplane; and as such, this space is clearly smooth.

Now consider the Lie atom $\mathcal{T}_{f}$, classifying general deformations of $f$. This can be written in the form

$$
\begin{array}{r}
A_{C}<\partial_{v}, \partial_{v}>\oplus A_{X}<\partial_{x}, \partial_{y}, \partial_{\underline{z}}>\oplus F_{\dot{C}, P}<\partial_{x}, \partial_{y}, \partial_{\underline{z}}> \\
\longrightarrow A_{C} \delta_{C} \oplus A_{X} \delta_{X} \oplus A_{P}<\partial_{x}, \partial_{y}, \partial_{\underline{z}}>\oplus F_{C, P} \delta_{X} \\
\left((u v)^{m-1}, \stackrel{\text { id } \left.,\left(u^{m}, v^{m}, 0\right), \epsilon\right)}{\longrightarrow} A_{P} \delta_{X}\right.
\end{array}
$$

where, wherever necessary, we replace $A_{C}$ by $F_{\dot{C}, P+}$ and $A_{X}$ by $F_{\dot{X}, Q+}$. Here each complex term, such as $F_{\dot{C}, P}$, should be viewed as extending leftward from the indicated position. Note that the last map reflects the fact that the differential of $\mathcal{T}_{f}$ maps $\delta_{C}=[u v]^{*}$ to

$$
d f\left(\delta_{C}\right)=(u v)^{m-1}[x y]^{*}=(u v)^{m-1} \delta_{X}
$$

As for brackets, note that

$$
\left[\delta_{C},[u v] \delta_{X}\right]=(u v)^{m-1} \delta_{X} \in A_{P} \delta_{X}=\mathcal{T}_{f}^{2} ;
$$

otherwise, the obvious generators of $\mathcal{T}_{f}^{1}$ have zero bracket. In particular we may, in computing the Jacobi-Bernoulli cohomology of $\mathcal{T}_{f}$, replace $\mathcal{T}_{f}^{2}$ by its quotient by the remaining generators of $F_{C}^{0}$ besides $u v$ (cf. (3. 7.69)), while eliminating the corresponding summands form $F_{C, P}^{0} \delta_{X}$.

Now consider a prospective special multiplicative cocycle $\epsilon=\epsilon(\phi, \psi)$ for $\mathcal{T}_{f}$, and write $\phi, \psi$ in the form

$$
\phi=\left(a \delta_{C}, b \delta_{X}\right) \in \mathcal{T}_{C}^{1} \oplus \mathcal{T}_{X}^{1}, \psi=\left(c_{x} \partial_{x}+c_{y} \partial_{y}, d[u v] \delta_{X}\right) \in \mathcal{T}_{X}^{0} \oplus F_{C, P}^{0} \otimes \mathcal{T}_{X}^{1} \subset\left(f^{!} \mathcal{T}_{X}\right)^{0},
$$


where $a, b$ are constants, $d=\sum_{i, j<m} d_{i, j} u^{i} v^{j}[u v]$, and by (3. 7.76) we may assume $d_{m-1, m-1}=0$. Now the Jacobi-Bernoulli cocycle condition on $\epsilon$ reads

$$
i(\phi)+d(\psi)+\sum_{i=1}^{\infty} B_{i} \operatorname{ad}(\psi)^{i}(\phi)=0
$$

where the $B_{i}$ are the Bernoulli numbers. Here all higher iterated adjoints (with $i>1$ ) vanish for reasons of degree. Thus (3. 7.78) yields

$$
a(u v)^{m-1}+b+c_{x} u^{m}+c_{y} v^{m}+d u v+B_{1} a d=0
$$

where, of course, $B_{1}$ is the 1 st Bernoulli number $B_{1}=-1 / 2$. Now, working modulo $\left(u^{m}, v^{m}\right)$ we see firstly that $d_{i, j}=0$ for $i \neq j$. Then, reading off coefficients of $(u v)^{i}$, we see that
(3. 7.80$)$
(3. 7.81$)$
(3. 7.82$)$
(3. 7.83$)$

$$
\begin{array}{r}
b=a d_{0} / 2 \\
d_{0}=a d_{1} / 2 \\
\ldots \quad d_{i}=a d_{i+1} / 2, i \leq m-3, \\
d_{m-2}=-a
\end{array}
$$

In particular,

(3. 7.84)

$$
b=-a^{m} / 2^{m-1} .
$$

Now consider the natural forgetful map

$$
\operatorname{Deff}(f) \rightarrow \operatorname{Deff}(C) \times \operatorname{Deff}(X)
$$

which, in terms of coordinates, is just given by projection to the $a b$ plane. Its fibre is just Deff $\left(f^{!} \mathcal{T}_{X}\right)$ which, as we have seen above, is smooth. Therefore this is is a smooth morphism onto the curve $D$ with equation (3. 7.84), whose fibre coincides with $\operatorname{Deff}\left(f^{!} \mathcal{T}_{X}\right)$. This curve $D$ in turn projects smoothly to the $a$-axis, viz. Deff $(C)$, but is ramified of degree $m$ over the $b$ axis, viz. $\operatorname{Deff}(X)$.

\section{THE PROJECTIVE CASE}

The purpose of this section is to adapt the construction of the tangent atom for an affinely embedded scheme $X \rightarrow P$, as given in the previous section, to the case of a projectively embedded scheme $X \rightarrow \mathbb{P}$. Whereas in the affine case the basic idea was to take for tangent atom $\mathcal{T}_{X}(P)$ the infinitesimal automorphisms of a resolution of $X$, the idea here, not surprisingly, is to construct the tangent atom $\mathcal{T}=\mathcal{T}_{X}(\mathbb{P})$ ) by looking instead at graded resolutions of the homogeneous ideal and homogeneous coordinate ring of $X$.

Unlike in the affine case, it is important here to work with the sheafified version $\tilde{\mathcal{T}}$. of the tangent atom, in addition to the graded module version $\mathcal{T}$. This has to do with the fact that free graded modules are not acyclic. Indeed when it comes to deformations, the non-acyclicity expresses itself in the difference between the 
appropriate cohomology of $\mathcal{T}$, which yields the projective deformations, while the corresponding (hyper-)cohomology of $\tilde{\mathcal{T}}$. yields all (abstract) deformations. In general, a scheme $X$ with $H^{2}\left(\mathcal{O}_{X}\right) \neq 0$ will admit nonprojective deformations, and the associated classes in the appropriate (Jacobi) cohomology of $\tilde{\mathcal{T}}_{X}$, considered as formal power series, will be necessarily non-convergent.

Although a tangent SELA $\mathcal{T}_{\bullet x}$, yielding all deformations, will be constructed in the next section in the generality of arbitrary algebraic schemes $X$, the sheafified tangent atom $\tilde{\mathcal{T}}_{\dot{X}}$ provides an adequate, and considerably simpler, substitute for $\mathcal{T}_{\bullet}$ in case $X$ is projective, as long as one restricts to infinitesimal deformations, avoiding questions of convergence.

4.1. Differential operators on projective spaces. Fix a projective space $\mathbb{P}=$ $\mathbb{P}(V)$ and let $S=S_{\mathbb{P}}=\operatorname{Sym}\left(V^{*}\right)$ be the corresponding homogeneous coordinate ring. We will generally identify a coherent sheaf $E$ on $\mathbb{P}$ with its 'de-sheafification', i.e. graded $S$-module

$$
\Gamma_{*}(E)=\bigoplus_{n \in \mathbb{Z}} \Gamma(E(n)) .
$$

We will also identify a graded $S$-module $M$ with a graded free resolution of $M$, as well as the sheafification $\tilde{M}$.

Let

$$
\hat{T}_{\mathbb{P}}=\mathfrak{D}^{1}\left(\mathcal{O}_{\mathbb{P}}(1), \mathcal{O}_{\mathbb{P}}(1)\right)
$$

be the Lie algebra of 1 st order differential endomorphisms of $\mathcal{O}_{\mathbb{P}}(1)$ (which we may identify with its module version, which is the shift $S[1])$. As is well known, we have a natural Lie isomorphism

$$
\hat{T}_{\mathbb{P}} \simeq \mathfrak{D}^{1}\left(\mathcal{O}_{\mathbb{P}}(m), \mathcal{O}_{\mathbb{P}}(m)\right)
$$

for any $m \neq 0$, and a sheaf isomorphism

$$
\hat{T}_{\mathbb{P}} \simeq V \otimes \mathcal{O}_{\mathbb{P}}(1) .
$$

The subsheaf

$$
E=\mathfrak{D}^{0}\left(\mathcal{O}_{\mathbb{P}}(1), \mathcal{O}_{\mathbb{P}}(1)\right) \simeq \mathcal{O}_{\mathbb{P}} \subset \hat{T}_{\mathbb{P}}
$$

is a Lie ideal and the Euler sequence

(4. 1.3)

$$
0 \rightarrow E \stackrel{e}{\rightarrow} \hat{T}_{\mathbb{P}} \rightarrow T_{\mathbb{P}} \rightarrow 0
$$

or in the module version

$$
0 \rightarrow S \rightarrow V \otimes S[1] \rightarrow \Gamma_{*} \hat{T}_{\mathbb{P}} \rightarrow 0
$$

is a sequence of Lie algebras and homomorphisms, where $E$ is an abelian ideal and the action of $T_{\mathbb{P}}$ on $E=\mathcal{O}_{\mathbb{P}}$ is the standard one. There is a natural action pairing called the standard action

$$
\hat{T}_{\mathbb{P}} \times \mathcal{O}_{\mathbb{P}}(m) \rightarrow \mathcal{O}_{\mathbb{P}}(m) .
$$


For $m \neq 0$ this is clear from the identification (4. 1.2), while for $m=0$ it comes via (4. 1.3) from the action of $T_{\mathbb{P}}$ on $\mathcal{O}_{\mathbb{P}}$. For $m=0$, this action is a derivation in the usual sense. For all $\mathrm{m}$, it is a 'derivation relative to the action on $\mathcal{O}_{\mathbb{P}}$ ', in the sense that

$$
v(a f)=a v(f)+v(a) f, v \in \hat{T}_{\mathbb{P}}, a \in \mathcal{O}_{\mathbb{P}}, f \in \mathcal{O}_{\mathbb{P}}(m) .
$$

From (4. 1.2) we deduce, via post-multiplication, isomorphisms

$$
\hat{T}_{\mathbb{P}}(k) \simeq \mathfrak{D}^{1}\left(\mathcal{O}_{\mathbb{P}}(m), \mathcal{O}_{\mathbb{P}}(m+k)\right), \forall m, k .
$$

This again defines the standard action

$$
\hat{T}_{\mathbb{P}}(k) \times \mathcal{O}_{\mathbb{P}}(m) \rightarrow \mathcal{O}_{\mathbb{P}}(m+k) .
$$

We note that over each standard affine $P=D_{X_{i}} \subset \mathbb{P}$, the identification $\mathcal{O}_{P} \stackrel{X_{i}}{\rightarrow}$ $\left.\mathcal{O}(1)\right|_{P}$ induces a Lie-theoretic splitting of (4. 1.3), i.e.

$$
\left.\hat{T}_{\mathbb{P}}\right|_{P} \simeq E \oplus T_{P} .
$$

Moreover, the $E$ summand (i.e. the Euler operator) acts trivially on $\left.\mathcal{O}(m)\right|_{P}$ for all $m$.

4.2. Construction. Fix a closed subscheme $X \subset \mathbb{P}=\mathbb{P}(V)$, and consider a free resolution of its ideal sheaf $\mathcal{I}=\mathcal{I}_{X}$ :

$$
\ldots \rightarrow \mathcal{F}^{i}=\bigoplus \mathcal{F}_{\alpha}^{i} \rightarrow \ldots \rightarrow \mathcal{F}^{0} \rightarrow \mathcal{I}
$$

where we may assume

$$
\mathcal{F}_{\alpha}^{i}=\mathcal{O}_{\mathbb{P}}\left(-m_{i \alpha}\right), m_{i \alpha}>0, \forall i .
$$

One way to obtain some (though not all) sheaf resolutions (4. 2.6) is to start with a graded resolution $F$. of the graded ideal $I^{\cdot}=I_{X}$ and sheafify. Resolutions obtained in this manner are said to be full. A general sheaf resolution (4. 2.6) is obtained by sheafifying a complex of free modules which, at each stage, is exact in sufficiently high degrees, i.e. whose cohomology is supported on the irrelevant prime of $S$.

As before, we augment $\mathcal{F}$. to a resolution $\mathcal{F}_{+}$of $\mathcal{O}_{X}$ by setting $\mathcal{F}_{+}^{1}=\mathcal{O}_{\mathbb{P}}$. For each $\alpha, \beta$, we denote by

$$
\delta_{\alpha \beta}^{i} \in \operatorname{Hom}\left(\mathcal{F}_{\alpha}^{i}, \mathcal{F}_{\beta}^{i+1}\right)=\mathcal{O}_{\mathbb{P}}\left(m_{i \alpha}-m_{i+1, \beta}\right)
$$

the appropriate component of the differential of the resolution.

Then we may define the (sheafy) normal atom as

$$
\tilde{\mathcal{N}}=\tilde{\mathcal{N}}_{X / \mathbb{P}}=\operatorname{Hom} \cdot\left(\mathcal{F}, \mathcal{F}_{+}\right) .
$$

This is a sheaf of Lie atoms (in fact, dgla's) on $\mathbb{P}$. The restriction of $\tilde{\mathcal{N}}_{X / \mathbb{P}}$ on a standard affine open $P \subset \mathbb{P}$ is just the sheafified version of the affine normal atom $\tilde{\mathcal{N}}_{X \cap P / P}$. On the other hand, applying $\Gamma_{*}$ we get the global graded version over $S_{\mathbb{P}}$, denoted $\mathcal{N}_{X / \mathbb{P}}$. 
Now the idea, as in the affine case, is to construct the sheafy tangent atom $\tilde{\mathcal{T}}$. as the mapping cone of a suitable map

$$
T_{\mathbb{P}} \otimes \mathcal{F}_{+} \rightarrow \tilde{\mathcal{N}}_{X / \mathbb{P}}
$$

where $T_{\mathbb{P}}$ itself is identified with a mapping cone as in the Euler sequence (4. 1.3). The global graded case is analogous.

Now the terms of $\tilde{\mathcal{T}}$. are defined as follows

$$
\begin{aligned}
\tilde{\mathcal{T}}^{i} & =\left(\hat{T}_{P} \otimes \mathcal{F}_{+}^{i+1}\right) \oplus\left(E \otimes \mathcal{F}_{+}^{i+2}\right) \oplus \mathfrak{g l}^{i}(\mathcal{I}), \quad i \leq 0, \\
& =\mathfrak{g l}^{i}(\mathcal{I}) \oplus \operatorname{Hom}\left(\mathcal{F}^{-i+1}, \mathcal{O}_{\mathbb{P}}\right), \quad i \geq 1 .
\end{aligned}
$$

where, as before, we identify

$$
\mathfrak{g l}^{i}(\mathcal{I})=\mathfrak{g l}^{i}(\mathcal{F} \cdot)=\bigoplus_{j} \operatorname{Hom}\left(\mathcal{F}^{j}, \mathcal{F}^{i+j}\right) .
$$

So, schematically,

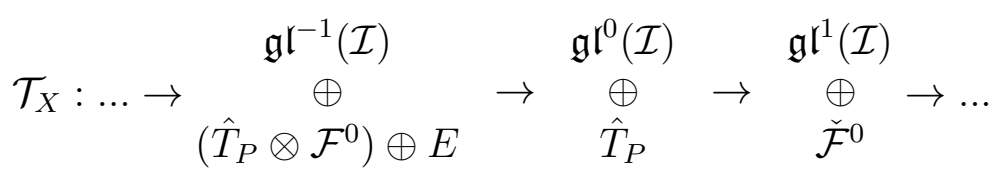

(with the middle term in degree 0 ). As in the affine case, $\tilde{\mathcal{T}}$. will be constructed so as to admit $\tilde{\mathcal{N}}$ as subcomplex and $T_{\mathbb{P}} \otimes \mathcal{F}_{+}$as quotient complex, thus the only components of the differential that require definition are those going from $T_{\mathbb{P}} \otimes \mathcal{F}_{+}$ to $\tilde{\mathcal{N}}$.

Those components are defined as follows.

- For $i=0$, the map $\hat{T}_{\mathbb{P}} \rightarrow \tilde{\mathcal{N}}^{1}$ is

$$
v \mapsto \bigoplus_{j, \alpha, \beta} v\left(\delta_{\alpha \beta}^{j}\right)
$$

(cf. (4. 1.2) and (4. 2.7))

- For $i<0$, the map $\hat{T}_{\mathbb{P}} \otimes \mathcal{F}_{\gamma}^{i+1} \rightarrow \tilde{\mathcal{N}}^{i+1}$ is

$$
v \otimes a \mapsto \bigoplus_{j, \alpha, \beta} \mu\left(a, v\left(\delta_{\alpha \beta}^{j}\right)\right)
$$

where $\mu$ (abusively) denotes the composite map

$$
\mathcal{F}^{k} \otimes \operatorname{Hom}\left(\mathcal{F}^{r}, \mathcal{F}^{s}\right) \rightarrow \operatorname{Hom}\left(\mathcal{F}^{r}, \mathcal{F}^{k} \otimes \mathcal{F}^{s}\right) \stackrel{\mu}{\rightarrow} \operatorname{Hom}\left(\mathcal{F}^{r}, \mathcal{F}^{k+s-1}\right)
$$

where the latter $\mu$ is the map induced by the multiplication on $\mathcal{O}_{X}$ as in $\S 3$.

- form $i=-1$, the map $E \rightarrow \tilde{\mathcal{N}}^{0}$ is

(4. 2.14)

$$
1 \mapsto \bigoplus_{j, \alpha} m_{j \alpha} \mathrm{id}_{\mathcal{F}_{\alpha}^{j}}
$$


- For $i<-1$, the map $E \otimes \mathcal{F}^{i+2} \rightarrow \tilde{\mathcal{N}}^{i+1}$ is

$$
1 \otimes a \mapsto \bigoplus_{j, \alpha} m_{i \alpha} \mu\left(a, \mathbf{i d}_{\mathcal{F}_{\alpha}^{j}}\right) .
$$

All these formulas are dictated, on the one hand, by the natural action of $\hat{T}_{\mathbb{P}}$ on the various $\mathcal{F}_{\alpha \beta}^{i}$, and on the other hand, by the concept of defining the differential going into

$\tilde{\mathcal{N}} \subset \operatorname{Hom}\left(\mathcal{F}_{+}, \mathcal{F}_{+}\right)$via commutator with the differential of $\mathcal{F}_{+}$, as in (3. 1.11) and (3. 1.13). Also, via the splitting (4. 1.5), they are compatible with the definition of the affine tangent atom $\mathcal{T}_{X \cap P}(P)$ for each standard affine $P=D_{X_{i}} \subset \mathbb{P}$.

Next we must show that $\tilde{\mathcal{T}}$ is a complex, i.e. that $d^{2}=0$. On all terms except those involving $E$, the proof is similar to the affine case, based on the fact that $\hat{T}_{\mathbb{P}}$ acts as derivations. Alternatively, one can argue based on (3. 1.11) and (3. 1.13). On the terms involving $E$, i.e. $E \otimes \mathcal{F}^{i}$, the required vanishing is an immediate consequence of Euler's identity, i.e. the fact that $E$ acts as $m$.id on $\mathcal{O}(m)$.

Thus we have defined $\tilde{\mathcal{T}}=\tilde{\mathcal{T}}_{X}(\mathbb{P})$ as a complex of sheaves (of graded free $\mathcal{O}_{\mathbb{P}}$ modules) on $\mathbb{P}$. We note that the restriction of $\tilde{\mathcal{T}}$ on a standard affine open $P=$ $D_{X_{i}} \subset \mathbb{P}$ is quasi-isomorphic to the sheafy version of the tangent atom $\tilde{\mathcal{T}}_{X \cap P}(P)$. Note also that by construction, there is a subcomplex $\tilde{\mathcal{T}}_{X / \mathbb{P}}$ of $\tilde{\mathcal{T}}$ (omitting all the $T_{\mathbb{P}} \otimes \mathcal{F}$. terms), such that $\tilde{\mathcal{T}}$ is the mapping cone of a map $T_{\mathbb{P}} \otimes \mathcal{F} \rightarrow \tilde{\mathcal{T}}_{X / P}$.

Next, we define an action of $\tilde{\mathcal{T}}_{X / \mathbb{P}}$ on the complex $\mathcal{F} \cdot(m) \sim \mathcal{I}(m), \forall m$, analogous to the affine case. We let $\tilde{\mathcal{N}}$ act in the obvious way, as in the affine case. We let $\hat{T}_{\mathbb{P}}$ act in the standard way on each $\mathcal{O}(k)$ summand of $\mathcal{F}^{i}(m)$ and similarly for $E$ (which acts on $\mathcal{O}(k)$ as multiplication by $-k$ ). We note that the restriction of this action on a standard affine $P \subset \mathbb{P}$ is compatible with the action of $\mathcal{T}_{X / P}$ on $F$. defined previously, via the splitting (4. 1.5) with $E$ acting trivially on $\mathcal{O}_{P}$.

Next, the action of $\tilde{\mathcal{T}}_{X / \mathbb{P}}$ on $\mathcal{F}$. extends to an action of $\tilde{\mathcal{T}}_{X / \mathbb{P}}$ on $\mathcal{F}_{+}(m)$, where $\tilde{\mathcal{N}}$ acts trivially on $\mathcal{F}_{+}^{1}(m)=\mathcal{O}_{\mathbb{P}}(m)$. Similarly, there is an action of $\tilde{\mathcal{T}}_{X / P}$ on $\tilde{\mathcal{N}}$, where $\tilde{\mathcal{N}}$ acts on itself via bracket (adjoint action), and $\hat{T}_{\mathbb{P}}$ and $E$ act on each line bundle summand $\mathcal{O}(k) \subset \tilde{\mathcal{N}}$ in the standard way.

Next, the action of $\tilde{\mathcal{T}}_{X / \mathbb{P}}$ on $\mathcal{F}_{+}(m)$ extends to an action of $\tilde{\mathcal{T}}$ on $\mathcal{F}_{+}(m)$ via the standard recipe

$$
\langle a \otimes v, b\rangle=\mu(a,\langle v, b\rangle), a \in \mathcal{F}^{i}, b \in \mathcal{F}_{+}^{j}, v \in \hat{T}_{\mathbb{P}}
$$

where $\langle v, b\rangle$ refers to the pairing defined previously as part of the action of $\mathcal{T}_{X / \mathbb{P}}$

Next, we define a bracket on $\tilde{\mathcal{T}}$ and $\tilde{\mathcal{T}}_{X / \mathbb{P}}$ : this is defined as the usual bracket (signed commutator) on $\mathcal{N}$ and the standard bracket on $T_{\mathbb{P}}$ (which is compatible with the bracket on $\hat{T}_{\mathbb{P}}$ ). As for mixed terms, we define

$$
[v, a]=\langle v, a\rangle, \quad v \in \hat{T}_{50}, a \in \tilde{\mathcal{N}}
$$


where again $\langle v, a\rangle$ refers to the pairing defined previously. This suffices to define a bracket on $\tilde{\mathcal{T}}_{X / \mathbb{P}}$. We extend this to a bracket on $\tilde{\mathcal{T}}$ by setting

$$
[a \otimes v, b]=\langle a \otimes v, b\rangle
$$

cf. (4. 2.16) As before, the necessary axioms (e.g. Jacobi identity, action rule etc.) can either be easily verified directly or deduced from the corresponding properties on the affine pieces $X \cap P \subset P$.

This completes the construction of the tangent dgla $\tilde{\mathcal{T}}_{X}(\mathbb{P})$ for any projective scheme $X \subset \mathbb{P}$. As in the affine case, we can show that

(i) the dgla quasi-isomorphism class of $\tilde{\mathcal{T}}_{X}(\mathbb{P})$ depends only on the subscheme $X \subset \mathbb{P}$ and not on the resolution and other choices;

(ii) there is a reduced version $\tilde{\mathcal{T}}_{X}^{\text {red }}(\mathbb{P})$, again independent of the resolution;

(iii) the weak equivalence classes of $\tilde{\mathcal{T}}_{X}(\mathbb{P})$ and $\tilde{\mathcal{T}}_{X}^{\text {red }}(\mathbb{P})$ are independent of the embedding $X \rightarrow \mathbb{P}$ and depend on the isomorphism class of $X$ only.

Remark 4.1. If $X$ is as above (closed in $\mathbb{P}$ ) and $X^{\prime} \subset X$ is any open subset, the restriction $\left.\tilde{\mathcal{T}}_{X}(\mathbb{P})\right|_{X^{\prime}}$ yields a dgla sheaf on $X^{\prime}$ that will be called it tangent dgla and denoted $\tilde{\mathcal{T}}_{X^{\prime}}(\mathbb{P})$. This extends the notion of tangent dgal to the case of an arbitrary quasi-projective scheme.

\section{Algebraic schemes: Tangent SEla}

The purpose of this section is to extend the notion of tangent object (dgla or atom) of affine schemes and their maps to the case of general (algebraic) schemes. This extension will take the form of a semi-simplicial Lie algebra or SELA. The reason for this added level of complexity is that, given an affine covering $\left(X_{\rho}\right)$ of a scheme, each tangent dgla $\mathcal{T}_{X_{\rho}}$ is in reality defined with respect to some particular affine embedding $X_{\rho} \rightarrow P_{\rho}$, and these embeddings are not mutually related. Consequently, the $\mathcal{T}_{X_{\rho}}$ need not glue together to a dgla, because the restrictions of $\mathcal{T}_{X_{\rho}}$ and $\mathcal{T}_{X_{\sigma}}$ on $X_{\rho} \cap X_{\sigma}$ are only weakly equivalent (and indirectly so at that), and this relation is too weak for ordinary gluing. Nonetheless the relation of weak equivalence is strong enough to yield a SELA .

5.1. Construction. Here we construct the tangent SELA of a separated algebraic scheme $X$ over $\mathbb{C}$ (the separatedness does not seem to be essential, but is convenient). To fix ideas we focus on the non-sheafy version, though the (coherently) sheafified version can be constructed in the same way. Let $\left(X_{\rho}\right)$ be an affine open covering of $X$ indexed by a well-ordered set, and for each $\rho$ let $P_{\rho}$ be an affine space with a closed embedding

(5. 1.1)

$$
\iota_{\rho}: X_{\rho} \subset P_{\rho} .
$$

Set $B_{\rho}=A_{P_{\rho}}$. We call the system $\left(X_{\rho} \subset P_{\rho}\right)$ an affine embedding system for $X$. Note that via

$$
X_{\rho} \cap X_{\sigma}=\Delta_{X} \cap \underset{51}{X_{\rho}} \times X_{\sigma} \subset X \times X,
$$


$X_{\rho} \cap X_{\sigma}$ embeds as a closed subscheme of $X_{\rho} \times X_{\sigma}$, hence of $P_{\rho} \times P_{\sigma}$. Similarly, for any multi-index $\rho_{0}<\ldots<\rho_{k}$, we define

$$
X_{\left(\rho_{0}, \ldots, \rho_{k}\right)}=X_{\rho_{0}} \cap \ldots \cap X_{\rho_{k}}, \quad P_{\left(\rho_{0}, \ldots, \rho_{k}\right)}=P_{\rho_{0}} \times \ldots \times P_{\rho_{k}}
$$

and the natural closed embedding

$$
\iota_{\left(\rho_{0}, \ldots, \rho_{k}\right)}: X_{\left(\rho_{0}, \ldots, \rho_{k}\right)} \subset P_{\left(\rho_{0}, \ldots, \rho_{k}\right)},
$$

and we denote the ideal of the latter by $I_{\left(\rho_{0}, \ldots, \rho_{k}\right)}$. We call the system

$$
\left(X_{\left(\rho_{0}, \ldots, \rho_{k}\right)} \subset P_{\left(\rho_{0}, \ldots, \rho_{k}\right)},\left(\rho_{0}<\ldots<\rho_{k}\right), k \geq 0\right)
$$

the simplicial extension of the affine embedding system $\left(X_{\rho} \subset P_{\rho}\right)$. Note that the defining equations for the image of $\iota_{\left(\rho_{0}, \ldots, \rho_{k}\right)}$ consist of defining equations for the images of individual embeddings $\iota_{\rho_{i}}$, together with equations for the small diagonal on $X^{k+1}$. The latter are of course generated by the pullbacks of the equations of the small diagonal in $X^{k}$ via the various coordinate projections $X^{k+1} \rightarrow X^{k}$. Therefore, it is possible to choose mutually compatible free resolutions for all the $I_{\left(\rho_{0}, \ldots, \rho_{k}\right)}$, and we denote these by $F_{\left(\rho_{0}, \ldots, \rho_{k}\right)}$. In fact, we may assume that

$$
F_{\left(\rho_{0}, \ldots, \rho_{k}\right)}^{1}=B_{\left(\rho_{0}, \ldots, \rho_{k}\right)}:=\bigotimes_{0}^{k} B_{\rho_{i}}
$$

$$
F_{\left(\rho_{0}, \ldots, \rho_{k}\right)}^{i}=\bigoplus_{j=0}^{k}\left(F_{\rho_{j}}^{i} \otimes B_{\left(\rho_{0}, \ldots, \rho_{k}\right)}\right) \oplus \Delta_{\left(\rho_{0}, \ldots, \rho_{k}\right)}^{i}, i \leq 0,
$$

where $\Delta_{\left(\rho_{0}, \ldots, \rho_{k}\right)}$ is a lifting to $B_{\left(\rho_{0}, \ldots, \rho_{k}\right)}$ of a free resolution of the small diagonal $X_{\left(\rho_{0}, \ldots, \rho_{k}\right)} \subset \prod_{j=0}^{k} X_{\rho_{j}}$ and moreover for any biplex

$$
\rho^{k}=\left(\rho_{0}, . ., \rho_{k}\right) \subset \rho^{k+1}=\left(\rho_{0}, \ldots, \rho_{k+1}\right),
$$

if we let

$$
\pi_{\rho^{k+1}, \rho^{k}}: P_{\rho^{k+1}} \rightarrow P_{\rho^{k}}
$$

denote the natural projection, then we have a direct summand inclusion

$$
\pi_{\rho^{k+1}, \rho^{k}}^{*} F_{\rho^{k}}:=\pi_{\rho^{k+1}, \rho^{k}}^{-1} F_{\rho^{k}} \otimes B_{\rho^{k+1}} \rightarrow F_{\rho^{k+1}}
$$

Putting together these groups and maps, and twisting by the appropriate sign, i.e. $\epsilon\left(\rho^{k}, \rho^{k+1}\right)$, we get an 'extrinsic Čech (double) complex'

(5. 1.8)

$$
\mathrm{XC}\left(\mathcal{O}_{X}\right): \bigoplus_{\rho^{0}} F_{+\rho^{0}} \rightarrow \ldots \rightarrow \bigoplus_{\rho^{k}} F_{+\rho^{k}} \rightarrow \ldots
$$


A similar construction can be applied to any coherent sheaf on $X$. Actually this complex is quasi-isomorphic to the usual Cech complex of $\mathcal{O}_{X}$, but we can do more with it. Note that the map (5. 1.7) gives rise to a direct summand inclusion

$$
\pi_{\rho^{k+1}, \rho^{k}}^{*}\left(\mathfrak{g l} \cdot\left(I_{\rho^{k}}\right)\right) \rightarrow \mathfrak{g l} \cdot\left(I_{\rho^{k+1}}\right),
$$

whence a dgla map

$$
\delta_{\rho^{k}, \rho^{k+1}}: \mathcal{N}_{X_{\rho^{k}} / P_{\rho^{k}}} \rightarrow \mathcal{N}_{X_{\rho^{k+1}} / P_{\rho^{k+1}}}
$$

Then we can similarly construct a 'normal SELA'

$$
\mathcal{N}_{X_{\bullet} / P_{\bullet}}: \ldots \rightarrow \bigoplus_{\rho^{k}} \mathcal{N}_{X_{\rho^{k}} / P_{\rho^{k}}} \rightarrow \ldots
$$

Likewise, we have an 'ambient tangent complex' $T_{P_{\bullet}}$ and $T_{P_{\bullet}} \otimes \mathrm{XC}\left(\mathcal{O}_{X}\right)$ and a map

$$
T_{P_{\bullet}} \otimes \mathrm{XC}\left(\mathcal{O}_{X}\right) \rightarrow \mathcal{N}_{X_{\bullet} / P_{\bullet}}
$$

Finally, we define the tangent SELA of $X$ (with reference to the simplicial system $\left.\left(X_{\bullet}, P_{\bullet}\right)\right)$ to be the mapping cone of this, and denote it by $\mathcal{T}_{X_{\bullet}}\left(X_{\bullet}, P_{\bullet}\right)$ or simply $\mathcal{T}_{X}$. This is the SELA whose value on the simplex $\rho^{k}$ is the dgla $\mathcal{T}_{X_{\rho^{k}}}\left(P_{\rho^{k}}\right)$. By Theorem 1.3, there is an associated Jacobi-Bernoulli complex $J \cdot\left(\mathcal{T}_{X}.\right)$, which we denote by $J_{X}$ and refer to as the Jacobi-Bernoulli complex of $X$. Up to filtered, comultiplicative weak equivalence, it depends only on the isomorphism class of $X$ as scheme over $\mathbb{C}$. Therefore the deformation ring of $X$

$$
R_{X}=\mathbb{C} \oplus \mathbb{H}^{0}\left(J_{X}\right)^{*}
$$

is canonically defined. In the next section we relate $R_{X}$ to flat deformations of $X$ over artin rings. For any artin local $\mathbb{C}$-algebra $S$, we set

$$
J_{X, S}=J \cdot\left(\mathcal{T}_{X \bullet} \otimes \mathfrak{m}_{S}\right)
$$

and note that via the natural map $J_{\dot{X}, S} \rightarrow J_{\dot{X}} \otimes \mathfrak{m}_{S}$, any class $\epsilon \in \mathbb{H}^{0}\left(J_{\dot{X}, S}\right)$ yields a local homomorphism ('classifying map')

$$
{ }^{t} \epsilon: R_{X} \rightarrow S \text {. }
$$

As in the affine case, this construction may be extended from the case of schemes to that of maps. Thus let

$$
f: X \rightarrow Y
$$

be a morphism of schemes. Then we can choose respective affine coverings

$$
X_{\alpha} \rightarrow P_{\alpha}, Y_{\alpha} \rightarrow Q_{\alpha} \text { such that } f\left(X_{\alpha}\right) \subset Y_{\alpha} .
$$

Then for each simplex $\rho^{k}$, the restriction of $f$ yields a morphism

$$
f_{\rho^{k}}: X_{\rho^{k}} \rightarrow Y_{\rho^{k}},
$$


and for this we have an associated tangent dgla $\mathcal{T}_{f_{\rho^{k}}}$. Putting these together, we get a tangent SELA (with respect to the given affine coverings)

$$
\mathcal{T}_{f}: \ldots \rightarrow \mathcal{T}_{f_{\rho^{k}}} \rightarrow \ldots
$$

As before, $\mathcal{T}_{f}$ can be described as a mapping cone:

$$
\mathcal{T}_{f}=\operatorname{cone}\left(\mathcal{T}_{X} \bullet \mathcal{T}_{Y \bullet} \rightarrow f^{!} \mathcal{T}_{Y} \bullet .\right.
$$

Thus we have SELA morphisms

$$
\mathcal{T}_{f} \rightarrow \mathcal{T}_{Y}, \mathcal{T}_{f} \rightarrow \mathcal{T}_{X}, f^{!} \mathcal{T}_{Y}[1] \rightarrow \mathcal{T}_{f} .
$$

Correspondingly, we have a Jacobi-Bernoulli complex $J_{f}$, a deformation ring $R_{f}$ together with maps $R_{X} \rightarrow R_{f}, R_{Y} \rightarrow R_{f}$.

Remark 5.1. When $X$ is smooth, its tangent SELA is equivalent to a dgla, e.g. the Kodaira-Spencer algebra, a soft dgla resolution of the tangent sheaf.

Remark 5.2. When $X$ is quasi-projective, i.e. a locally closed subscheme of a projective space $\mathbb{P}$ with closure $\bar{X}$, we have constructed in the previous section a tangent dgla sheaf $\tilde{\mathcal{T}}_{\bar{X}}(\mathbb{P})$

5.2. Basic properties. We summarize the basic properties of the tangent SELA $\mathcal{T}_{\mathcal{X}_{\bullet}}\left(\mathcal{X}_{\bullet}, P_{\bullet}\right)$ as follows.

Theorem 5.3. $\quad$ (i) $\mathcal{T}_{\mathcal{X}_{\bullet}}\left(\mathcal{X}_{\bullet}, P_{\bullet}\right)$ is a SELA and $\mathrm{XC}\left(\mathcal{O}_{X}\right)$-module and acts on $\mathrm{XC}\left(\mathcal{O}_{X}\right)$.

(ii) The weak equivalence class $\mathcal{T}_{X_{\bullet}}$ of $\mathcal{T}_{\mathcal{X}_{\bullet}}\left(\mathcal{X}_{\bullet}, P_{\bullet}\right)$ depends only on $X$ and is functorial.

(iii) If $X$ is smooth, $\mathcal{T}_{X}$. is weakly equivalent to the (Čech complex of) the usual tangent algebra $T_{X}$.

Proof. (i) The fact that $\mathcal{T}_{\mathcal{X}_{\bullet}}\left(\mathcal{X}_{\bullet}, P_{\bullet}\right)$ is a SELA is clear from the construction, and the actions by and on $\mathrm{XC}\left(\mathcal{O}_{X}\right)$ are also clear component by component.

(ii) Any two affine embedding systems $\left(X_{\bullet}, P_{\bullet}\right),\left(X_{\bullet}, Q_{\bullet}\right)$ are dominated by a third, say $\left(X_{\bullet}, R_{\bullet}\right)$, and we get maps

$$
\mathcal{T}_{X_{\bullet}}\left(X_{\bullet}, P_{\bullet}\right) \rightarrow \mathcal{T}_{X}\left(X_{\bullet}, R_{\bullet}\right) \leftarrow \mathcal{T}_{X_{\bullet}}\left(X_{\bullet}, Q_{\bullet}\right) .
$$

These maps are termwise weak equivalence hance, by a standard spectral sequence argument, overall weak equivalences as well. This proves the independence of $\mathcal{T}_{X_{\bullet}}\left(X_{\bullet}, P_{\bullet}\right)$ on the affine embedding system used to define it. The functoriality is proved similarly.

(iii) This follows from Corollary 3.4 above, since in he smooth case we may take for each $\mathcal{T}_{X_{\rho}}$ the usual tangent algebra, and these glue together properly on overlaps. 


\section{DEFoRMATIONS OF SCHEMES}

6.1. Classification. Deformations of an algebraic scheme $X / \mathbb{C}$ can be classified in terms of the associated tangent SELA $\mathcal{T}_{X}$ and its Jacobi-Bernoulli cohomology. Consider first the case of an affine scheme $X \subset P$ (notations as in $\S 3$ ). Let $S$ be a local artinian $\mathbb{C}$-algebra. Then a flat deformation of $X$ over $S$ is determined by, and determines, up to certain choices, an element

$$
\phi \in \mathcal{T}_{X}^{1}(P) \otimes \mathfrak{m}_{S}=\operatorname{Hom}^{1}\left(F_{\dot{X}}, F_{+X}\right) \otimes \mathfrak{m}_{S}
$$

known as a Kodaira-Spencer cochain, which satisfies the integrability condition

$$
\partial \phi=-\frac{1}{2}[\phi, \phi] .
$$

The deformation corresponding to $\phi$ can be determined e.g. as the subscheme of $P \times \operatorname{Spec}(S)$ having $\left(F_{\dot{X}} \otimes S, \partial+\phi\right)$ as resolution; we may denote this by $X^{\phi}$.

Now globally, let $X$ be an algebraic scheme over $\mathbb{C}$ and as in $\S 5$ choose an affine embedding system

$$
\iota_{\rho}: X_{\rho} \rightarrow P_{\rho} .
$$

This gives rise as in $\S 5$ to a representative for the tangent SELA $\mathcal{T}_{X}$. Now suppose given a deformation $\mathfrak{X}$ of $X$ over $S$ as above. This restricts for each $\rho$ to a deformation $\mathfrak{X}_{\rho}$ of $X_{\rho}$, whence a Kodaira-Spencer cochain

$$
\phi_{\rho} \in \mathcal{T}_{X_{\rho}}^{1}\left(P_{\rho}\right) \subset \mathcal{T}_{X}^{1}\left(P_{\bullet}\right),
$$

satisfying an integrability condition as in (6. 1.2), so that the restricted deformation $\mathfrak{X}_{\rho}$ is $S$-isomorphic to $X_{\rho}^{\phi_{\rho}}$. Moreover, the fact that $\phi_{\rho}$ and $\phi_{\sigma}$ restrict to equivalent deformations of $X_{\rho \sigma} \subset P_{\rho \sigma}$ yields an $S$ - isomorphism

$$
X_{\rho}^{\phi_{\rho}} \cap X_{\sigma} \simeq X_{\sigma}^{\phi_{\sigma}} \cap X_{\rho} ;
$$

both of these are closed subschemes of $P_{\rho \sigma} \times \operatorname{Spec}(S)$ and the isomorphism (6. 1.3) extends to an $S$ - automorphism of $P_{\rho \sigma} \times \operatorname{Spec}(S)$, necessarily of the form

$$
\exp \left(t_{\rho \sigma}\right), t_{\rho \sigma} \in T_{P_{\rho \sigma}} \otimes \mathfrak{m}_{S} .
$$

Then we get two resolutions of $X_{\rho}^{\phi_{\rho}} \cap X_{\sigma}$, the 'original' one with differential $\partial+\phi_{\rho}$, and the one pulled back from $X_{\sigma}^{\phi_{\sigma}} \cap X_{\rho}$, whose differential is $\partial+\phi_{\sigma}$. It is easy to see and well known that the two resolutions differ by an isomorphism of the form $\exp \left(u_{\rho \sigma}\right)$ where $u_{\rho \sigma} \in \mathfrak{g l}^{0}\left(F_{\dot{X}_{\rho}}\right) \otimes \mathfrak{m}_{S}$. Thus all in all there is a (uniquely determined) element

$$
\psi_{\rho \sigma} \in \mathcal{T}_{X_{\rho \sigma}}^{0} \otimes \mathfrak{m}_{S}=\left(T_{P_{\rho \sigma}} \oplus \mathfrak{g l}^{0}\left(F_{X_{\rho \sigma}}\right)\right) \otimes \mathfrak{m}_{S}
$$

such that

$$
\exp \left(\psi_{\rho \sigma}\right)\left(\partial+\phi_{\sigma}\right) \exp \left(-\psi_{\rho \sigma}\right)=\partial+\phi_{\rho} .
$$

More explicitly, the latter equation reads

$$
\partial \exp \left(\psi_{\rho \sigma}\right)=-\phi_{\rho} \exp _{55}\left(\psi_{\rho \sigma}\right)+\exp \left(\psi_{\rho \sigma}\right) \phi_{\sigma} .
$$


Multiplying by $\exp \left(-\psi_{\rho \sigma}\right)$, we get

$$
D\left(-\operatorname{ad}\left(\psi_{\rho \sigma}\right)\right)(\partial \psi)=-\exp \left(-\psi_{\rho \sigma}\right) \phi_{\rho} \exp \left(\psi_{\rho \sigma}\right)+\phi_{\sigma}
$$

where $D(x)$ is as in (1. 2.6), with inverse $C(x)$ the generating function for the Bernoulli numbers. Multiplying the latter equation by $C\left(-\operatorname{ad}\left(\psi_{\rho \sigma}\right)\right)$ and using the simple identity $C(-x) e^{-x}=C(x)$, we conclude

$$
\partial \psi_{\rho \sigma}=-C\left(\operatorname{ad}\left(\psi_{\rho \sigma}\right)\right) \phi_{\rho}+C\left(-\operatorname{ad}\left(\psi_{\rho \sigma}\right)\right) \phi_{\sigma} .
$$

The latter equation (6. 1.4) is just the condition that the component in bidegree $(0,1)$ of the coboundary $\partial \epsilon\left(\phi_{\bullet}, \psi_{\bullet}\right)$ should vanish. Also, by construction, we clearly have

(6. 1.5)

$$
\exp \left(\psi_{\rho \sigma}\right) \exp \left(\psi_{\sigma \tau}\right)=\exp \left(\psi_{\rho \tau}\right)
$$

Thus, putting $(6.1 .2,6.1 .4,6.1 .5)$ together and using the derivation property of the differentials in the Jacobi-Bernoulli complex, $\epsilon\left(\phi_{\bullet}, \psi_{\bullet}\right)$ is a special multiplicative cocycle in the Jacobi-Bernoulli complex $J\left(\mathcal{T}_{X}\right) \otimes \mathfrak{m}_{S}$. Conversely, given a special multiplicative cocycle $\epsilon\left(\phi_{\bullet}, \psi_{\bullet}\right)$ with values in $S$, the $\phi_{\bullet}$ data yields a collection of deformations of the affine pieces of $X$, while the $\psi_{\bullet}$ glues these deformations together. These processes are inverse to each other up to an automorphism, and are precise mutual inverses when there are no automorphisms. Hence

Theorem 6.1. Let $X$ be an algebraic scheme over $\mathbb{C}$ such that $H^{0}\left(\mathcal{T}_{X}\right)=0$. Then for any local artin $\mathbb{C}$-algebra $S$, there is a natural bijection between the set of equivalence classes of flat deformations of $X$ over $S$ and the set of local homomorphisms from $R_{X}$ to $S$.

6.2. Obstructions. Let $S$ be a local artin algebra, $I<S$ an ideal contained in the socle $\operatorname{ann}_{S}\left(\mathfrak{m}_{S}\right)$ and $\bar{S}=S / I$. Let $\bar{\epsilon}=\epsilon\left(\bar{\phi}_{\bullet}, \bar{\psi}_{\bullet}\right)$ be a special multiplicative cocycle with coefficients in $\bar{S}$. Let $\phi_{\bullet}, \psi_{\bullet}$ be arbitrary liftings of $\phi_{\bullet}, \psi_{\bullet}$ with coefficients in $S$. Thus, $\epsilon=\epsilon\left(\phi_{\bullet}, \psi_{\bullet}\right)$ is not necessarily a cocycle. However, it is easy to check that the coboundary $\partial \epsilon$ lies in

$$
\left(F_{1} J_{X}\right)^{1} \otimes I=\left(K^{0}\left(\mathcal{T}_{X}\right)^{2} \oplus K^{1}\left(\mathcal{T}_{X}\right)^{1} \oplus K^{2}\left(\mathcal{T}_{X}\right)^{0}\right) \otimes I
$$

(this is because it dies modulo $I$ ) and moreover, that $\partial \epsilon$ is a cocycle for tot $\left(K \cdot\left(\mathcal{T}_{X}\right) \cdot\right) \otimes$ $I$ (the latter because it is a cocycle for $J_{X, S}$ ). Thus, we obtain a cohomology class

$$
\operatorname{ob}\left(\bar{\phi}_{\bullet}, \bar{\psi}_{\bullet}\right) \in H^{2}\left(\mathcal{T}_{X}\right) \otimes I=H^{2}\left(\operatorname{tot}\left(K^{\cdot}\left(\mathcal{T}_{X}\right)^{\cdot}\right) \otimes I .\right.
$$

This class is independent of choices and represents the obstruction to lifting $\bar{\epsilon}$ to a special multiplicative cocycle with coefficients in $S$.

Example 6.2. Let $X$ be a quadric cone in $P=\mathbb{P}^{3}$, with equation $q=x_{1} x_{2}-x_{3}^{2}$. Let $f: L \rightarrow X$ be the inclusion of the line with equations $x_{1}=x_{2}=x_{3}$. Then $\mathcal{T}_{X}$ is as in Example 3.1 and $f^{!} \mathcal{T}_{X}[-1]$ is the Lie atom (special case of SELA )

$$
I_{L} \mathcal{T}_{X 6} \rightarrow \mathcal{T}_{X}
$$


This atom, being the mapping cone of $\mathcal{T}_{f} \rightarrow \mathcal{T}_{X} \oplus \mathcal{T}_{Y}$, classifies deformations of the map $f$ together with trivializations of the corresponding deformations of $X$ and $Y$. Also, as $L$ is smooth we may identify $\mathcal{T}_{L}$ with the utual tangent algebra $T_{L}$, and there is a natural map

$$
\mathcal{T}_{L} \rightarrow f^{!} \mathcal{T}_{X}
$$

whose associated Lie atom, i.e. mapping cone, is $\mathcal{N}_{L / X}$, which classifies deformations of $L$ in $X$. Then it is well known that

$$
T_{P} \otimes \mathcal{O}_{L} \simeq T_{L} \oplus 2 \mathcal{O}_{L}(1), N_{L / P} \simeq 2 \mathcal{O}_{L}(1) .
$$

The map $T_{P} \otimes \mathcal{O}_{L} \rightarrow \mathcal{O}_{L}(2)$ vanishes on $T_{L}$ and has cokernel a skyscaper $\mathbb{C}$ at the vertex of the cone. The kernel of the induces map $N_{L / P} \rightarrow \mathcal{O}_{L}(2)$ is generated ny $x_{0} v, x_{1} v$ where

$$
v=\partial / \partial x_{1}-\partial / \partial x_{2} \in T_{P}(-1) \subset \mathcal{T}_{X}^{0}(-1) .
$$

Thus

$$
\mathbb{H}^{0}\left(\mathcal{N}_{L / X}\right) \simeq\left\{a_{0} x_{0} v+a_{1} x_{1} v: a_{0} . a_{1} \in \mathbb{C}\right\} \simeq \mathbb{C}^{2}, \mathbb{H}^{1}\left(\mathcal{N}_{L / X}\right)=\left[x_{0}^{2}\right] \simeq \mathbb{C}
$$

Set

$$
g=x_{0}\left(x_{2}-x_{1}\right) \in I_{L}(2)=I_{L} \mathcal{T}_{X}^{1} .
$$

Then the fact that $g=x_{0} v(f)$ shows that the element $\epsilon=\epsilon\left(x_{0} v, g\right)$ is a special multiplicative cocycle for $f^{!} \mathcal{T}_{X}$ with coefficients in $\mathbb{C}=\mathbb{C}[t] /(t)$. The obstruction to lifting $\epsilon$ over $S=\mathbb{C}[t] /\left(t^{2}\right)$ is precisely

$$
x_{0} v(g)=-2 x_{0}^{2} \neq 0 .
$$

Therefore $\epsilon$ does not lift. In fact, since the first obstruction map goes onto $\mathbb{H}^{1}\left(\mathcal{N}_{L / X}\right)$, there are no higher obstructions. Therefore in dual coordinates, the formal deformation ring is

$$
\hat{R}=\hat{R}\left(\mathcal{N}_{L / X}\right) \simeq \mathbb{C}\left[\left[a_{0}^{*}, a_{1}^{*}\right]\right] /\left(\left(a_{0}^{*}\right)^{2}\right),
$$

i.e. a double 'line'; the universal deformation over $\hat{R}$ is given by $\epsilon\left(a_{0}^{*} g, a_{0}^{*} x_{0} v+a_{1}^{*} x_{1} v\right)$. Since set-theoretically the locus of lines in $X$ is a conic, this shows the conic occurs with multiplicity 2.

A more involved calculation that we won't do in detail actually yields the equations of this double conic in a standard $\mathbb{A}^{4}$ neighborhood on $L$ in the Grassmannian of lines in $\mathbb{P}^{3}$, which is coordinatized via the vector fields

$$
x_{0} v, x_{1} v, x_{0} w, x_{1} w, w:=\partial / \partial x_{1}-\partial / \partial x_{3} .
$$


6.3. Applications: relative obstructions, stable subschemes and surjections. Let $f: X \rightarrow Y$ be an embedding of a closed subscheme. We consider the question of 'relative obstructions' i.e. obstructions to lifting a given deformation of $Y$ to a deformation of $f$. These are obstructions for the mapping cone of $\mathcal{T}_{f} \rightarrow \mathcal{T}_{Y}$, which is the same as that of $\mathcal{T}_{X} \rightarrow f^{!} \mathcal{T}_{Y}$ (cf. (5. 1.10)). Locally, if $Y \rightarrow P$ is an affine embedding, such obstructions have values in $\operatorname{Ext}^{1}\left(I_{X / Y}, \mathcal{O}_{X}\right)$, where $I_{X / Y}=I_{X / P} / I_{Y / P}$ is independent of $P$ even as complex up to quasi-isomorphism (not just weak equivalence). Hence, global obstructions also have values in $\operatorname{Ext}^{1}\left(I_{X / Y}, \mathcal{O}_{X}\right)$. In reasonably good cases though, the obstruction group can be narrowed considerably. The following result sharpens Thm. 1.1 of [9]

Theorem 6.3. Let $X \subset Y$ be a closed subscheme having no component contained in the singular locus of $Y$. Then obstructions to deforming $X \rightarrow Y$ relative to deforming $Y$ are in

$$
\operatorname{Im}\left(\operatorname{Ext}_{Y}^{1}\left(I_{X / Y} / I_{X / Y}^{2}, \mathcal{O}_{X}\right) \rightarrow \operatorname{Ext}_{Y}^{1}\left(I_{X / Y}, \mathcal{O}_{X}\right)\right) .
$$

Corollary 6.4. Assumptions as in Theorem 6.3, assume additionally that $X \rightarrow Y$ is a regular embedding with normal bundle $N$ and $H^{1}(X, N)=0$. Then $X \rightarrow Y$ is 'relatively unobstructed' or 'stable' relative to $Y$, in the sense that it deforms with every deformation of $Y$; furthermore the Hilbert scheme of $Y$ is smooth of dimension $h^{0}(X, N)$ at the point corresponding to $X$.

This Corollary generalizes a result of Kodaira [4] in the smooth case.

To sketch the proof of Thorem 6.3, first working locally, let $F_{\dot{Y}}$ be a free resolution of $I_{Y / P}$ and extend it to a free resolution $F_{X}$ of $I_{X / P}$, such that, termwise,

$$
F_{X}^{i}=F_{Y}^{i} \oplus F_{X / Y}^{i}
$$

where $F_{X / Y}^{i}$ is a suitable free complement, $F_{\dot{Y}} \rightarrow F_{\dot{X}}$ is a map of complexes (though not a direct summand inclusion), and $F_{\dot{X} / Y} \otimes \mathcal{O}_{Y}$, which is a quotient complex of $F_{\dot{X}}$, is a free resolution of $I_{X / Y}$. We may also assume that $F_{X / Y}$ contains a subcomplex $F_{X^{2}}$ (with termwise direct summands) resolving $I_{X}^{2}$. A deformation of $Y$ yields a linear map

$$
v: F_{Y}^{0} \rightarrow \mathcal{O}_{X} .
$$

The obstruction to lifting this to a deformation of $X$ is given by

$$
v \circ \delta: F_{X / Y}^{-1} \rightarrow \mathcal{O}_{X}
$$

where $\delta: F_{X / Y}^{-1} \rightarrow F_{Y}^{0}$ is the 'connecting map' from the resolution. $\delta$ takes a relation among generators of $I_{X} \bmod I_{Y}$ to the appropriate linear combination of generators of $I_{Y}$. Now, and this is the point, our assumption about singularities means that no generator of $I_{Y}$ can be in $I_{X}^{2}$, or more precisely, that

$$
I_{X}^{2} \cap I_{Y} \subset I_{X} I_{Y}
$$


This implies that $\delta\left(F_{X^{2}}^{-1}\right) \subset I_{X} F_{Y}^{0}$. Since $v$ is $\mathcal{O}_{P}$-linear, it follows that the composition $v \circ \delta$ is zero on $F_{X^{2}}^{-1}$ and lives in the image as in (6. 3.7). This proves the result in the affine case, and the extension to the global case is straightforward.

If $Y$ is a projective locally complete intersection, the reduced tangent algebra ${ }^{\operatorname{red}} \mathcal{T}_{Y}$ has an important numerical property related to the dualizing sheaf of $Y$. Namely, viewing ${ }^{\operatorname{red}} \mathcal{T}_{Y}$ as an element in the Grothendieck group $K(Y)$ of vector bundles (or coherent sheaves), it has a well-defined determinant line bundle, and we have

$$
\operatorname{det}\left({ }^{\operatorname{red}} \mathcal{T}_{Y}\right)=\omega_{Y}^{-1}=\omega_{P}^{-1} \operatorname{det}\left(N_{Y / P}\right)
$$

where $P$ is an ambient projective space. Indeed this follows immediately from the construction of the dualizing sheaf and the discussion of Serre duality theory in [1]. This, together with the fundamental dimension inequality, Corollary 1.5, can be applied to study deformations of maps of curves to varieties:

Theorem 6.5. Let $f: X \rightarrow Y$ be a map of a smooth projective curve of genus $g$ to a projective locally complete intersection variety of dimension $n$ such that $f(X)$ is not contained in the singular locus of $Y$. Then the space Deff $(f)$ of deformations of $f$ fixing $X, Y$ is of dimension at least $\chi=X . \omega_{Y}^{-1}+n(1-g)$; moreover, $L$ if $\chi>3, g=$ $0,>1, g=1$, or $>0, g>1, f$ admits deformations so that $f(X)$ moves.

Proof. In our case $f^{!} \operatorname{red} \mathcal{T}_{Y}$ is a finite complex of vector bundles on $X$, acyclicl in degrees $\neq 0,1$. By Riemann-Roch, we have

$$
\chi\left(f^{! \operatorname{red}} \mathcal{T}_{Y}\right)=\left(h^{0}-h^{1}\right)\left(f^{! \operatorname{red}} \mathcal{T}_{Y}\right)=X . \omega_{Y}^{-1}+n(1-g) .
$$

Therefore by Corollary 1.5, our conclusion follows.

Next we consider an application to surjections is (compare [9], Thm 2.1):

Theorem 6.6. Let $f: X \rightarrow Y$ be a projective morphism with

$$
f_{*}\left(\mathcal{O}_{X}\right)=\mathcal{O}_{Y}, R^{1} f_{*}\left(\mathcal{O}_{X}\right)=0 .
$$

Then $f$ deforms with every deformation of $X$.

Proof. Here we use the fact that the mapping cone of $\mathcal{T}_{f} \rightarrow \mathcal{T}_{X}$ is equivalent to that of $\mathcal{T}_{Y} \rightarrow f^{!} \mathcal{T}_{Y}$. To prove the result it suffices to show the following

${ }^{*}$ ) the natural map $\mathcal{T}_{Y} \rightarrow f^{!} \mathcal{T}_{Y}$ induces a surjection on $H^{1}$ and an injection on $H^{2}$.

Indeed the $H^{1}$-surjectivity property implies that any first-order deformation of $X$ lifts to a deformation of $f$; then the $H^{2}$-injectivity property allows us to extend this inductively, via obstruction theory ( $\$ 3.2)$, to $n$th order deformations. To prove $(*)$, note that $f^{!} \mathcal{T}_{Y}$ can be represented by the tensor product $\mathcal{T}_{Y} \otimes \mathrm{XC}\left(\mathcal{O}_{X}\right)$, which is a double complex with terms $\mathcal{T}_{Y}^{i} \otimes \mathrm{XC}^{j}\left(\mathcal{O}_{X}\right)$. Then the spectral sequence of a double complex (or an elementary substitute) yields our conclusion. 
Morphisms $f$ satisfying the hypotheses of the Theorem occur in diverse situations, e.g. regular fibre spaces and resolutions of rational singularities. The Theorem says that those schemes which admit a structure such as $f$ form an open neighborhood of $X$ in Moduli.

Theorem 6.7. Let $f: X \rightarrow Y$ be a proper surjective morphism étale in codimension 1 where $X$ is normal. Then any deformation of $f$ is determined by the associated deformation of $Y$.

Proof. It will suffice to prove that the mapping cone $\mathcal{T}_{X} \rightarrow f^{!} \mathcal{T}_{Y}$ is exact in degrees $\leq 1$. Working locally, we may view $X$ as a subscheme of $Y \times R, R$ an affine space, and consider a free resolution $J$ of the ideal of $X$ in $Y \times R$. Let $K$ be the kernel of the natural map $\mathcal{H o m}\left(J^{0}, \mathcal{O}_{X}\right) \rightarrow \mathcal{H o m}\left(J^{-1}, \mathcal{O}_{X}\right)$. Then $K$ is a torsion-free $\mathcal{O}_{X}$ module and the natural map $k: T_{R} \otimes \mathcal{O}_{X} \rightarrow K$ is an isomorphism in codimension 1 by our assumption that $f$ is étale in codimension 1 . As $X$ is normal, this easily implies $k$ is an isomorphism, which proves our assertion.

Corollary 6.8. A small resolution of a singularity $Y$ is (infinitesimally) rigid relative to $Y$; hence the set of small resolutions of a given $Y$ is at most countable.

Smallness is of course an essential hypothesis here. One wonders, however, whether 'countable' may be replaced by 'finite' in the conclusion.

Under stronger hypotheses on the size of the exceptional locus, we can actually identify the deformations of $f$ and $Y$ (compare [9], Thm. 3.5):

Theorem 6.9. Let $f: X \rightarrow Y$ be a proper surjective morphism étale in codimension 2 with $X$ locally Cohen-Macaulay everywhere and lci in codimension 2. Then any deformation of $Y$ lifts to a deformation of $f$.

Proof. We work as in the proof of Theorem 3.6. As there, consider the complexes

$$
\begin{aligned}
& K_{Y}: \ldots \mathcal{F}_{1, Y}^{-1} \otimes \mathcal{O}_{Y} \rightarrow F_{Y}^{0} \otimes \mathcal{O}_{Y} \rightarrow \Omega_{Q} \otimes \mathcal{O}_{Y}, \\
& K_{X}: \ldots \mathcal{F}_{1, Y}^{-1} \otimes \mathcal{O}_{X} \rightarrow F_{X}^{0} \otimes \mathcal{O}_{X} \rightarrow \Omega_{P} \otimes \mathcal{O}_{X},
\end{aligned}
$$

as well as the complex on $X$ :

$$
f^{!} K_{Y}: \ldots \mathcal{F}_{1, Y}^{-1} \otimes \mathcal{O}_{X} \rightarrow F_{Y}^{0} \otimes \mathcal{O}_{X} \rightarrow \Omega_{Q} \otimes \mathcal{O}_{X}
$$

As in the aforementioned proof, we have

$$
\operatorname{Hom} \cdot\left(K_{Y}, \mathcal{O}_{Y}\right) \simeq \mathcal{T}_{Y}, \operatorname{Hom} \cdot\left(K_{X}, \mathcal{O}_{X}\right) \simeq \mathcal{T}_{X} \text { Hom }\left(f^{!} K_{Y}, \mathcal{O}_{X}\right) \simeq f^{\prime} \mathcal{T}_{Y} .
$$

Now let $K_{f}$ be the mapping cone of the functorial map $f^{!} K_{Y} \rightarrow K_{X}$ (cf. subsubsection 3.2.7; this cone is a complex in degrees $\leq 1$, and its cohomology is supported on the non-étale locus of $f$, which has codimension $\geq 3$ in $X$. Thefore it follows from Ischebeck's theorem as in the proof of Theorem 3.6 that we have

$$
\operatorname{Ext}^{i}\left(K_{f}, \mathcal{O}_{X}\right)=0, i \leq 2 .
$$


From this is follows that the natural map

$$
H^{i}\left(\mathcal{T}_{X}\right) \rightarrow H^{i}\left(f^{!} \mathcal{T}_{Y}\right)
$$

is bijective for $i \leq 1$ and injective for $i=2$; in other words,

( $\star$ the map $\mathcal{T}_{X} \rightarrow f^{!} \mathcal{T}_{Y}$ is a weak equivalence.

From this last statement the Theorem follows by a purely formal, standard argument that runs as follows. Given an artinian local algebra $S$ and a local homomorphism

$$
\phi_{Y}: R\left(\mathcal{T}_{Y}\right) \rightarrow S,
$$

we see by using $(\star)$ and artinian induction that the induced map $f^{!} \phi_{Y}: R\left(f^{!} \mathcal{T}_{Y}\right) \rightarrow$ $S$ lifts to a map $\phi_{X}: R\left(\mathcal{T}_{X}\right) \rightarrow S$, and this means precisely that $\phi_{Y}$ lifts to a deformation $\left(\phi_{X}, \phi_{Y}\right)$ of $f$.

Remark 6.10. The Theorem does not extend to morphisms with a codimension-2 exceptional locus; for example, it fails for a small resolution of a 3-fold ODP.

\section{REFERENCES}

1. R. Hartshorne, Algebraic Geometry, Springer, Berlin, 1977.

2. F. Ischebeck, Eine dualität zwischen den funktoren Ext und Tor, J. Alg. 11 (1969), 510-531.

3. J.Harris and D. Mumford, The kodaira dimension of the moduli space of curves, Inventiones math. (1984).

4. K. Kodaira, A theorem of completeness of characteristic systems for analytic families of compact submanifolds of complex mainifolds., Ann. Math. 75 (1962), 146-162.

5. _ Complex manifolds and deformations of complex structures, Springer, Berlin, 1986.

6. S. Lichtenbaum and M. Sclessinger, The cotangent complex of a morphism., Trans. AMS 128 (1967), 41-70.

7. H. Matsumura, Commutative algebra 2e, Benjamin/Cummings, Reading, Mass, 1980.

8. Z. Ran, Enumerative geometry of families of singular plane curves, Invent. math 97 (1989), 447.

9. _ Stability of certain holomorphic maps, J. Diff. Geom. 34 (1991), 37-47.

10. _ Canonical infinitesimal deformations, J. Algebraic Geometry 9 (2000), 43.

11. Lie atoms and their deformation theory, Geometric and Functional Analysis 18 (2008), 184-221.

12. E. Sernesi, Deformations of algebraic schemes, Springer International, 2006.

13. V.S. Varadarajan, Lie groups, Lie algebras and their representations, Springer, Berlin, 1973.

UNIVERSITY OF CALIFORNIA, RIVERSIDE

E-mail address: ziv.ran@ucr.edu 